University of Tennessee Health Science Center UTHSC Digital Commons

$11-1999$

\title{
Portal Imaging Using a CSI (TL) Scintillator Coupled to a Cooled CCD Camera
}

Amit Sawant

University of Tennessee Health Science Center

Follow this and additional works at: https://dc.uthsc.edu/dissertations

Part of the Equipment and Supplies Commons

\section{Recommended Citation}

Sawant, Amit , "Portal Imaging Using a CSI (TL) Scintillator Coupled to a Cooled CCD Camera" (1999). Theses and Dissertations (ETD). Paper 234. http://dx.doi.org/10.21007/etd.cghs.1999.0277.

This Thesis is brought to you for free and open access by the College of Graduate Health Sciences at UTHSC Digital Commons. It has been accepted for inclusion in Theses and Dissertations (ETD) by an authorized administrator of UTHSC Digital Commons. For more information, please contact jwelch30@uthsc.edu. 


\title{
Portal Imaging Using a CSI (TL) Scintillator Coupled to a Cooled CCD Camera
}

\author{
Abstract \\ The purpose of this research was to design a high performance digital portal imaging \\ system, using a transparent $x$-ray scintillator coupled to a cooled CCD camera. Theoretical \\ analysis using Monte Carlo simulation was performed to calculate the QDE, SNR and DQE of \\ the system. A prototype electronic portal imaging device (EPID) was built, using a $12.7 \mathrm{~mm}$ \\ thick, $20.32 \mathrm{~cm}$ diameter, CsI (TI) scintillator, coupled to an Astromed ® liquid nitrogen cooled \\ CCD TV camera. The system geometry of the prototype EPID was optimized to achieve high \\ spatial resolution. Experimental evaluation of the prototype EPID was performed, by \\ determining its spatial resolution, contrast resolution, depth of focus and light scatter. Images of \\ phantoms, animals and human subjects were acquired using the prototype EPID and were \\ compared with those obtained using conventional and high contrast portal film and a commercial \\ EPID. An image processing protocol was developed. The protocol was comprised of \\ preprocessing, noise removal and image enhancement algorithms. An adaptive median filter \\ algorithm for the removal of impulse noise was developed, analyzed and incorporated into the \\ image processing protocol.
}

Results from the theoretical analysis and experimental evaluation have indicated that the performance of the CsI (TI) - CCD system is comparable or superior to that of current commercial and experimental portal imaging technologies, such as high contrast portal film, commercial TV camera based EPIDs, and amorphous silicon based flat panel EPIDs.

\section{Document Type}

Thesis

\section{Degree Name}

Master of Science (MS)

\section{Program}

Biomedical Engineering

Research Advisor

Herbert D. Zeman

\section{Keywords}

digital portal imaging, treatment verification, megavoltage $\mathrm{x}$-ray imaging, transparent scintillator, CCD camera

\section{Subject Categories}

Analytical, Diagnostic and Therapeutic Techniques and Equipment | Equipment and Supplies | Medicine and Health Sciences 


\title{
Development of a Transparent Scintillator Based Digital Portal Imaging System
}

\author{
A Thesis \\ Presented for \\ The Graduate Studies Council \\ The University of Tennessee, Memphis \\ In Partial Fulfillment \\ Of the Requirements for the Degree \\ Master of Science \\ From the University of Tennessee
}

By

Amit Sawant

December 1999 
Chapter 8 @ by SPIE - The International Society for Optical Engineering.

All other material (C) by Amit Sawant, 1999

All rights reserved 


\section{DEDICATION}

This thesis is dedicated to Sri Ranjit Maharaj, my spiritual guide and to my parents, Ramakant Sawant and Anvita Sawant 


\section{ACKNOWLEDGEMENTS}

I would like to thank my major professor, Dr. Herbert Zeman for his guidance and advice for the past two years. I would also like to thank my other committee members, Dr. Frank DiBianca, Dr. Sanjiv Samant and Dr. Milan Buncick for their suggestions and comments regarding my work. And finally, I would like to thank my parents, who encouraged me to pursue higher education and supported me through the highs and lows in my career. 


\begin{abstract}
The purpose of this research was to design a high performance digital portal imaging system, using a transparent $\mathrm{x}$-ray scintillator coupled to a cooled CCD camera. Theoretical analysis using Monte Carlo simulation was performed to calculate the QDE, SNR and DQE of the system. A prototype electronic portal imaging device (EPID) was built, using a $12.7 \mathrm{~mm}$ thick, $20.32 \mathrm{~cm}$ diameter, CsI (Tl) scintillator, coupled to an Astromed ${ }^{\circledR}$ liquid nitrogen cooled CCD TV camera. The system geometry of the prototype EPID was optimized to achieve high spatial resolution. Experimental evaluation of the prototype EPID was performed, by determining its spatial resolution, contrast resolution, depth of focus and light scatter. Images of phantoms, animals and human subjects were acquired using the prototype EPID and were compared with those obtained using conventional and high contrast portal film and a commercial EPID. An image processing protocol was developed. The protocol was comprised of preprocessing, noise removal and image enhancement algorithms. An adaptive median filter algorithm for the removal of impulse noise was developed, analyzed and incorporated into the image processing protocol.
\end{abstract}

Results from the theoretical analysis and experimental evaluation have indicated that the performance of the CsI (Tl) - CCD system is comparable or superior to that of current commercial and experimental portal imaging technologies, such as high contrast portal film, commercial TV camera based EPIDs, and amorphous silicon based flat panel EPIDs. 


\section{Table of Contents}

Chapter 1. Portal Imaging - Background and Significance 10

Radiotherapy in Cancer Treatment 10

Portal Imaging 11

$\begin{array}{lr}\text { Limitations of Megavoltage X-ray Imaging } & 12\end{array}$

Portal Imaging Modalities 14

Motivation for Use of a Transparent Scintillator 21

Chapter 2. Basic Physics and Electronics 23

Interaction of High Energy X-rays with Matter 23

Scintillation Detector Principles 28

CCD Based Imaging Systems $\quad 32$

$\begin{array}{lr}\text { Chapter 3. Theoretical Analysis } & 42\end{array}$

Introduction $\quad 42$

Background $\quad 44$

Methods $\quad 46$

Results $\quad 51$

Conclusions $\quad 56$

$\begin{array}{ll}\text { Chapter 4. System Design } & 58\end{array}$

Basic Design $\quad 58$

Design Considerations $\quad 59$

Noise: Sources and Removal $\quad 65$

$\begin{array}{lr}\text { Chapter 5. Experimental Evaluation } & 68\end{array}$

Bases for Experimental Evaluation $\quad 68$

$\begin{array}{ll}\text { Spatial Resolution } & 68\end{array}$

$\begin{array}{ll}\text { Depth of Field } & 71\end{array}$

$\begin{array}{ll}\text { Contrast Resolution } & 73\end{array}$

$\begin{array}{ll}\text { Light Scatter in CsI (Tl) Crystal } & 74\end{array}$

$\begin{array}{ll}\text { Mirror Glare } & 77\end{array}$

$\begin{array}{lr}\text { Chapter 6. Subject Imaging } & 80\end{array}$

Imaging Objects $\quad 80$

$\begin{array}{lr}\text { Chest Phantom } & 80\end{array}$

$\begin{array}{lr}\text { Animal Imaging } & 84\end{array}$

$\begin{array}{ll}\text { Human Subjects } & 85\end{array}$ 
$\begin{array}{ll}\text { Chapter 7. Image Processing } & 90\end{array}$

Image Processing Protocol $\quad 90$

$\begin{array}{ll}\text { Pre-processing } & 90\end{array}$

Noise Removal (Impulse Noise) 91

Image Enhancement $\quad 92$

$\begin{array}{ll}\text { Chapter 8. Adaptive Median Filter Algorithm } & 97\end{array}$

Introduction $\quad 97$

Development of the Adaptive Median Filter Algorithm 98

Processing Medical Images $\quad 110$

$\begin{array}{ll}\text { Conclusion } & 117\end{array}$

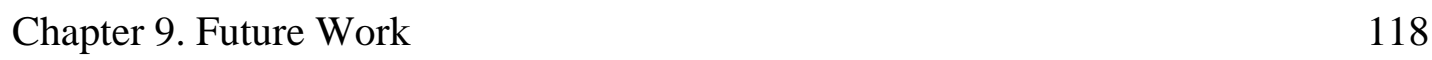

Optimal Thickness of CsI (Tl) and Leaded Glass 118

$\begin{array}{ll}\text { Shielding } & 119\end{array}$

Development of Proposed Full Field of View EPID 119

Analysis of Noise Power Spectrum 120

$\begin{array}{ll}\text { Clinical Evaluation } & 121\end{array}$

$\begin{array}{ll}\text { Chapter 10. Conclusion } & 122\end{array}$

$\begin{array}{ll}\text { References } & 124\end{array}$

$\begin{array}{ll}\text { Vita } & 129\end{array}$ 


\section{List of Figures}

Figure 2-1 Compton collision between a photon of energy hv and an electron assumed to be stationary

Figure 2-2 Energy band structure and scintillation mechanism of an activated crystalline

$\begin{array}{ll}\text { scintillator } & 30\end{array}$

Figure 2-3 Movement of charge packets by clocking the CCD electrodes 34

Figure 2-4 Basic structure and operation of a CCD imaging array and CCD readout 38

Figure 3-1 Experimental arrangement for the CCD-CsI (Tl) based portal imaging system 45

Figure 3-2 Absorbed energy distribution for $12.7 \mathrm{~mm} \mathrm{CsI} \mathrm{(Tl)} \mathrm{with} 1.5 \mathrm{~mm}$ lead radiator 52

Figure 3-3 Theoretical QDE and DQE calculations for 28 thickness combinations of CsI (Tl) and lead equivalent of leaded glass 53

Figure 3-4 QDE and DQE of a $12.7 \mathrm{~mm} \mathrm{CsI} \mathrm{(Tl)} \mathrm{crystal} \mathrm{with} 1.5 \mathrm{~mm}$ lead radiator plotted against the thickness of water absorber $\quad 54$

Figure 3-5 Comparison of DQE(f) of the proposed CsI (Tl) - CCD system, a TV camera based EPID and an amorphous silicon flat panel EPID $\quad 55$

Figure 4-1 Experimental arrangement for the CCD-CsI (Tl) based portal imaging system 58

$\begin{array}{lr}\text { Figure 4-2 CCD - CsI (Tl) portal imager assembly } & 59\end{array}$

Figure 4-3 Depth of field for a photographic lens 62

$\begin{array}{ll}\text { Figure 5-1 Bar pattern and modulation plot } & 69\end{array}$

Figure 5-2 Modulation transfer function for CsI (Tl) - CCD EPID 70

Figure 5-3 Variation in MTF with distance between lens and CsI (Tl) screen 72

Figure 5-4. CCD-CsI (Tl) images of low contrast phantom, $5 \mathrm{~cm}$ thick Lucite with $10 \mathrm{~mm}$ diameter holes 2, 3, 4, 4.5, 5, and $6 \mathrm{~mm}$ deep, and $2 \mathrm{~mm}$ diameter holes 2, 3, 4, 4.5, 4.5 and 5 mm deep 73

Figure 5-5 Comparison of contrast resolution $\quad 74$ 
Figure 5-6 Absorption of scattered light inside the CsI (Tl) crystal

Figure 5-7. Surface plots of light scatter glare for six positions of a highly collimated $6 \mathrm{MvP}$ x$\begin{array}{ll}\text { ray beam } & 76\end{array}$

Figure 5-8 Mechanism of mirror glare in phosphor screen based EPIDs $\quad 78$

Figure 5-9 Surface plots of mirror glare in the CsI (Tl) screen $\quad 79$

Figure 6-1 Images of a chest phantom $\quad 81$

Figure 6-2 Comparison of the CsI (Tl) - CCD system with ECL film and Philips SRI - 10083

Figure 6-3 Composite image of the leg of a pig constructed from six views 85

Figure 6-4 Lateral pelvic images: Comparison with portal film 87

Figure 6-5 Lateral images of the pelvic region at $10 \mathrm{MV}$, using the CCD-CsI(Tl) EPID 89

Figure 8-1 Outputs (d), (e), (f) of a $5 \times 5$ median filter with different input signals i.e. (a) constant neighborhood, (b) impulse and (c) fine structure 99

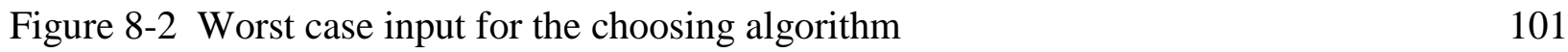

Figure 8-3 Comparison between the ordinary median filter and the adaptive median filter using $\begin{array}{ll}\text { an 8-bit grayscale test image } & 108\end{array}$

Figure 8-4 Histogram of the difference between the uncorrupted image and the median filtered image

Figure 8-5 Dual energy X-ray images of a preserved rat's foot 111

Figure 8-6 High resolution CT image of a slice of the head of a hamster 113

Figure 8-7 Portal images of a pig's foot taken at $6 \mathrm{MV}, 18 \mathrm{MU} \quad 116$

Figure 8-8 Ultrasound images taken with a 3.5 MHz phased array transducer 116 


\section{CHAPTER 1}

\section{Portal Imaging - Background and Significance}

\subsection{Radiotherapy in Cancer Treatment}

Radiotherapy is extensively used as a primary or secondary treatment for many kinds of cancer such as Hodgkin's disease, certain head and neck tumors, and tumors of the oral cavity, pharynx, cervix, prostate, uterus and lung. ${ }^{1}$ Radiotherapy uses high energy radiation such as megavoltage $\mathrm{x}$-rays, generated using a linear accelerator, gamma rays, generated by a radioactive source such as Cobalt - 60, or high energy electron beams to destroy or damage cancer cells. Radiation treatment is administered by directing a precisely defined beam of high energy radiation to the tumor area. Cancer cells grow and divide more rapidly than normal cells and hence are more susceptible to radiation. The goal of radiation treatment is to destroy cancerous tissue, while causing minimal damage to the surrounding normal tissue.

\subsubsection{Treatment Planning}

A multistep treatment planning procedure is performed before beginning the actual therapy. ${ }^{2}$ The first step of treatment planning consists of precisely defining the target volume in three dimensions. The site of the tumor is identified using multiple CT or MRI slices. The critical normal structures that lie within the potential treatment volume are identified and the treatment area and the planned dose are adjusted so as to deliver minimal radiation to these critical structures. A detailed treatment plan is developed using the diagnostic imaging data and isodose distributions for the high energy beam. The treatment volume may be targeted from multiple 
directions to maximize the effect of radiation on the tumor, while minimizing damage to normal tissue.

\subsubsection{Accurate Dose Delivery}

After the treatment plan is finalized for a given volume, the treatment area in each direction is identified by taking diagnostic radiographs through each of the treatment portals. These diagnostic images are taken using a simulator, which is a diagnostic x-ray unit, identical in geometry to the treatment unit. The patient is immobilized in the treatment position and the treatment fields are outlined on the skin using a suitable semi permanent marker. The corresponding area is also marked on the simulator films. Custom made collimating blocks of a low melting point, high-density alloy are constructed. These blocks are used during the treatment to ensure that the area surrounding the treatment region does not receive a significant amount of radiation.

\subsection{Portal Imaging}

\subsubsection{Verification}

An important aspect of radiation treatment is the verification of the localization of dose delivery to the clinical treatment volume (CTV). This verification is performed either by a dosimetric estimation or a geometric localization verification. Dosimetric verification is performed by measuring the dose at some points inside or outside the patient and comparing the measured

values with theoretically calculated dose values at the same points. ${ }^{3}$ Verification of geometric localization is performed by imaging the patient before or during the treatment and comparing 
these images with simulation images. The acquisition of megavoltage $\mathrm{x}$-ray images through the treatment portal of the therapy unit is known as portal imaging.

\subsubsection{Motivation for Portal Imaging}

Accurate patient positioning and beam placement are essential for effective targeting of the CTV. It has been clinically proven that high tumor control rates along with minimal damage to normal

tissue can be achieved with increased accuracy in dose delivery. ${ }^{4,5}$ It is therefore necessary to study systematic errors as well as the daily variations in patient positioning, so that the accuracy of beam placement and margins delineating the treatment area can be determined. Placement errors are determined by comparing portal images with simulation images. In most cases the comparison is performed visually by radiologists, although much work has also been done in developing completely automated or semi interactive comparison methodologies based on image segmentation and pattern recognition techniques. ${ }^{6-8}$

\subsection{Limitations of Megavoltage X-ray Imaging}

Imaging in the megavoltage x-ray range has some inherent limitations which fundamentally limit the image quality in terms of contrast and spatial resolution. There is also a tradeoff between patient dose and image quality, which becomes all the more critical because of the large doses involved. The different aspects of image quality that are affected during megavoltage x-ray imaging are described below. 


\subsubsection{Spatial Resolution}

Compton scattering accounts for most of the x-ray interactions in the $2 \mathrm{MV}$ to $25 \mathrm{MV}$ range. ${ }^{9}$ The Compton scattered photons in the patient, themselves undergo multiple Compton interactions. As a result of these multiple Compton scattering events, there is a significant lowering of the spatial resolution.

\subsubsection{Contrast Resolution}

Megavoltage x-ray images inherently suffer from poor contrast resolution. Unlike diagnostic xray energies, where most of the interactions are photoelectric, high energy x-ray interactions are dominated by Compton scattering. The photoelectric coefficient is highly dependent on the atomic number $(\mathrm{Z})$ of the interacting material i.e. it varies approximately as the third power of $\mathrm{Z}$, while the photoelectric cross section varies as the fifth power of $\mathrm{Z} .{ }^{10}$ Due to this strong $\mathrm{Z}$ dependence, the difference in x-ray attenuation by bone and tissue is quite high. As a result of this high difference, diagnostic x-ray images show high contrast. On the other hand, the Compton effect is relatively independent of $\mathrm{Z} .{ }^{10}$ The contrast seen in megavoltage images is due to small differences in tissue density, which subsequently result in poor contrast resolution. ${ }^{11}$

Furthermore, the photons generated due to multiple Compton scattering are widely deflected with respect to the direction of the primary beam. Therefore, rather than contributing to useful image information, these Compton scattered photons are seen as random noise in the image. This random noise or "fog" further lowers the image contrast. 


\subsubsection{Requirements of a Portal Imaging System}

Given the fundamental limitations of megavoltage x-ray imaging, it is obviously unrealistic for a portal imaging system to achieve spatial and contrast resolution comparable to diagnostic $\mathrm{x}$-ray images. The utility of a portal image lies in its ability to help identify errors in beam placement and patient alignment. These errors are determined by comparing the relative positions of large bony structures in simulation and portal images. These bony structures are invariant in position with respect to each other over the entire course of radiation treatment. Very fine structures consisting of soft tissue cannot serve as suitable landmarks because there is a possibility that they may lose shape or shift with respect to each other as the treatment progresses. Thus, the spatial resolution of a portal imaging system need only be high enough to clearly delineate the bony landmarks (typically, slightly over $1 \mathrm{cy} / \mathrm{mm}$ ). Higher resolutions (e.g. $2.5 \mathrm{cy} / \mathrm{mm}$ and above) are neither easily achievable nor required.

The other factor that determines image quality is the contrast resolution. High contrast resolution improves the physician's ability to delineate anatomical landmarks with respect to beam placement. ${ }^{11,12}$ As discussed in the previous section, contrast resolution in megavoltage images is severely limited due to the dominance of Compton interactions at these high x-ray energies. Operating within this constraint, it is nonetheless essential that the portal imaging system have the highest possible contrast resolution.

\subsection{Portal Imaging Modalities}

Traditionally, megavoltage x-ray imaging has been performed using portal film. In recent years however, electronic portal imaging devices (EPIDs) are being used in an increasing number of 
clinics. Although high contrast portal film is the current gold standard for image quality, EPIDs provide all the advantages of digital imaging. The following sub sections describe some of the current commercial and experimental portal imaging technologies.

\subsubsection{Portal Film}

Portal film is the most commonly used modality for portal imaging. It is relatively inexpensive and gives the highest resolution among all megavoltage imaging technologies $(2 \mathrm{cy} / \mathrm{mm})$. Portal film works on the same principle as ordinary radiographic film i.e. the image is produced due to the interaction of x-ray photons with a silver halide emulsion. Two types of portal film, conventional and high contrast, are used clinically.

\subsubsection{Conventional Portal Film}

Conventional portal film consists of a metal plate/film combination. Usually, the film is sandwiched between two plates of lead, copper, brass or aluminum. The front-end metal plate acts as a build up layer that increases the quantum detection efficiency (QDE) of the film. The film is thus exposed to the photon/electron shower generated in the build up plate. The plate also shields the film from brehmstrahhlung and scattered electrons generated as the beam exits the patient. $^{13}$

A major drawback of conventional film is low image contrast. This low contrast results primarily from the low QDE of film and its relatively larger film grain size. Theoretically, the QDE can be improved by increasing the thickness of the buildup plate. However, such an increase in plate thickness results in a significant loss of spatial resolution. The larger grain size is used to 
compensate for the inherent low sensitivity of the film to the photon/electron shower. Large grain size increases the granularity of the film, which increases the noise. This increased granularity noise causes a significant decrease in the SNR,${ }^{14}$ which makes it difficult to resolve low contrast structures.

\subsubsection{High Contrast Portal Film}

As discussed in section 3.3, high contrast resolution is critical in ensuring accurate beam positioning. It has been shown that significantly higher contrast can be achieved in portal film when the film is also exposed to light emitted from fluorescent intensifying screens placed in contact with the metal screen, ${ }^{15,16}$ This principle is used in the manufacture of high contrast portal film. Additionally, commercial high contrast film like Kodak EC-L film uses very fine grain cubic crystals which are an order of magnitude smaller in size as compared to the grain size of conventional portal film. EC-L film also has a much narrower distribution of grain sizes compared to conventional film. This narrow distribution results in higher contrast, while the lower film granularity contributes to increased SNR, which further improves the contrast resolution. ${ }^{15}$

High contrast portal film is currently the gold standard for portal image quality. However, it is more expensive as compared to conventional portal film. Also, owing to its sharp dose response characteristic, which results in higher contrast, high contrast film has a much narrower latitude compared to conventional portal film. Due to this narrow latitude, repeated filming may be required in order to obtain a clinically acceptable image, which would obviously increase the 
patient dose. This drawback therefore restricts the use of high contrast film in routine clinical practice.

\subsubsection{Electronic Portal Imaging Devices (EPIDs)}

A number of electronic portal imaging technologies have been developed. Some of these technologies are already commercially available while others are still under development.

EPIDs have the advantage of real time or near real time digital imaging, which is not possible with film. Some of the current commercial and experimental EPID technologies are described below.

\subsubsection{Fluorescent Screen - TV Camera based Systems}

These are the most common EPIDs in commercial use. They consist of a metal plate fluorescent phosphor screen combination, a $45^{\circ}$ mirror and a TV camera. ${ }^{17}$ Megavoltage x-ray photons interact with the build up plate to produce a high energy electron shower, which in turn produces fluorescence in the phosphor screen. The light produced at the screen is deflected by the $45^{\circ}$ mirror and is collected by a lens coupled to the TV camera.

The mirror is used to ensure that the camera and the associated readout electronics do not lie in the direct path of the high energy x-rays.

TV camera based systems have the advantages of near real time imaging and relatively lower radiation exposure to the image acquisition electronics. The main limitation of TV camera based EPIDs is their low light collection efficiency, which creates a secondary quantum sink (i.e. in addition to the $\mathrm{x}$-ray quantum sink), resulting in a significant loss of DQE. Another drawback is 
that the system tends to be bulky, owing to the large optical path. The bulky design makes patient set up difficult.

\subsubsection{Liquid Ionization Detector ${ }^{17}$}

The liquid ionization based EPID consists of a matrix of liquid filled ionization chambers coupled to a metal build up plate. ${ }^{18-21}$ Megavoltage photons interact with the build up plate, producing high energy electrons which ionize the liquid in each element of the detector. The signal from the detectors is read out by the electronics and a corresponding two dimensional image is reconstructed.

Liquid ionization based EPIDs give near real time images with image quality comparable to portal film. ${ }^{19}$ They also have a very good dose response and hence are used extensively in portal dosimetry. ${ }^{22-25}$ The major limitation of these EPIDs is that they have to compromise between spatial resolution and image acquisition speed. ${ }^{21}$ Slower acquisition improves the resolution but increases the patient dose.

\subsubsection{Scintillation Crystal - Photodiode Detector}

These EPIDs consist of a linear array of zinc tungstate scintillation detectors which are optically coupled to a photodiode and data acquisition electronics. ${ }^{26}$ The image is acquired by scanning the array across the radiation field. Megavoltage x-ray photons interact with the scintillation crystals and create high energy electrons that pass through the scintillator volume to produce visible light. The visible light is detected by the photodiodes and read out by the electronics. 
The scanning geometry has the advantage of scatter reduction. Another advantage of this technology is that since the zinc tungstate crystals are optically transparent, the detector can be relatively long $(\sim 25 \mathrm{~mm})$ in the direction of the $\mathrm{x}$-ray beam, thus enabling the system to achieve a very high QDE. This high QDE enables the system to take images at a lower dose. However, this advantage is offset by the fact that the EPID is a scanning system and hence the dose required for a full field scan is relatively high i.e. of the order of $20 \mathrm{cGy}$ for a $17 \times 17 \mathrm{~cm}$ field. ${ }^{17}$

\subsubsection{Amorphous Silicon}

TV camera based EPIDs suffer from low light collection efficiency, which reduces their image quality. They are also bulky and hence awkward to use in a clinical environment. An experimental technology that uses an amorphous silicon flat panel detector array coupled to a metal plate - phosphor screen combination, has shown great promise in overcoming these difficulties. The device consists of a metal build up plate, a phosphor screen, a two dimensional array of amorphous Si photodiodes, attached to the phosphor screen and the associated read out electronics. ${ }^{27,28}$ The array acts as a large area light sensor and records the light signal produced in the phosphor screen due to megavoltage x-ray interactions in the metal - phosphor screen combination.

Unlike TV camera - phosphor screen based EPIDs, the amorphous Si based technology has the advantage of being x-ray quantum limited rather than light quantum limited. The elimination of the secondary quantum sink significantly increases the $\mathrm{DQE}(\mathrm{f})$, thus improving image quality. The spatial and contrast resolution is comparable to current EPIDs. ${ }^{29}$ The flat panel EPID is also less bulky and hence easier to use in a clinical environment. The limitations of this technology 
are array size and non-uniform pixel performance. Present sensors are of the order of $26 \times 26$

$\mathrm{cm} \cdot{ }^{27}$ Current fabrication technology has been unable to produce large size arrays that can be used to cover the full field of the irradiated area, though much work is being done in this direction. Another problem faced by flat panel EPIDs is that the sensors and acquisition electronics lie very close to the detector and hence receive large amounts of high energy radiation. The large radiation doses can significantly degrade the performance of the EPID. This problem is absent in TV camera based EPIDs as explained in section 4.2.1.

\subsubsection{Kinestatic Charge Detector (KCD)}

The KCD is a scanning portal imaging system, being developed at the University of Tennessee. ${ }^{30}$ It uses a high pressure xenon gas filled chamber with a slot beam that scans across the irradiated area. Preliminary experiments with 100 atmospheres of xenon have demonstrated that the KCD has a high QDE (30\%) and a spatial resolution of $1 \mathrm{cy} / \mathrm{mm}$. The high QDE results in high contrast resolution at significantly lower doses compared to commercial EPIDs.

Due to its low dose requirements, the $\mathrm{KCD}$ is likely to be a promising system for pre-treatment verification of beam placement. Its major limitation is the fact that it is a scanning system. Therefore it probably has limited use for imaging during the radiotherapy procedure.

\subsubsection{Film Vs EPIDs: The Case for Digital Portal Imaging}

Despite the development of new digital imaging technologies, portal film continues to be the most commonly used portal imaging modality. Portal film is relatively inexpensive and gives the highest possible spatial resolution. High contrast film, which is considered the gold standard in 
portal imaging, gives excellent spatial as well as contrast resolution. One of the major limitations of film is its narrow latitude, which makes it difficult to determine the filming dose required for patients of different thicknesses. Due to this limitation it may be necessary to take more than one exposure in order to obtain a clinically acceptable image, thus exposing the patient to more radiation. Other drawbacks include difficulties in archival storage of film images and the fact that film images are much more prone to deteriorate with time.

EPIDs on the other hand, not only have a much larger latitude but also possess all the advantages of digital imaging i.e. ability to process, transmit and store images without loss or deterioration with time. Their main drawback is poor image quality as compared to film. However, the use of EPIDs is on the increase because of their numerous advantages over film and with the rapid developments taking place in EPID technology it is very likely that in the near future, they will equal the performance of film in terms of spatial and contrast resolution.

\subsection{Motivation for Use of a Transparent Scintillator}

\subsubsection{QDE}

All of the area detector based EPID technologies described above with the exception of TV camera based EPIDs, are x-ray quantum limited because the x-ray detectors used in these systems have a very low QDE at high energies. TV camera based systems are light quantum limited owing to their low light collection efficiency. However, the detectors used in TV camera based systems also encounter the problem of low x-ray detection efficiency. To improve the low QDE, a metal build up plate is coupled to the x-ray detector. Megavoltage x-rays impinging on 
the build up plate generate high-energy electrons which can be more easily stopped by the x-ray detector than the X-rays themselves. Increase in the thickness of the phosphor screen or the build up plate increases the detection efficiency and SNR, but causes a significant decrease in spatial resolution. ${ }^{31,32}$ TV camera based EPIDs although light quantum limited, also face this problem of a compromise between QDE and spatial resolution. As opposed to this, a thick transparent CsI (Tl) scintillator provides a longer path for X-ray interactions and thus improves the QDE at the detector by an order of magnitude as compared to the existing EPID technologies (i.e. over $20 \%$ as compared to $2 \%$ ).

\subsubsection{Contrast Resolution}

The high QDE of the CsI (Tl) detector greatly improves the contrast resolution. Higher detection efficiency also means that more image information is acquired and a large amount of digital contrast enhancement can be used to improve the perceived image contrast.

\subsubsection{Spatial Resolution}

As explained in section 5.1, current EPID technologies have to compromise between detection efficiency and spatial resolution. The spatial resolution of phosphor screen based EPIDs decreases significantly with screen thickness. This problem is much less severe in case of a transparent scintillator with an optically flat exit window because the light produced in the scintillator travels in straight lines through the detector material and gets collected by the camera lens. Therefore, the spatial resolution of a transparent scintillator is much less dependent on the detector thickness, which means that any reasonable thickness can be used without the need to compromise between spatial resolution and contrast resolution. 


\section{CHAPTER 2}

\section{Basic Physics and Electronics}

\subsection{Interaction of High Energy X-rays with Matter}

An X-ray beam passing through an object undergoes 5 types of interactions

1. Photoelectric Effect

2. Compton Effect

3. Pair Production

4. Rayleigh scattering (Coherent scattering)

5. Photonuclear interactions

In x-ray imaging, the first three interactions are important since they result in energy transfer and energy absorption. Rayleigh scattering is elastic and occurs without any energy loss. However, Rayleigh scattering contributes to image contrast because the scattered flux tends to miss the $\mathrm{x}$ ray detector. Photonuclear reactions are significant for photons that have very high energies i.e. above several $\mathrm{MeV}$ and hence these interactions are not very important for $\mathrm{x}$-ray imaging applications.

When a photon enters an object, part of its energy is radiated from the medium as scattered radiation while part of it is converted into kinetic energy of high speed electrons and positrons. These high speed particles lose most of their energy by collisions with other particles in the medium and the remaining energy is lost by emitting bremsstrahlung. Depending upon the 
photon energy and the $\mathrm{Z}$ of the interacting medium, $\mathrm{x}$-ray interactions are dominated by the photoelectric effect, the Compton effect or pair production.

\subsubsection{Photoelectric Effect}

In the photoelectric process, a photon of energy hv interacts with an atom and is completely absorbed, causing the atom to eject a bound electron from the $\mathrm{K}, \mathrm{L}, \mathrm{M}$ or $\mathrm{N}$ shell. The ejected electron is called a photoelectron, which has an energy equal to $h v-E_{B S}$, where $E_{B S}$ is the binding energy of the shell. An electron from an outer shell jumps to take the place of the ejected electron, leaving the atom in an excited state. To return to the ground state, the atom emits characteristic radiation and Auger electrons.

Photoelectric absorption is greatly increased when the photon interacts to eject the inner shell electrons, which are more tightly bound. The photoelectric process is most likely to occur if the energy of the photon is just greater than the binding energy of the electron. The photoelectric cross section, i.e. the probability of a photoelectric interaction, varies with photon energy as $(1 / \mathrm{h} v)^{3}$ and the photoelectric coefficient per electron or per gram varies approximately as $(\mathrm{Z})^{3}$. It is therefore clear that photoelectric interactions are dominant at lower photon energies like those used for diagnostic x-ray imaging. ${ }^{33}$

\subsubsection{Compton Effect}

In a Compton interaction, an incident photon collides with an electron and transfers part of its energy to the electron. As a result of the collision, the photon is deflected from its original path and the electron recoils with a finite kinetic energy and momentum. Energy and momentum 
between the photon and the electron are conserved. Since x-rays in the megavoltage range used in radiotherapy $(1-10 \mathrm{MeV})$ interact in matter mostly by the Compton effect, the Compton effect is discussed in some detail.

The analysis of a Compton collision is usually performed by assuming the electron to be unbound and at rest. As shown in figure 2-1, a Compton interaction occurs when a photon of energy hv and momentum $h v / c$, collides with an electron, assumed to be at rest.

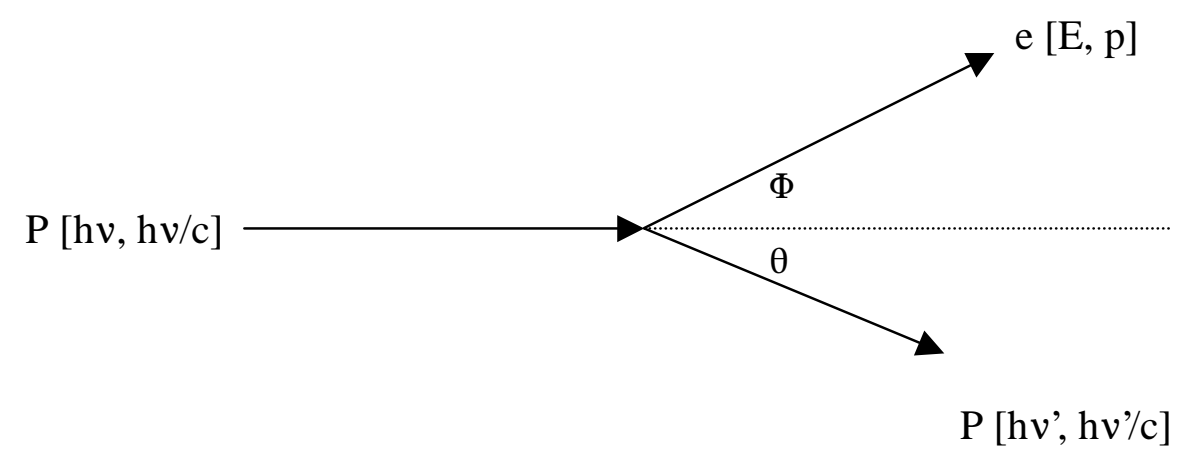

Figure 2-1. Compton collision between a photon of energy hv and an electron assumed to be stationary. After collision, the electron is deflected at an angle $\Phi$ and acquires an energy E. The photon is deflected at an angle $\theta$ and retains an energy equal to hv'.

As a result of the collision, the photon and the electron are deflected by angles $\theta$ and $\Phi$ respectively. The electron acquires an energy $\mathrm{E}$ and a momentum $\mathrm{p}$, while the photon retains an energy equal to $h v^{\prime}$ and a corresponding momentum of $h v^{\prime} / \mathrm{c}$. Using the laws of conservation of energy and momentum, the following relationships can be derived. ${ }^{9}$

$$
E=h v \cdot \frac{\alpha(1-\cos \theta)}{1+\alpha(1-\cos \theta)}
$$




$$
h v^{\prime}=h v \cdot \frac{1}{1+\alpha(1-\cos \theta)}
$$

where $\alpha$ is the ratio of the energy of the photon $(\mathrm{h} v)$ to the rest energy of the electron $\left(\mathrm{m}_{0} \mathrm{c}^{2}\right)$. If the energy is expressed in $\mathrm{MeV}$, the rest energy of the electron is $0.511 \mathrm{MeV}$.

A rough idea of the energy deposition due to Compton interactions as a function of the incident photon energy, can be obtained by considering a special case of equation 2.1.2-1. If the photon makes a head on collision with the electron, the electron travels straight forward $\left(\Phi=0^{\circ}\right)$, while the photon travels in the opposite direction $\left(\theta=180^{\circ}\right)$. In this case, the electron acquires maximum energy $\left(\mathrm{E}_{\max }\right)$ while the scattered photon retains minimum energy $\left(\mathrm{h} v_{\min }\right)$. The expressions for $E_{\max }$ and $h v_{\min }$ can be obtained by substituting the appropriate values of $\theta$ and $\Phi$ in equations 2.1.2-1 and 2.1.2-2.

$$
\begin{aligned}
& E_{\max }=h v \cdot \frac{2 \alpha}{1+2 \alpha} \\
& h v_{\min }^{,}=h v \cdot \frac{1}{1+2 \alpha}
\end{aligned}
$$

Equation 2.1.2-3 shows that in case of a low energy photon (e.g. $100 \mathrm{KeV}$ ), the maximum energy transferred will be a very small percentage of the photon energy because $\alpha<1$. On the other hand, if a high energy photon (e.g. $6 \mathrm{MeV}$ ) collides head on with an electron a significant percentage of the photon energy will be transferred to the electron. 
The probability of a Compton interaction with a free electron i.e. the Compton cross section per electron is calculated using the Klein-Nishina formula. The Klein-Nishina analysis, which assumes stationary and unbound electrons, shows that the Compton cross section per electron, denoted by ${ }_{\mathrm{e}} \sigma$, is independent of the atomic number $\mathrm{Z}$. ${ }_{\mathrm{e}} \sigma$ is relatively constant at energies below $0.01 \mathrm{MeV}$ and decreases proportional to $(1 / \mathrm{h} v)$ for higher photon energies. ${ }^{9}$

\subsubsection{Pair Production}

Pair production occurs when a high energy photon passes near the nucleus of an atom and is subjected to the strong Coulomb field of the nucleus. Pair production is an absorption process in which the photon is completely absorbed and gives rise to an electron and a positron. For pair production to occur, a minimum photon energy equal to twice the rest mass of an electron (1.022 $\mathrm{MeV}$ ) is required. If the incident photon has energy greater than $1.022 \mathrm{MeV}$, then this excess energy appears as kinetic energy of the electron and positron. The positron travels through the medium, exciting and ionizing atoms, until it recombines with a free electron and undergoes annihilation, producing two photons, each of $0.511 \mathrm{MeV}$, ejected in opposite directions to conserve momentum. The electron and positron do not necessarily receive equal kinetic energies. In fact, all distributions of the available energy are equally probable except for extreme ones where one particle gets almost all the energy. ${ }^{9}$

Pair production can only occur in a Coulomb force field and hence it usually occurs near the atomic nucleus. However, it can also occur with lower probability, in the field of an electron. This interaction is known as triplet production because the electron that provides the field also 
acquires significant kinetic energy during the interaction. Thus in triplet production, two electrons and a positron are emitted from the site of interaction.

The pair production coefficient per atom, denoted by ${ }_{a} \kappa$, is proportional to $\mathrm{Z}^{2}$, while the coefficient per electron or per gram varies as $\mathrm{Z}$. The atomic cross section for triplet production is small as compared to the nuclear pair production cross section coefficient. ${ }^{33}$ The ratio is given as

$$
\frac{{ }_{a} K_{\text {electrons }}}{{ }_{a} \kappa_{\text {nucleus }}}=\frac{1}{C Z}
$$

where $\mathrm{C}$ is an energy dependent parameter. $\mathrm{C}$ is equal to unity as $\mathrm{h} v \rightarrow \infty$, and it rises slowly with decreasing energy to $\cong 2$ at $5 \mathrm{MeV}$. Usually, the cross sections for pair production and triplet production are combined into a single cross section that is still referred to as the pair production cross section.

$$
\left(\frac{{ }_{a} \kappa}{\rho}\right)_{\text {pair }}=\left(\frac{{ }_{a} \kappa}{\rho}\right)_{\text {nuclear }}+\left(\frac{{ }_{a} \kappa}{\rho}\right)_{\text {electron }}
$$

The pair production cross section increases roughly logarithmically with increase in energy, above the threshold of $1.02 \mathrm{MeV}$ and gradually becomes independent of $\mathrm{h} v$ for very high energies (above $100 \mathrm{MeV}$ ). Thus, pair production is dominant in the $10 \mathrm{MeV}-100 \mathrm{MeV}$ range.

\subsection{Scintillation Detector Principles}

X-ray scintillators are one of the oldest techniques for detecting ionizing radiation. A scintillator converts the kinetic energy of the charged particles excited due to x-ray interactions into visible 
light that can be detected by a suitable light detector. Desirable properties for a scintillator include good sensitivity, high linearity and a high degree of transparency to the wavelength of its own emission so as to ensure good light collection. Commonly used scintillator materials are inorganic alkyl halides, and organic based liquids and plastics. Inorganic scintillators usually have higher light output and linearity, while organic scintillators tend to have faster response time and a linear dose response but significantly lower light output. Inorganic scintillator materials have high Z-values and high density, which favors their use as x-ray and gamma ray detectors. Organic scintillators are used mainly in dosimetry, beta spectroscopy and fast neutron detection. ${ }^{34}$

\subsubsection{Scintillation in Inorganic Crystalline Materials}

Inorganic scintillators are crystalline semiconductors or insulators. The outer electrons in these materials can occupy only two discrete energy bands. The electrons in the lower energy band or the valence band, are bound to the nucleus, while the electrons in the upper conduction band have sufficient energy to be able to freely migrate throughout the crystal lattice. The intermediate energy band is called the forbidden gap, which represents energy states that are not allowed for an electron in a pure crystal.

Absorption of energy in the crystal may cause an electron to jump from the valence band into the conduction band, leaving a positively charged hole in the valence band. The de-excitation of the electron occurs with the emission of a photon of energy equal to the width of the forbidden gap. In a pure crystal, the energy corresponding to the gap width is too high for the resulting photon to lie in the visible range. 
To enable the emission of a visible photon, small amounts of impurities, called activators, are added to the crystal. The addition of an activator modifies the energy band structure of the pure crystal, creating energy states in the forbidden gap as shown in figure 2-2. Since the energy difference between the activator created energy states and the ground state is less than the forbidden gap width, the transition of an electron from the activator excited states to the ground state can now give rise to a photon that lies in the visible range.

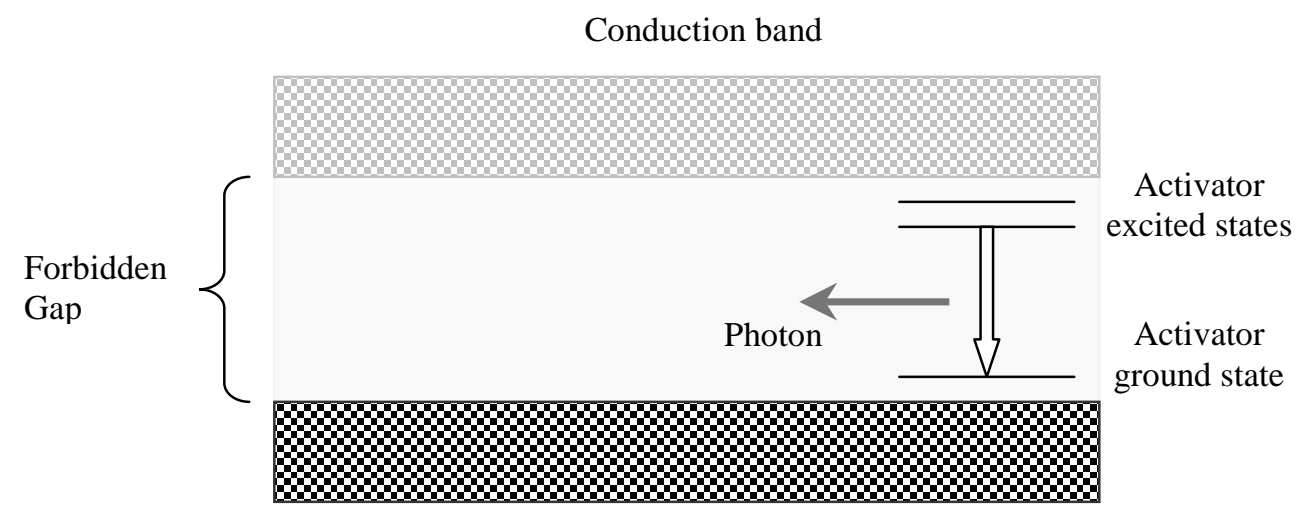

Valence band

Figure 2-2. Energy band structure and scintillation mechanism of an activated crystalline scintillator

The scintillation process takes place in the following manner. Energy is imparted to charged particles during x-ray interaction. A charged particle passing through the scintillator material transfers energy to electrons in the valence band, causing them to jump to the conduction band. This elevation of the electron causes the formation of a large number of electron-hole pairs. The positive hole drifts towards an activator site and ionizes it since the ionization energy of the impurity is less than that of a typical lattice site in the crystal. Meanwhile, the electron freely migrates through the crystal until it encounters such an ionized activator site, upon which the electron drops into this "electron trap". The electron traps lie at a slightly lower energy level than 
the conduction band as shown in figure 2-2. If a transition to the ground state is allowed from the energy level of the trap, de-excitation of the electron occurs. If the impurity is properly chosen there is a high probability that the emitted photon will lie in the visible light range.

A process called phosphorescence competes with the one described above. Sometimes an excited state gets created such that transition from this state to the ground state is forbidden. The electrons that fall in these states, require an additional increment of energy to raise them to a higher energy excited state from which de-excitation to the ground state is possible. This additional energy can be obtained from thermal excitation. The resulting light appears as background light or afterglow in the scintillator.

\subsubsection{Scintillation Properties of CsI (TI)}

Cesium iodide is an alkyl halide scintillator activated using thallium or sodium. It has high x-ray stopping power due to its relatively high density $\left(4.51 \mathrm{~g} / \mathrm{cm}^{3}\right)$ and high $\mathrm{Z}$ value.

It is relatively soft and malleable as compared to other inorganic scintillators like $\mathrm{NaI}(\mathrm{Tl})$, and can be cut and bent into various shapes without fracturing. It is soluble in water but less hygroscopic than $\mathrm{NaI}(\mathrm{Tl})$.

CsI (Tl) has the highest light output of all presently known scintillators. ${ }^{34}$ Its peak output is centered around $550 \mathrm{~nm}$ and is therefore, not well matched to photomultiplier tubes. However, with solid state detectors like CCD cameras and photodiodes, the high light output of CsI (Tl) can be effectively used to increase light collection efficiency. CsI (Tl) is a relatively slow scintillator and has an average decay time of about one microsecond. ${ }^{35}$ The transmission 
spectrum of CsI ( $\mathrm{Tl})$ is relatively resistant to radiation damage. It has been shown that high quality CsI (Tl) loses only about $14 \%$ of its light output for radiation doses up to $2.0 \times 10^{6}$ rad. $^{35}$

\subsection{CCD Based Imaging Systems}

In recent years, charge coupled device (CCD) based imaging systems have gained immense popularity in various areas like astronomy, digital radiography and TV camera systems. CCD based systems have several advantages over traditional electron beam scanned camera tubes. These advantages include ruggedness, compactness, lower power consumption and the ability to perform complex signal processing functions.

\subsubsection{CCD Operating Principles}

A CCD is basically a multi-gate MOSFET (metal oxide semiconductor field effect transistor) in which charge can be stored and transferred. The fundamental unit of a CCD is the MOS capacitor, which consists of a p-type or n-type $\mathrm{Si}$ layer, a $\mathrm{SiO}_{2}$ layer and a metal plate electrode acting as the gate. A voltage is applied to the gate to bias the CCD. For a p-type semiconductor, the gate voltage is positive while for an n-type semiconductor the gate voltage is negative. The two processes fundamental to the operation of a CCD are charge storage and charge coupling.

\subsubsection{Charge Storage}

Consider a p-type MOS device. Prior to the application of a bias, there is a uniform distribution of holes (majority charge carriers) in the semiconductor. When a positive voltage is applied to the gate, the holes are repelled from the region immediately beneath the gate, forming a depletion layer. The depth of this depletion layer is proportional to the gate bias voltage. If the 
gate voltage is increased till it reaches a certain threshold, the minority carriers (electrons) in the semiconductor get attracted towards the positively charged gate and form a thin inversion layer at the surface. When this inversion layer is negative, the CCD is called an n-channel CCD.

As the gate voltage is increased above the threshold voltage, more and more minority charge carriers continue to accumulate below the gate, increasing the charge density of the inversion layer. The increased charge density leads to an increase in the potential drop across the oxide

layer. Since this potential drop varies almost linearly with the gate voltage ${ }^{36}$ it is modeled as a potential well whose depth is proportional to the gate bias. It should be noted that the physical extent of the depletion layer is different from the depth of the potential well. The potential well is a purely hypothetical concept in which charge is represented as a liquid, the volume of which is proportional to the amount of charge present on the inversion layer. The depletion layer shrinks as more charge (in the form of minority carriers) is introduced. On the other hand, the "depth" of the potential well remains the same for a given gate voltage. With the introduction of more charge, the "well" merely fills up to a greater extent.

\subsubsection{Charge Coupling}

The concept of charge coupling in a CCD can be explained by considering the arrangement shown in figure $2-3,{ }^{37}$ which shows four closely spaced electrodes. Assume the threshold voltage to be slightly under $2 \mathrm{~V}$. The electrodes are always maintained at a potential above the threshold voltage to ensure that a potential well is formed beneath each electrode. Assume that some charge is stored initially in the potential well under the second electrode, which is biased to $10 \mathrm{~V}$. Now if the potential on the adjacent (third) electrode is increased to $10 \mathrm{~V}$, while the potential on 
the second electrode is decreased at a proportionate rate to $2 \mathrm{~V}$, the charge present in the second potential well will migrate to the third potential well.
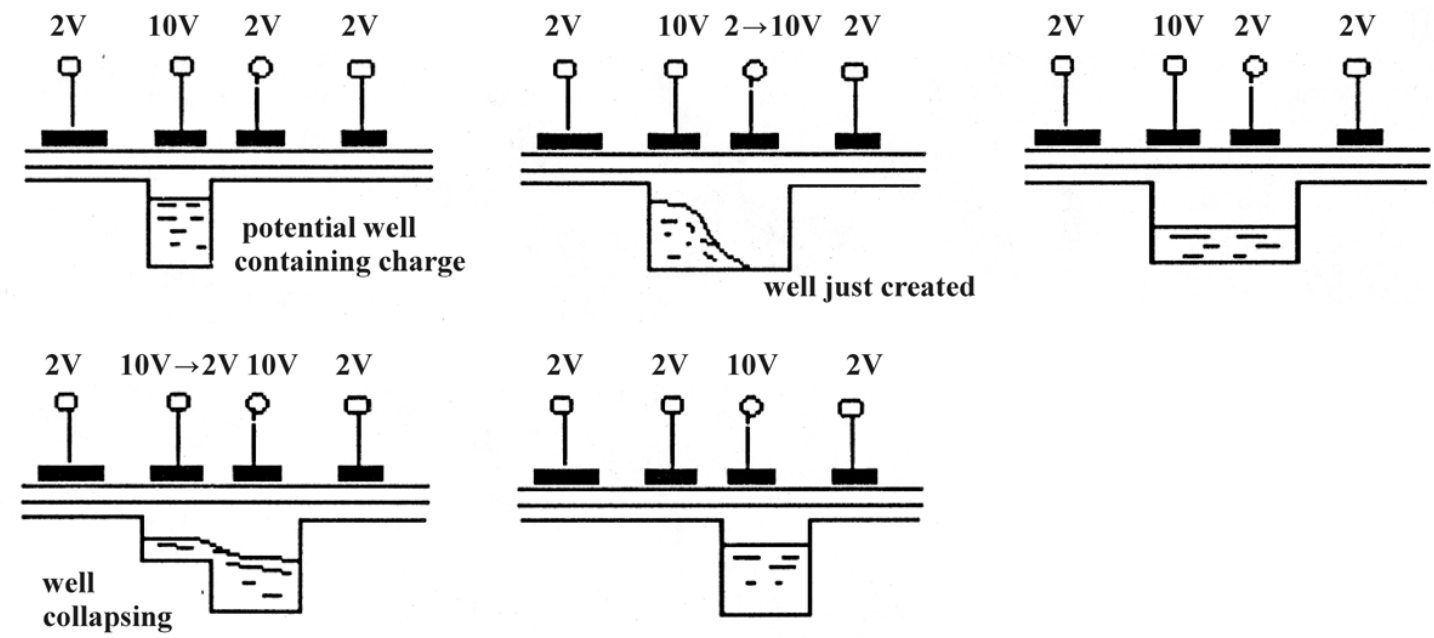

Figure 2-3. Movement of charge packets by clocking the CCD electrodes

In this manner, by applying successive voltages to the CCD electrodes, a charge packet can be propagated beneath the surface of the semiconductor. It is obviously not practical to access each electrode individually in a multi-gated CCD. This access problem is solved by grouping the electrodes and connecting each group to a different phase of a driving clock. The number of phases needed depends upon the detailed structure of the CCD. Usually two phase and three phase systems are used.

\subsubsection{Optical Imaging using CCDs}

Semiconductor materials have the property of absorbing electromagnetic radiation. Incident electromagnetic radiation may be absorbed and thereby stimulate the transition of electrons from 
one energy state to a higher energy state. This opto-electronic interaction is the basis of CCD image sensors.

\subsubsection{Absorption Processes in Semiconductors}

The outer shell electrons in semiconductors are present in one of three states, i.e. valence band electrons, conduction band electrons or electrons bound to localized impurity or defect centers. Absorption of radiation may result in the elevation of a valence band electron to the conduction band, giving rise to the formation of an electron - hole pair. This process is known as intrinsic absorption and forms the basis of radiation sensors in the visible range as well as some sensors in the infrared range. The cutoff wavelength for intrinsic absorption depends upon the energy gap and can be calculated using the relationship $\lambda_{\text {cin }}=h c / E_{B G}$, where $E_{B G}$ is the energy of the band gap.

Another absorption process called extrinsic absorption occurs when charge carriers bound to localized impurity or defect centers absorb energy. In this case the absorption process generates only one type of charge carrier. The transition resulting from extrinsic absorption may involve an electron in a neutral donor site being emitted into the conduction band or the capture of an electron by an ionized acceptor in the valence band. The latter interaction may be considered as the emission of a hole from the valence band. The energy transition in extrinsic absorption is lower than that in case of intrinsic absorption since it depends on the relatively smaller energy difference between the impurity energy level and a band edge. The difference in energy between the impurity level and the band edge is called the ionization energy $\mathrm{E}_{\mathrm{i}}$. Similar to intrinsic absorption, the cutoff wavelength for extrinsic absorption can be calculated as $\lambda_{\text {cex }}=\mathrm{hc} / \mathrm{E}_{\mathrm{i}}$. 
Due to the smaller energy gap, the wavelengths detected by extrinsic absorption lie in the infrared range. Since extrinsic absorption occurs only at the impurity sites, it is characterized by a significantly lower quantum efficiency compared to intrinsic absorption.

\subsubsection{Dark Current}

In the absorption processes described above, the electrons or holes receive energy from incident photons and are thus able to make the transition to a higher energy state. However, this transition can also be made by minority carriers that absorb thermal energy when the semiconductor is at a non-zero temperature. This process of thermal absorption creates an undesirable source of charge known as dark current that corrupts the useful signal. The magnitude of the dark current is proportional to the device temperature. Obviously, to minimize the dark current the semiconductor device should be maintained at a very low temperature during operation.

\subsubsection{CCD as an Image Sensor}

When electromagnetic radiation is incident on a CCD, minority carriers are generated within the CCD structure. The clocking of the CCD electrodes is stopped for a short time, with only the first electrode turned on. The optically generated minority carriers accumulate in the potential well created by this electrode, with the charge proportional to the local light intensity.

In a CCD image sensor a two dimensional array of CCD elements is used to record an image that is focussed on the array using an optical system. Any one set of electrodes (e.g. $\Phi 1$ ) is held at a positive clock voltage, creating a potential well under these electrodes, while the electrodes clocked by the other phases are held at zero volts or a small constant potential. The minority 
carriers generated due to the absorption of incident light photons are collected under each $\Phi 1$ electrode. The charge that is collected over a period of time called the integration time, is proportional to the local light intensity. Thus, the pattern of charge that collects under the electrodes is a replica of the light intensity across the original image. At the end of the integration period, this charge pattern is read out at a sufficiently high clock frequency. As shown in figure 2-4, the two dimensional array consists of parallel registers. The data from each parallel register is transferred to the serial register for read out. 


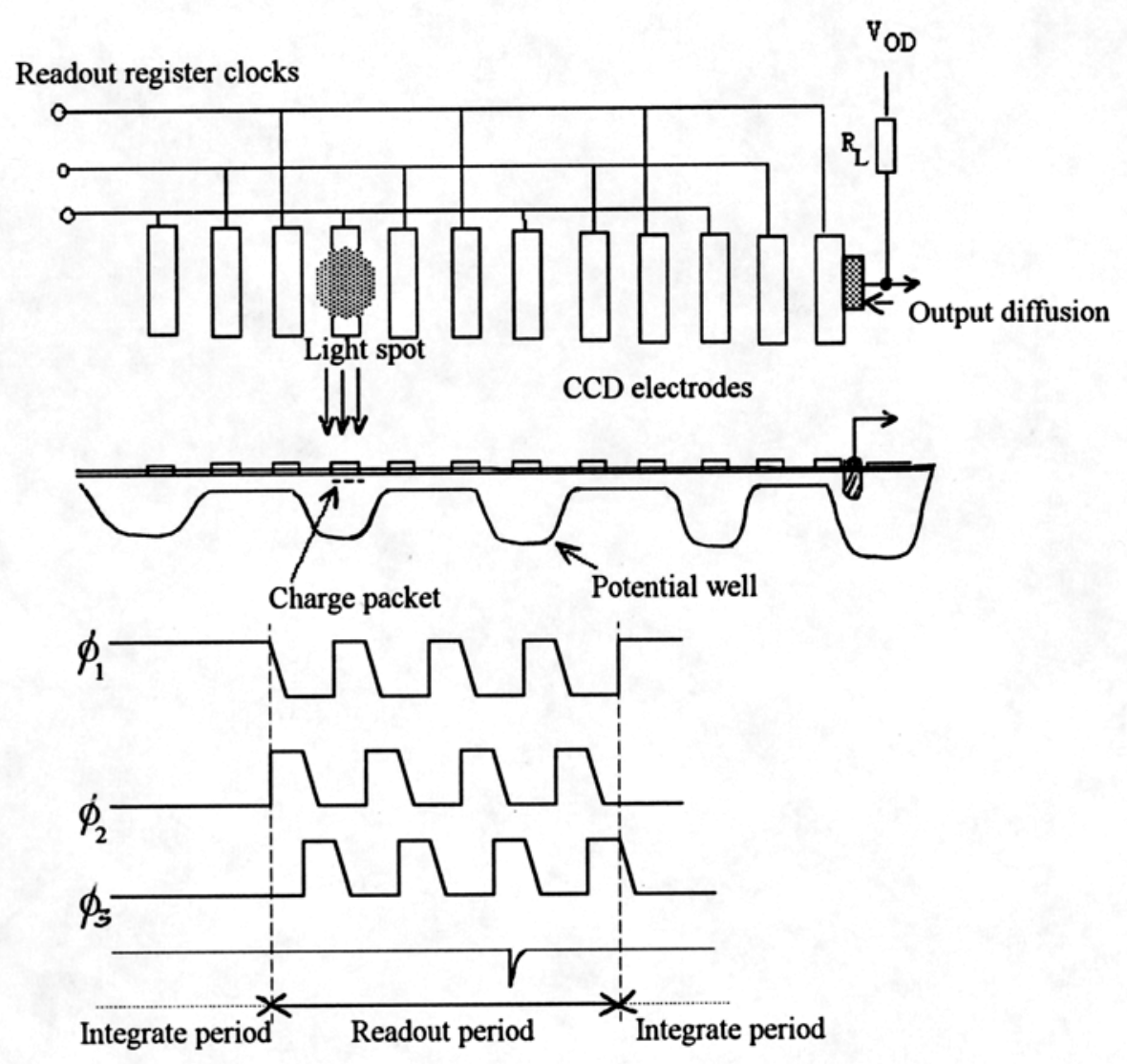

(a)

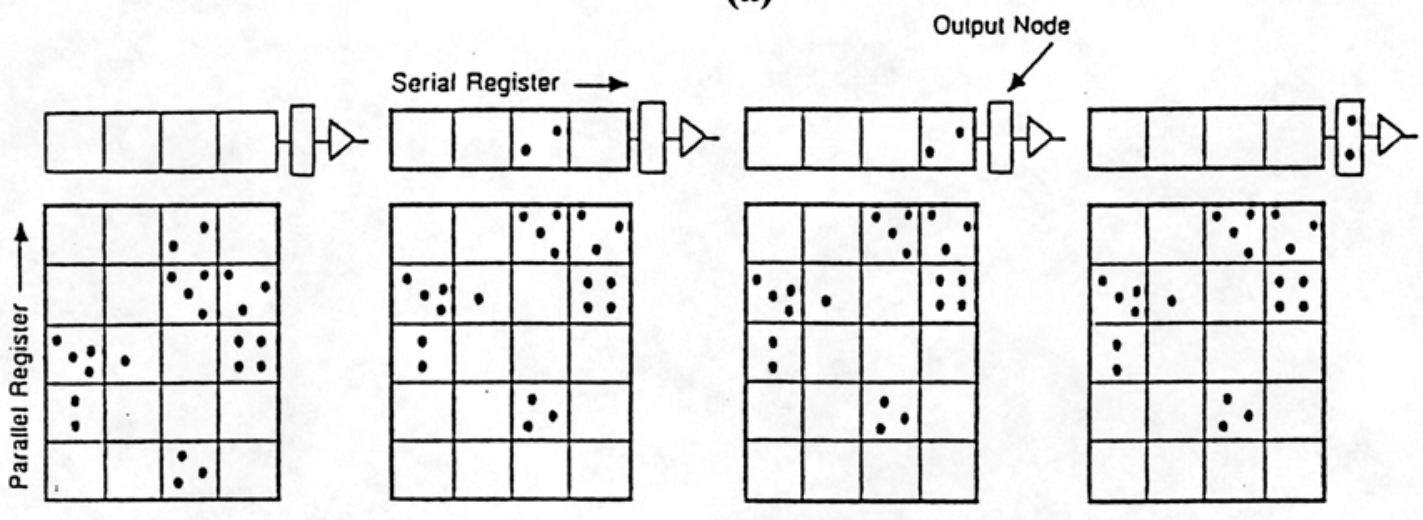

(b)

Figure 2-4. a) Basic structure and operation of a $\mathrm{CCD}$ imaging array. b) $\mathrm{CCD}$ readout 
The clock frequency for the electrodes needs to be sufficiently high in order to avoid image smear, which is caused by spurious charge picked up by the signal charge packets during the readout period if light is still incident on the array. In the Astromed ${ }^{\circledR} \mathrm{CCD}$ camera used for this research project, the problem of image smear is eliminated by using a mechanical shutter that can be closed before the readout begins.

\subsubsection{Basic Properties of a CCD Image Sensor}

\subsubsection{Spatial Resolution}

The spatial resolution of the CCD is limited mainly by the pixel size. The geometric modulation transfer function (MTF) which is the basic spatial response of an array of sensors, gives a measure of the spatial resolution of the CCD array. For a spatial response which is a rectangular function of width $e$ for each resolution element in the array, the geometric MTF is given by

$$
\mathrm{MTF}_{\text {geom }}=\frac{\mathrm{d}}{\pi \mathrm{se}} \sin \left(\frac{\pi \mathrm{se}}{\mathrm{d}}\right)
$$

where $s$ is the normalized frequency of input and $d$ is the sensor pitch. ${ }^{36}$

The other factors that determine the spatial resolution of CCD image sensors are the charge transfer efficiency and the carrier diffusion degradation. Since the charge packets must undergo thousands of transfers, low charge transfer efficiency may cause a fall in the MTF at higher frequencies. The other effect that may potentially lower the spatial resolution is the diffusion of charge carriers before they are collected. 


\subsubsection{Dark Current}

All semiconductor light measuring devices suffer from the presence of dark current, which appears along with the signal as additive noise. For CCD imagers, the dark current is measured in electrons per pixel per second. The origin of dark current was explained in section 3.2.2. Dark current is highly temperature dependent, decreasing approximately by a factor of 2 for every $10^{\circ} \mathrm{C}$ fall in temperature. Thus, the cooling of an imaging array significantly reduces the noise due to dark current. Cooling may be done thermoelectrically or by using liquid nitrogen as in case of the Astromed $^{\circledR}$ system.

\subsubsection{Sensitivity}

Sensitivity is the lowest signal level that can be detected by the imaging device. The sensitivity of a CCD imaging array depends upon the spectral response of the CCD and the system noise level. Since the spectral response of Si covers a very wide range from soft $\mathrm{x}$-rays $(0.1 \mathrm{~nm})$ to infrared $(1000 \mathrm{~nm})$, the CCD offers very high sensitivity. A measure of the spectral response can be obtained by calculating the average quantum efficiency $(\mathrm{QE})$ which is the ratio of the average number of light quanta detected to the number incident.

The other factor that determines the sensitivity is system noise. The noise sources in a CCD imager include photon noise, dark current, electronic noise (shot noise) and systematic spatial noise due inter-pixel response variations.

\subsubsection{Dynamic Range}


The dynamic range of a CCD is determined by the maximum charge handling capacity of the CCD at the upper end and by the noise sources at the lower end. The dynamic range is expressed in terms of possible signal levels in the device. The upper limit of the dynamic range determines the maximum charge that can be held in a potential well. If this limit is exceeded as may happen due to overexposure or while imaging a particularly bright spot in the image, an optical overload occurs which causes the excess carriers to spread into the adjacent pixels of the array. The amount of overload determines the extent if this spread. This overload phenomenon is called blooming. Blooming results in a localized "white out" in an image causing loss of information.

\section{CHAPTER 3}

\section{Theoretical Analysis}

\subsection{Introduction}

In recent years, a number of electronic portal imaging systems have been developed for the verification of beam placement and patient positioning during radiotherapy. ${ }^{17,38}$ Currently, TV camera based portal imaging systems are the most commonly used technology. Considerable work is also being done in the development of amorphous silicon based flat panel EPIDs. ${ }^{27-29}$ As 
explained in chapter 1, both the above technologies use detectors that have low quantum detection efficiency (QDE). In addition, TV based EPIDs also suffer from poor light collection efficiency, which further degrades image quality. Attempts have been made to improve the QDE by increasing the thickness of the phosphor screen or the metal buildup plate. However, it has been shown that although increased thickness of the screen or metal plate improves the QDE, there is a significant decrease in the spatial resolution. ${ }^{39}$ In order to avoid this compromise between QDE and spatial resolution we have constructed an EPID that uses a $12.7 \mathrm{~mm}$ thick transparent CsI (Tl) scintillator coupled to a liquid nitrogen cooled CCD camera. ${ }^{40,41}$ The use of a thick transparent scintillator provides a longer path for x-ray interactions in the detector and thus improves the QDE at the detector by an order of magnitude compared to the existing EPID technologies (i.e. over $20 \%$ as compared to $2 \%$ ). Since the detector is transparent, light rays produced at a point in the scintillator can exit along straight lines and be focussed back to a point on the CCD using the lens, which ensures that the spatial resolution is not significantly reduced even if the crystal thickness is increased to improve the QDE.

The experimental evaluation of the spatial resolution, contrast resolution and light glare of the CsI (Tl) - CCD system is described in chapter V. ${ }^{42}$ In this chapter, the theoretical performance of a CsI (Tl) - CCD portal imaging system is determined, using Monte Carlo simulation to calculate the QDE, the zero frequency detective quantum efficiency (DQE) and the signal to noise ratio (SNR). Analysis is performed for different thickness combinations of CsI (Tl) and leaded glass radiator to determine the optimal thickness combination. This work also aimed at calculating the frequency dependent DQE, denoted as DQE(f), for a proposed portal imaging system which uses 
a $12.7 \mathrm{~mm}$ CsI $(\mathrm{Tl})$ crystal coupled a PixelVision ${ }^{\circledR}$ back surface illuminated, thinned CCD camera having a light collection efficiency of $90 \%$.

It should be noted that the analysis performed here considers only the x-ray and light quantum noise and does not account for noise due to radiation directly hitting the CCD or the noise generated in the CCD and its associated readout electronics. As such, this analysis is therefore an indication of the upper limit of performance that can be reached using the present and proposed designs. Having stated this limitation, it should be noted that the effect of noise contributed by the CCD is relatively small, especially for a modern cooled CCD. e.g. typically the CCD dark current noise for a thermoelectrically cooled CCD is of the order of 1-2 electrons per pixel per second. ${ }^{43}$ The readout noise for slow scan cameras is about 15 electrons (rms) and about 40

electrons (rms) for frame transfer devices. ${ }^{43}$ In megavoltage imaging, the CCD read out noise is significantly lower than the noise due to stray radiation hitting the camera. However, the effect of this radiation noise can be reduced significantly by using adequate lead shielding around the $\mathrm{CCD}^{44}$ On the other hand, the effect of $\mathrm{x}$-ray and light quantum noise dominates the SNR transfer characteristics of a CsI (Tl) - CCD system.

\subsection{Background}

\subsubsection{DQE as a Measure of System Performance}

Detective quantum efficiency (DQE) is a commonly used measure for evaluating the performance of radiographic imaging systems. The DQE of a system is a measure of the SNR transfer efficiency of a system. For linear, shift invariant systems, the DQE can be calculated as the ratio of the square of the output SNR to the square of the input SNR. ${ }^{45}$ DQE indicates how 
efficiently the system transfers the input SNR. The input SNR is the ratio of the number of incident $\mathrm{x}$-ray photons to the random fluctuation in the counting statistics of these photons. In a practical system, the output SNR is always less than the incident SNR, due to the less than ideal (i.e. 100\%) QDE and the addition of noise. Therefore, the DQE of a practical radiography system will always be less than $100 \%$ and will depend upon the systematic and random noise introduced at various stages in the imaging system. The DQE is also dependent on the spatial frequency response of the imaging system. The frequency dependent DQE, which will be referred to as DQE (f), can be approximated by multiplying the zero frequency DQE defined above, with the square of the modulation transfer function (MTF) of the system. ${ }^{45}$

\subsubsection{System Description}

A schematic diagram of the CsI (Tl) - CCD portal imaging system is shown in figure 3-1. The system consists of a $12.7 \mathrm{~mm}$ thick, $20 \mathrm{~cm}$ diameter CsI (Tl) scintillator crystal that acts as the $\mathrm{x}$ ray detector. The scintillator is coupled to an Astromed ${ }^{\circledR}$ cooled CCD camera using a high speed lens.

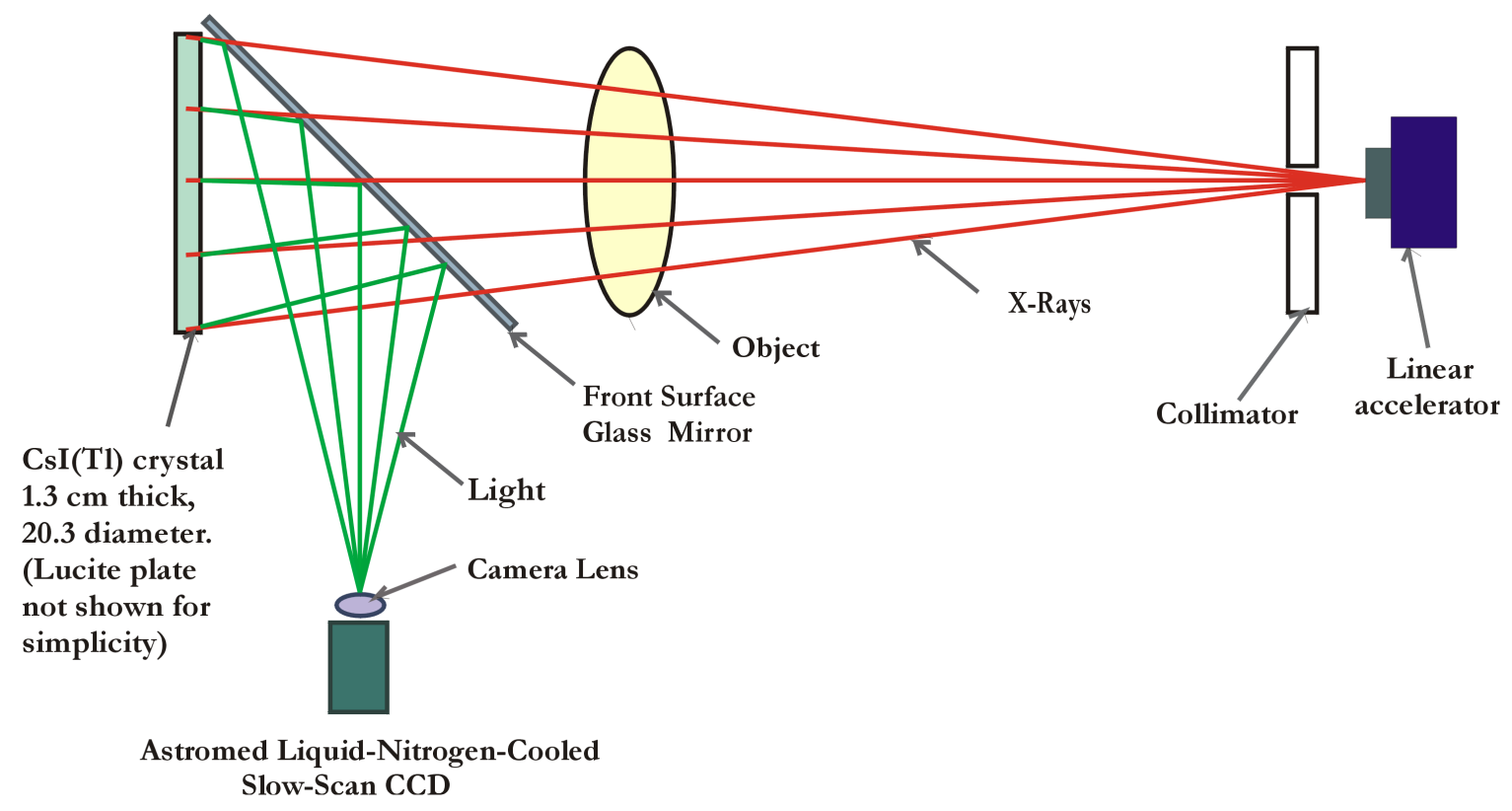


Figure 3-1. Experimental arrangement for the CCD-CsI (Tl) based portal imaging system.

In the actual prototype EPID, a $25 \mathrm{~mm}$ Lucite ${ }^{\circledR}$ plate (not shown in the figure) is coupled to the front surface of the CsI ( $\mathrm{Tl})$ crystal in order to absorb stray electrons that are produced in the subject. In this work we have proposed the use of a leaded glass plate to replace the Lucite ${ }^{\circledR}$ plate. Using leaded glass instead of Lucite $^{\circledR}$ has significant advantages like better optical coupling, ensuring a more firm and uniform adhesion of the two surfaces, and better absorption of brehmsstrahlung as well as electrons produced in the subject by the primary beam. In addition, we hypothesized that the leaded glass would act as a build up plate and, for an optimal thickness, would improve the QDE and DQE at the detector.

\subsection{Methods}

\subsubsection{Monte Carlo Simulations}

Monte Carlo simulations were performed using the ITS one dimensional TIGERP Monte Carlo computer program $^{46,47}$ to calculate the absorbed energy deposition (AED) or the pulse height spectrum for energy deposition in the CsI (Tl) crystal. The AED gives the number of energy deposition events in an energy bin of width $(E+\Delta E)$ as a function of energy E. Simulations were performed for 28 different thickness combinations of CsI (Tl) and lead equivalent of leaded glass 
radiator (see Table 3-1). The $6 \mathrm{MV}$ linac spectrum from Mohan et $\mathrm{al}^{48}$ was used as the input $\mathrm{x}$ ray spectrum, with $4 \times 10^{7}$ photon histories. The photons histories were divided into 40 batches (I million photons per batch). In order to model the system response to the input spectrum for various patient thicknesses, simulations were also performed with the $6 \mathrm{MV}$ spectrum attenuated by $10,20,30$ and $40 \mathrm{~cm}$ of water, for a $12.7 \mathrm{~mm}$ thick CsI (Tl) coupled to a $1.5 \mathrm{~mm}$ thick lead radiator.

Table 3-1. CsI (Tl) and lead thicknesses used for Monte Carlo simulations

\begin{tabular}{|c|c|}
\hline CsI (Tl) thickness (mm) & Lead thickness (mm) \\
\hline 3.0 & 0.0 \\
\hline 12.7 & 0.25 \\
\hline 20 & 0.5 \\
\hline 25 & 1.0 \\
\hline & 1.5 \\
\hline & 2.0 \\
\hline & 2.5 \\
\hline
\end{tabular}

\subsubsection{Calculation of QDE}

The QDE of the detector was calculated for all the different thickness combinations of CsI (Tl) and leaded glass. The QDE was calculated as the ratio of the total number of energy deposition events in the detector to the number of incident $x$-ray quanta (i.e. $4 \times 10^{7}$ ). QDE values were also obtained for a $12.7 \mathrm{~mm} \mathrm{CsI} \mathrm{(Tl)} \mathrm{-} 1.5 \mathrm{~mm}$ lead combination when the input X-ray spectrum was attenuated by $10,20,30$, and $40 \mathrm{~cm}$ of water.

\subsubsection{Calculation of DQE}




\subsubsection{DQE at the Detector}

The zero frequency DQE at the detector was calculated as the ratio of the squares of the output and input signal to noise ratios as shown in equation 3.3.3-1

$$
\operatorname{DQE}(0)=\frac{\mathrm{SNR}_{\text {out }}^{2}}{\mathrm{SNR}_{\text {in }}^{2}}
$$

The input signal to noise ratio was calculated as

$$
\mathrm{SNR}_{i n}=\frac{q_{\mathrm{A}}}{\sqrt{q_{\mathrm{A}}}}
$$

where $q_{A}$ is the total number of incident quanta, which is the input signal. The input noise is that due to quantum fluctuations ${ }^{45}$ i.e. the square root of the number of incident quanta. The output SNR at the detector was calculated by considering the SNR of individual energy bins. The signal in each energy bin $i$ was calculated as the number of energy deposition events in the bin $\left(N_{i}\right)$ times the average energy of the bin $\left(E_{i}\right)$. The noise in each bin was calculated as the square root of the number of events times the average energy. For $n$ energy bins, the total output SNR at the detector was then calculated as

$$
\mathrm{det}_{\mathrm{dNR}}=\frac{\sum_{i=1}^{n} N_{i} E_{i}}{\sqrt{\sum_{i=1}^{n} N_{i} E_{i}^{2}}}
$$




\subsubsection{DQE of the System}

The DQE for the complete system was also calculated based on equation (3.3.3.1-1). Calculations were performed for the Astromed ${ }^{\circledR}$ CCD based system and the PixelVision ${ }^{\circledR}$ CCD based system. The input SNR calculation was calculated using equation (3.3.3.1-2). The output SNR of the complete system was calculated by considering the effect of the secondary light quantum sink caused due to inefficient light collection by the lens and the CCD. It has been shown that in megavoltage radiographic imaging systems using a scintillating screen coupled to a detector with a lens, the output signal to noise ratio is reduced by a factor of $r,{ }^{49}$ where

$$
r=\left(1+\frac{1}{g}\right)^{\frac{1}{2}}
$$

The factor $g$ is the mean number of detected optical photons for each detected $\mathrm{x}$-ray photon and is given by

$$
g=g 1 . g 2 . g 3
$$

where $g 1$ is the mean number of optical photons produced per absorbed x-ray photon, $g 2$ is the probability that such a photon will reach the light detector, based on the geometrical optics and $g 3$ is the probability that the light photon will be transmitted by the optical elements and detected by the light detector.

The factor $g l$ is determined by considering the fact that $\mathrm{x}$-ray photons on an average, produce one light photon per $25 \mathrm{eV}$ of absorbed energy in a NaI (Tl) crystal. ${ }^{50}$ Since, a CsI (Tl) crystal has approximately $63 \%$ peak light output of a $\mathrm{NaI}(\mathrm{Tl})$ crystal, ${ }^{51}$ about $40 \mathrm{eV}$ is required to produce one light photon, following an x-ray interaction in the CsI (Tl) scintillator. The value of 
$g 2$, depends upon the index of refraction of the crystal, the $\mathrm{f}$ number of the lens and the magnification of the optical arrangement. Therefore

$$
g 2=\frac{1}{\left[4 \cdot n \cdot F \cdot\left(1+\frac{1}{m}\right)\right]^{2}}
$$

where $\mathrm{n}$ is the index of refraction for $\mathrm{CsI}(\mathrm{Tl})$ (equal to 1.79$), \mathrm{F}$ is the $\mathrm{f}$ number of the lens, equal to 0.95 and $\mathrm{m}$ is the magnification, equal to $1 / 34$ for the Astromed ${ }^{\circledR}$ based system and $1 / 13$ for

the PixelVision ${ }^{\circledR}$ based system. The factor $g 3$ is dominated by the light detection efficiency of the CCD. Therefore, $g 3$ was considered to be equal to the light collection efficiency of the CCD, i.e. 0.3 for the Astromed ${ }^{\circledR}$ CCD and 0.9 for the PixelVision ${ }^{\circledR}$ CCD.

From equation (3.3.3.2-1) and the definition of $g l$, it is clear that the value of $r$ for each energy bin is different due to differences in the mean number of optical photons produced in each energy bin. Therefore, in order to calculate the output SNR of the complete system, the $r$ factor was calculated for each bin and incorporated in the expression for the output signal to noise ratio.

$$
\mathrm{Syst}_{\mathrm{out}}=\frac{\sum_{i=1}^{n} N_{i} E_{i}}{\sqrt{\sum_{i=1}^{n} N_{i} E_{i}^{2} r_{i}^{2}}}
$$

\subsubsection{Calculations for Water Attenuated X-ray Spectra}


QDE and DQE calculations were performed as outlined in the above two sections, for a $12.7 \mathrm{~mm}$ thick CsI (Tl) crystal - $1.5 \mathrm{~mm}$ lead radiator combination with the input spectrum attenuated by $10,20,30$ and $40 \mathrm{~cm}$ of water. The attenuated spectra were calculated using the mass attenuation coefficients of water at the mid-energies of the energy bins used in the input spectrum. The mass attenuation coefficients at these energies were obtained by interpolating the values available from published data. ${ }^{33}$

\subsubsection{Calculation of DQE(f)}

The frequency dependent detective quantum efficiency, DQE(f), was calculated for a system using a $12.7 \mathrm{~mm}$ thick $\mathrm{CsI}(\mathrm{Tl})$ scintillator with $1.5 \mathrm{~mm}$ lead radiator. The frequency dependent DQE is given by ${ }^{45}$

$$
\operatorname{DQE}(f)=\mathrm{DQE}(0) \cdot \mathrm{MTF}^{2}(f)
$$

The MTF curve for the $12.7 \mathrm{~mm} \mathrm{CsI} \mathrm{(Tl)} \mathrm{was} \mathrm{obtained} \mathrm{earlier}{ }^{42}$ by imaging a $2 \mathrm{~mm}$ thick tungsten bar pattern with bars at 1.0, 1.3, 1.6, 1.8 and $2.0 \mathrm{cy} / \mathrm{mm}$.

Equation (3.3.3.4-1) assumes that the system noise power spectrum (NPS) is white i.e. the noise is independent of the spatial frequency. This assumption is valid since the input noise is the x-ray quantum noise determined by the Poisson statistics and the output noise is dominated by the fluctuation in the energy deposition due to absorption events in the crystal. Therefore, if the detector is large and uniform, the input and output noise can be considered to be independent of the spatial frequency. ${ }^{52}$ 


\subsection{Results}

Absorbed energy distributions (AEDs) were obtained for the 28 different thickness combinations of CsI (Tl) and lead equivalent of leaded glass. AEDs were also obtained for a $12.7 \mathrm{~mm}$ thick CsI (Tl) crystal coupled to a $1.5 \mathrm{~mm}$ thick lead radiator, using the water attenuated input spectra described in section 3.3.1. As a representative example, the AED for a $12.7 \mathrm{~mm}$ thick CsI (Tl) with $1.5 \mathrm{~mm}$ lead radiator is shown in figure 3-2. Due to the high number of photon histories used (40 million), the statistical errors in calculating the AEDs (using the ITS Monte Carlo software) were less than 3\% for all cases. However, the input spectrum had significant statistical errors, ${ }^{48}$ especially at lower energies, which may explain the high level of variance in the AED at low energies.

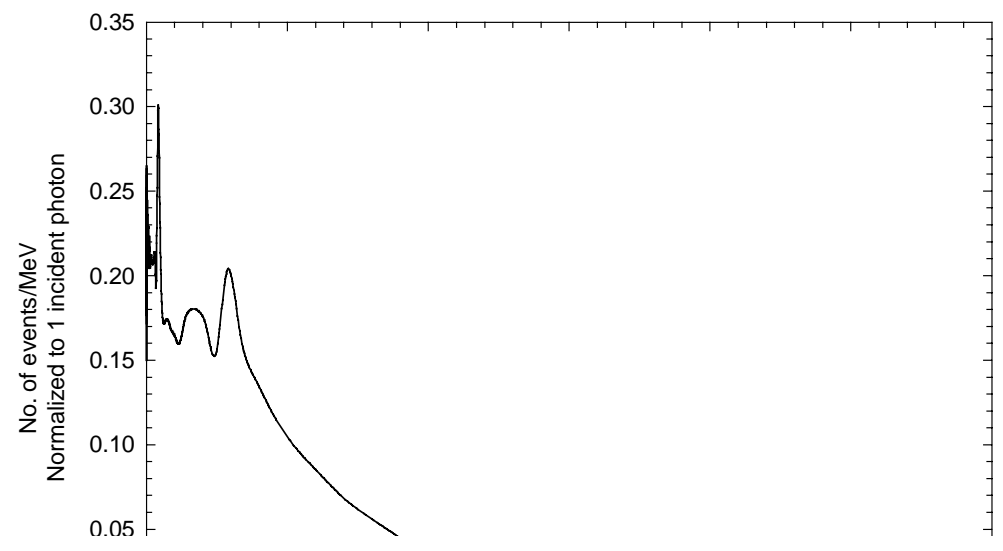

Figure 3-2. Absorbed energy distribution for $12.7 \mathrm{~mm} \mathrm{CsI} \mathrm{(Tl)} \mathrm{with} 1.5 \mathrm{~mm}$ lead radiator

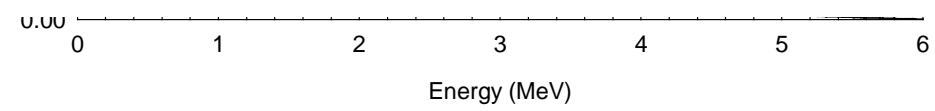

Figure 3-2. Absorbed energy distribution for $12.7 \mathrm{~mm} \mathrm{CsI} \mathrm{(Tl)} \mathrm{with} 1.5 \mathrm{~mm}$ lead radiator 
Figure 3-3 shows the QDE and DQE plots for all the 28 combinations of CsI (Tl) and lead. The zero frequency DQE is shown for a perfect light detector, for the present Astromed CCD based system with a $25 \mathrm{~mm}$ f 0.95 lens and for the proposed system which uses a Pixelvision $2048 \times$ 2048 pixel back surface thinned CCD coupled to a $50 \mathrm{~mm} \mathrm{f} 0.95$ lens. It can be seen that the DQE values for the proposed system lie only a few percent below those of the ideal light detector and are significantly higher than the values obtained using the present Astromed CCD based system. Although these calculations represent the upper limit of DQE that can be achieved (since radiation noise and readout noise are not considered), the results indicate that the proposed system could achieve very good performance. 

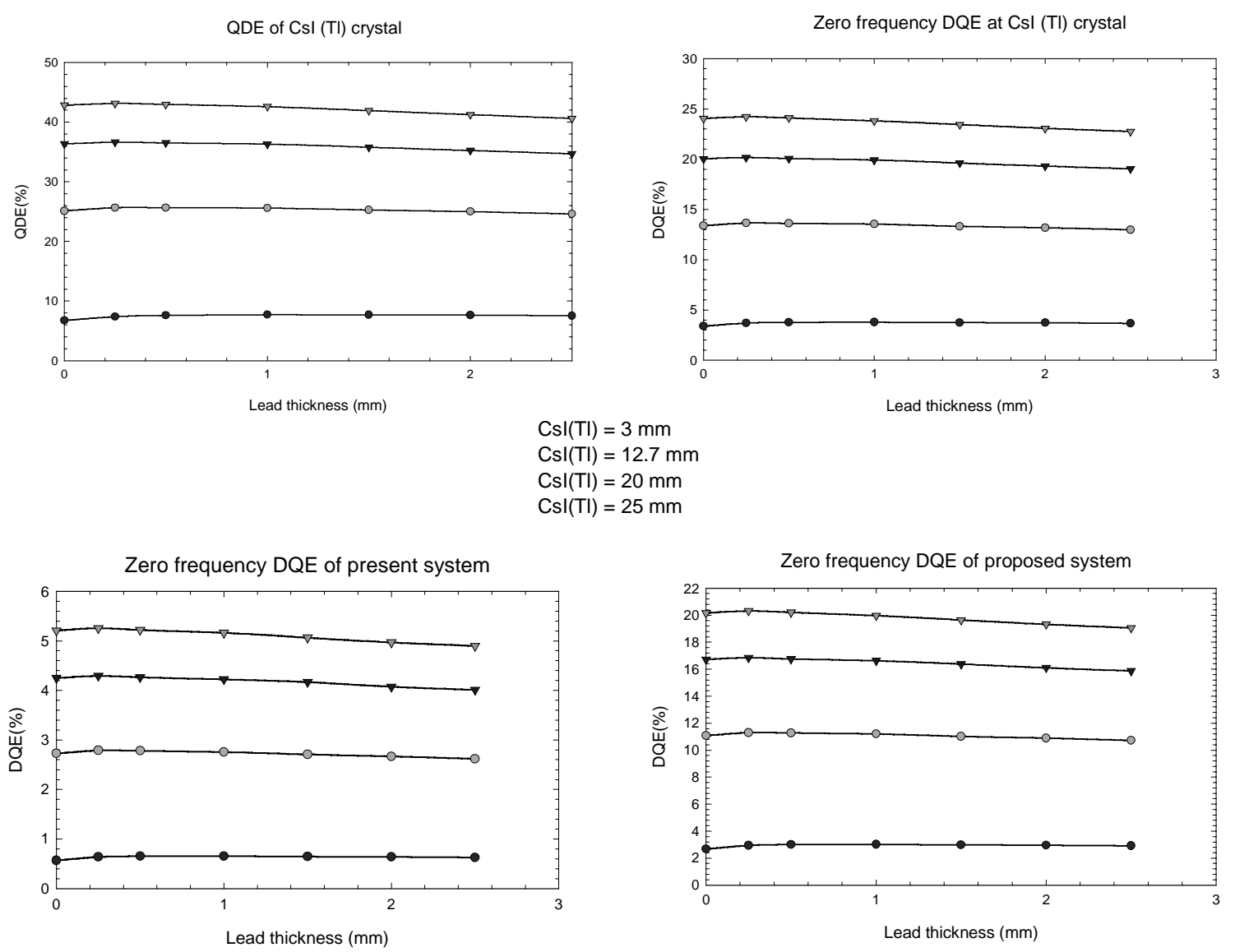

Figure 3-3. Theoretical QDE and DQE calculations for 28 thickness combinations of CsI (Tl) and lead equivalent of leaded glass. The DQE is shown for a perfect light detector, for the Astromed CCD based system and for the proposed Pixelvision back surface lit, thinned CCD based system.

From the plots in figure 3-3 it can be seen that the QDE and DQE remain relatively constant with lead thickness. There appears to be a slight initial increase in the QDE and DQE for lead thicknesses up to $0.5 \mathrm{~mm}$, followed by a gentle roll-off. Although this slight rise in the curves suggests that leaded glass, up to an optimal thickness, will increase the DQE it cannot be concluded that this increase will cause a significant improvement in the system performance. However, the fact that the DQE does not drop significantly with lead thickness, can be used to design the proposed system using any reasonable thickness of leaded glass in order to effectively 
absorb the brehmstrahlung and electrons generated in the patient. This greater absorption of stray radiation may significantly improve the SNR transfer characteristics of the system.

Figure 3-4 shows the variation in DQE and QDE with the thickness of water absorber for a 12.7 mm thick CsI (Tl) crystal and a $1.5 \mathrm{~mm}$ thick lead radiator. It can be seen that the QDE and DQE are relatively constant with water thickness, which indicates that the calculations for QDE and DQE (figure 3-3) performed with zero water thickness are adequate for comparing different thicknesses of CsI (Tl) and lead radiator.

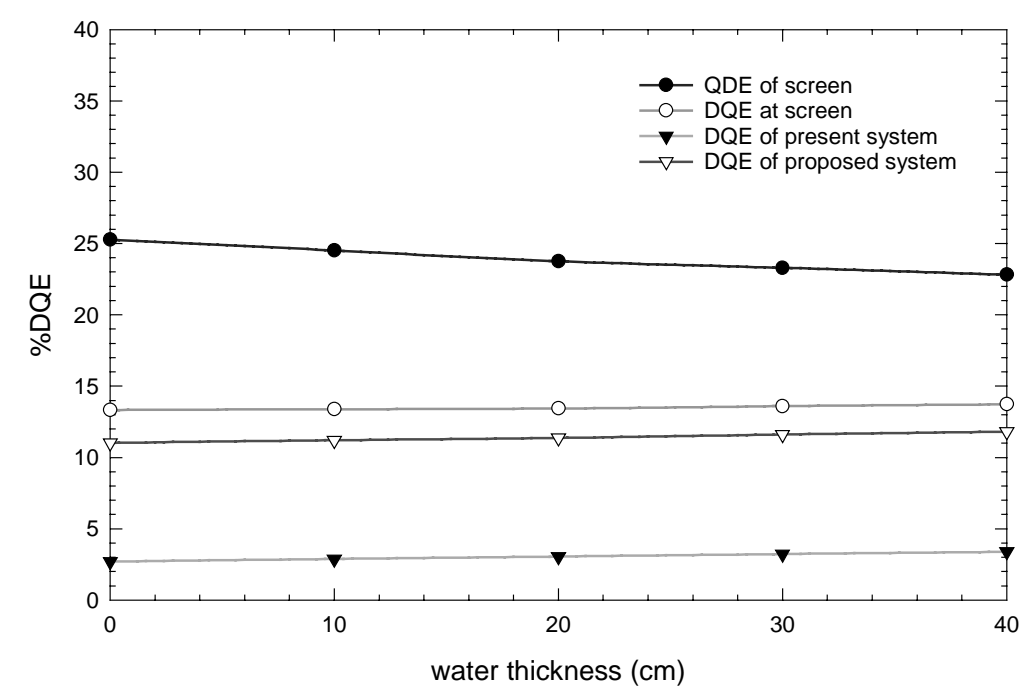

Figure 3-4. QDE and DQE of a $12.7 \mathrm{~mm} \mathrm{CsI} \mathrm{(Tl)} \mathrm{crystal} \mathrm{with} 1.5 \mathrm{~mm}$ lead radiator plotted against the thickness of water absorber. The DQE is shown for a perfect light detector, the present system and the proposed system.

The frequency dependent detective quantum efficiency DQE(f) for the proposed CsI (Tl) Pixelvision CCD system using a $12.7 \mathrm{~mm}$ CsI (Tl) screen with $1.5 \mathrm{~mm}$ lead equivalent of leaded 
glass is shown in figure 3-5. The DQE(f) of the CsI (Tl) - CCD system is compared with those of an amorphous silicon flat panel EPID and a TV camera - phosphor screen based EPID. The DQE(f)s of the flat panel EPID and the TV camera based EPID were scanned from published data $^{53}$ and re-plotted on the DQE(f) plot for the CsI (Tl) system. The DQE(f) of the amorphous silicon EPID had to be recalculated because the original calculation did not consider the finite pixel size of the flat panel array $(450 \mu \mathrm{m})$. The recalculation was performed by assuming half the pixel size (i.e. $225 \mu \mathrm{m}$ ) and multiplying the original $\mathrm{DQE}(\mathrm{f})$ by the square of the MTF of the pixel (see chapter II, equation 3.3.1-1). The increase in noise due to reduction in pixel size was

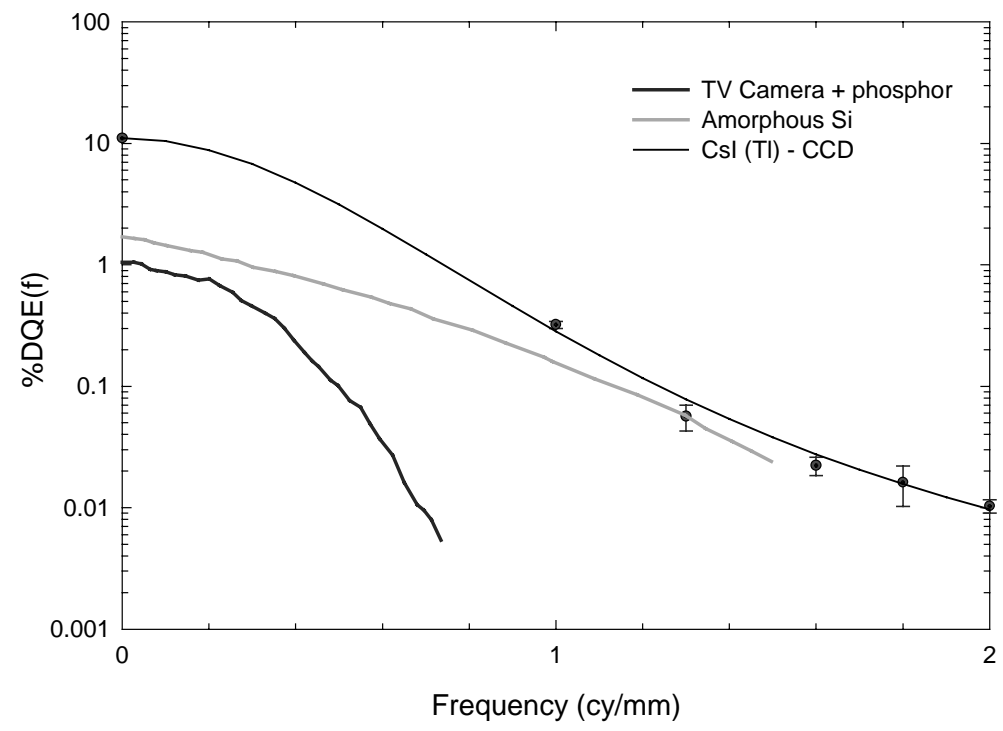

Figure 3-5. Comparison of DQE(f) of the proposed CsI (Tl) - CCD system, a TV camera based EPID and an amorphous silicon flat panel EPID.

neglected. It should also be noted that the DQE(f) calculation for the TV based and the flat panel systems assumes a white noise power spectrum (NPS), similar to the DQE(f) calculation for the 
CsI (Tl) system. This assumption of a white NPS for all three systems facilitates their direct comparison based on the DQE(f).

From figure 3-5 it can be seen that the proposed CsI (Tl) system performs significantly better compared to the amorphous silicon EPID over the range of frequencies up to $1.2 \mathrm{lp} / \mathrm{mm}$. This range contains most of the clinically useful information (e.g. large bony landmarks and structures) for determining placement errors. Both the CsI (Tl) and the amorphous silicon systems perform roughly equally well for higher frequencies. The TV camera - phosphor screen based EPID performs significantly worse compared to the other two systems over the entire frequency range.

\subsection{Conclusions}

In this work, the theoretically predicted performance of a CsI (Tl) - CCD based portal imaging system was analyzed by determining performance quality indices like QDE, DQE and DQE(f). The DQE was found to be relatively constant with lead thickness, which suggests that any reasonable thickness of leaded glass can be coupled to the $\mathrm{CsI}(\mathrm{Tl})$ screen in order to absorb stray electrons and brehmstrahlung generated inside the patient. This greater absorption of scattered radiation could siginificantly improve the SNR transfer efficiency of the system.

Although the analysis indicates the upper limits of performance that can be achieved, it is clear from the results that a practical electronic portal imaging system based on a thick transparent CsI (Tl) scintillator coupled to a cooled CCD camera is feasible. From figure 3-5 it can be seen that the use of a thick transparent scintillator with a high quality CCD camera can greatly improve the 
performance of TV based EPIDs due to the order of magnitude higher QDE of the CsI (Tl) screen. The comparison of the proposed system with an amorphous silicon based EPID suggests that the proposed CsI ( $\mathrm{Tl}$ ) based system can perform significantly better at lower frequencies (which contain most of the clinically useful information), while performing roughly equally well at higher frequencies. 


\section{CHAPTER 4}

\section{System Design}

\subsection{Basic Design}

The basic design of the CCD - CsI (Tl) portal imaging system ${ }^{41}$ is shown in figure 4-1. Briefly, the system consists of a $12.7 \mathrm{~mm}$ thick, $20 \mathrm{~cm}$ diameter CsI (Tl) scintillator crystal that acts as the x-ray detector. The scintillator is coupled to an Astromed ${ }^{\circledR}$ cooled CCD camera using a high speed lens. The entire imaging apparatus is kept in a light tight box (shown in figure 4-2a) to prevent extraneous light from falling on the CCD camera. The CsI (Tl) screen is shown in figure $4-2 b$.

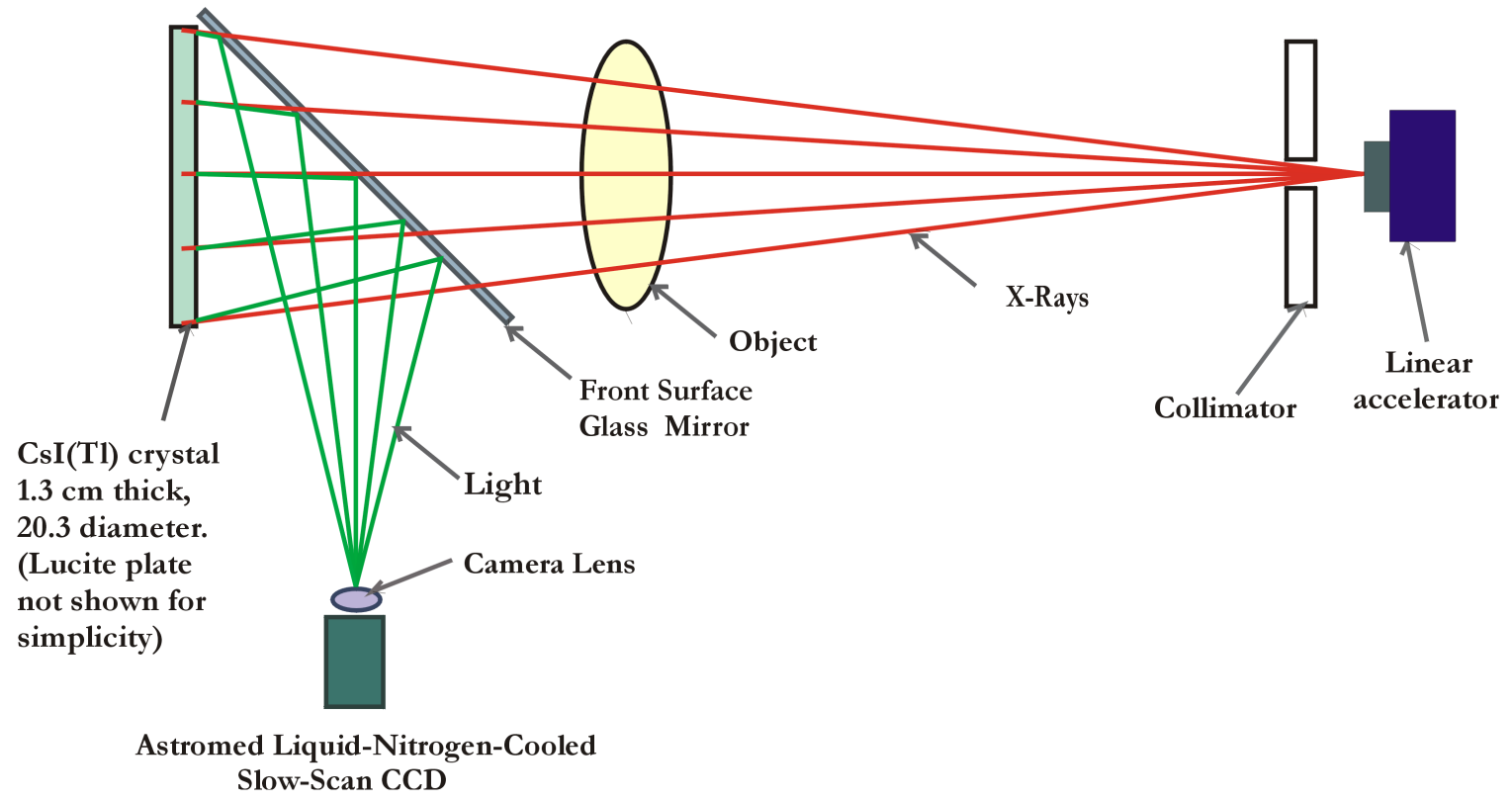

Figure 4-1. Experimental arrangement for the CCD-CsI (Tl) based portal imaging system. 


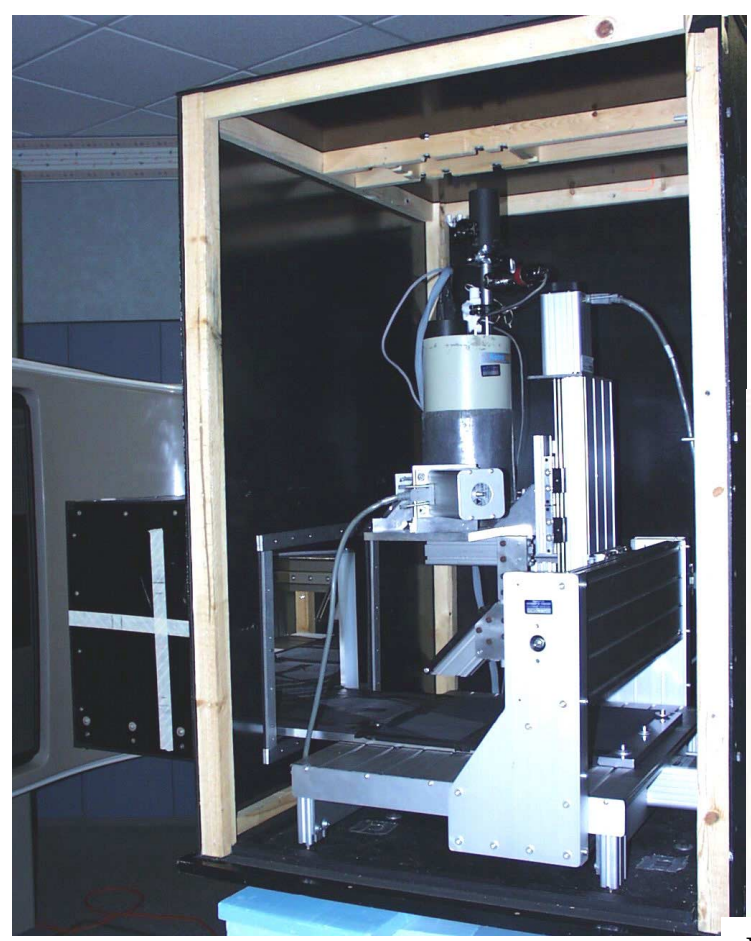

Figure 4-2.a CCD - CsI (Tl) portal imager assembly

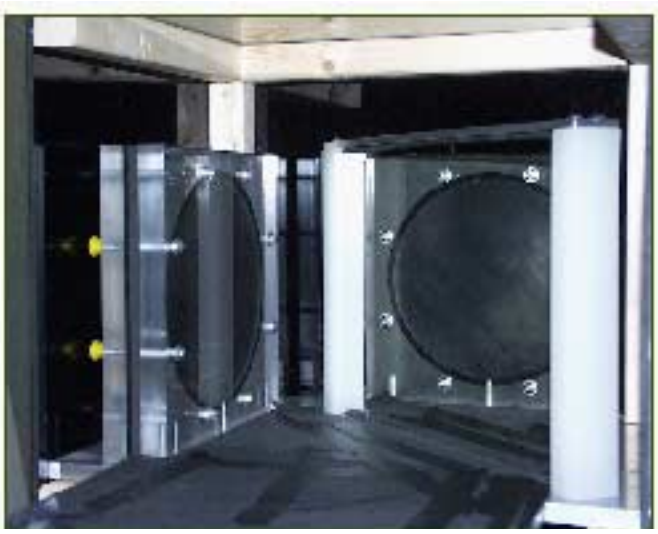

Figure 4-2.b Photograph of the CsI (Tl) screen. Note that the reflection of the screen in the mirror, appears completely black indicating the insensitivity of the system to mirror glare

\subsection{Design Considerations}

\subsubsection{Geometry}

The system geometry is designed to maximize the spatial resolution. High spatial resolution is accomplished by having a mirror at 45 degrees on the input side of the system, to reflect the light coming from the CsI (Tl) crystal onto the CCD camera. Since the mirror is on the input side, the camera sees light rays which if projected backwards would lie along the paths of the x-rays in the scintillator. The distance between the CCD and the CsI ( Tl) screen is made equal to the distance between the x-ray focal spot and the CsI (Tl) screen, divided by the index of refraction of CsI (Tl) (equal to 1.79). 


\subsubsection{Crystal - Machining of Surface and Optical Coating}

The CsI (Tl) crystal is machined on both surfaces. Machining of the surfaces, rather than polishing, ensures that there is minimal total internal reflection of light rays produced within the crystal. The front surface of the crystal is coupled to a Lucite plate using an optical coupling compound. The coupling compound provides an optically flat surface for the light rays that exit from the CsI (Tl) crystal and are sensed by the CCD camera. The back surface of the CsI (Tl) crystal is coated with black epoxy resin in order to absorb light rays scattered inside the crystal.

\subsubsection{Lucite/Leaded Glass Absorber/Radiator}

A $25 \mathrm{~mm}$ thick Lucite plate (not shown in figure4-1) is coupled to the front surface of the CsI (Tl) crystal. The Lucite plate absorbs stray electrons that are produced in the subject.

In the proposed system we plan to use a leaded glass plate in place of the Lucite plate. Using leaded glass instead of Lucite will have significant advantages like better optical coupling and absorption of brehmstrahlung as well as electrons produced in the subject by the primary beam. The leaded glass may also act as a radiator or a build up plate and serve to increase the QDE of the detector.

\subsubsection{Choice of Lens}

The main factors taken into consideration while selecting a proper lens for the CCD camera are the light collection efficiency and the depth of field. 


\subsubsection{Light Collection Efficiency}

Since TV based portal imaging systems are light quantum limited, it is essential to use a high speed lens in order to maximize the light collection efficiency. The speed of a lens is denoted by its $f$ number which is the ratio of the focal length to the diameter of the entrance pupil. Thus a high speed lens will have a low $\mathrm{f}$ number (e.g. 0.95) while a lower speed lens will have a higher $\mathrm{f}$ number (e.g. 2.8).

\subsubsection{Depth of Field}

Depth of field is the range of object distances for a given image distance, throughout which the image appears sharp. For the CsI (Tl) - CCD EPID, a relatively large depth of field is desirable since it allows the use of a scintillator of any reasonable thickness (for higher QDE) without significant loss of resolution due to focussing problems. Depth of field depends upon the $f$ number and the focal length of the lens. The formulas for depth of field are derived assuming that for conjugate object and image distances, the lens produces a sharp point image of a point source object and if the object distance is changed from the ideal conjugate relationship, the image becomes a circular blur called the circle of confusion. ${ }^{54}$ Obviously, for a sharper image, the circle of confusion should be as small as possible.

Consider a lens focussed sharply on an object plane $\mathrm{O}$ at a distance $\mathrm{s}$ from the principal plane of a lens. Let $\mathrm{z} 1$ and $\mathrm{z} 2$ be the distances of planes 1 and 2 respectively from $\mathrm{O}$ as shown in figure 43. Let $\mathrm{D}$ be the diameter of the entrance pupil. Rays from a point object lying on plane 1 and passing through the entrance pupil, intersect plane $\mathrm{O}$ to form a 
circular disc of diameter c. Since the plane O is in sharp focus, the rays from this "disc object" form a disc image of diameter $c^{\prime}$. Similarly, a point object in plane 2 forms a disc image of a disc object in plane $\mathrm{O}$. The diameter of this disc object is known as the circle of confusion.

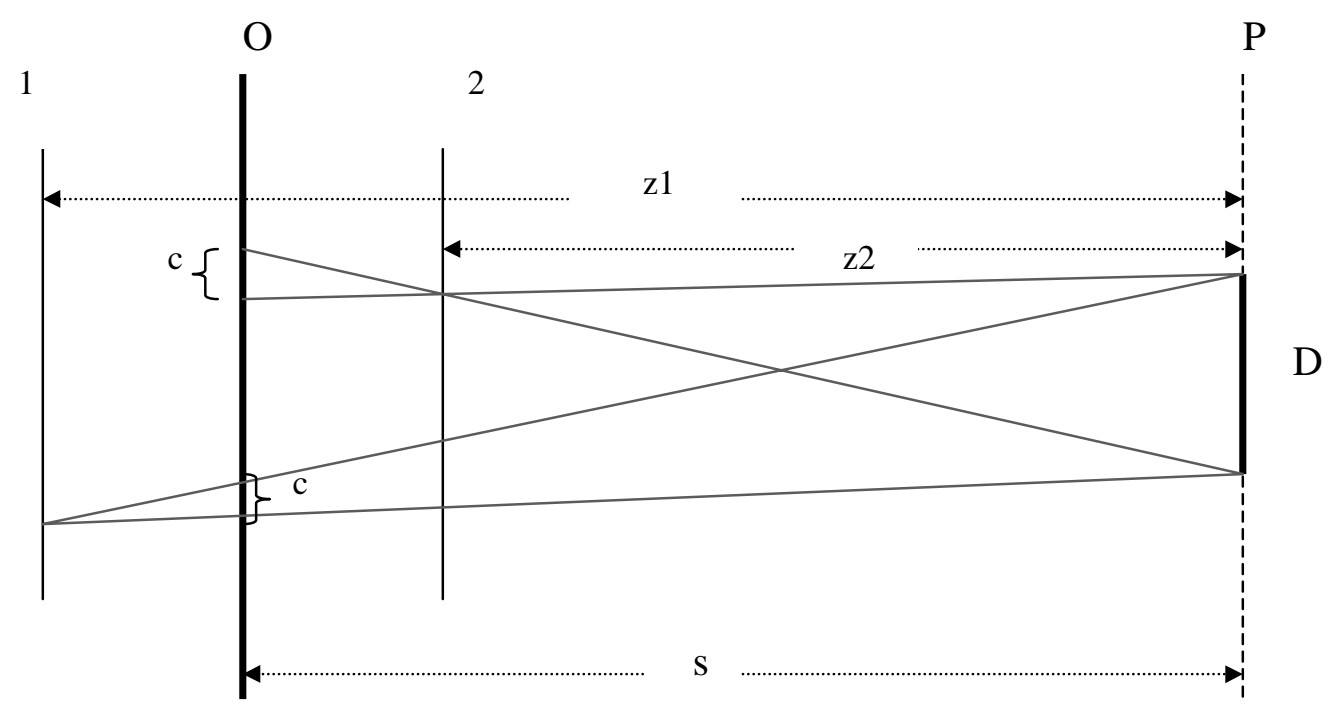

Figure 4-3. Depth of field for a photographic lens

For a photographic lens, we can assume that the distance between the entrance pupil and the principal plane is negligible compared to the object distance. Also, if the object is considered to be at infinity, the image is formed at a distance equal to the focal length f. From the geometry shown in figure 2-1, the following relations can be obtained. ${ }^{54}$

$$
\begin{aligned}
& z_{1}=\frac{s . f^{2}}{f^{2}-c^{\prime} \cdot N \cdot s} \\
& z_{2}=\frac{s . f^{2}}{f^{2}+c^{\prime} \cdot N . s} 62
\end{aligned}
$$


where $\mathrm{N}$ is the f number of the lens. The depth of field can then be calculated as

$$
z_{1}-z_{2}=\frac{2 s^{2} \cdot f^{2} \cdot c^{\prime} \cdot N}{f^{4}-\left(c^{\prime} \cdot N \cdot s\right)^{2}}
$$

For a CCD camera, the diameter of the circle of confusion in the image plane $\left(c^{\prime}\right)$, is related to the pixel size of the CCD i.e. it is the diameter of the circle that is inscribed inside a pixel. Thus, equation 2.4.2-3 can be used to select an appropriate lens for a required depth of field. For very small pixel sizes i.e. $c^{\prime} \rightarrow 0$, equation 2.4.2-3 can be approximated as

$$
z_{1}-z_{2}=\frac{2 s^{2} \cdot c^{\prime} \cdot N}{f^{2}}
$$

Thus, we can see that the depth of field is approximately proportional to the $\mathrm{f}$ number of the lens and inversely proportional to the square of the focal length. Table 4-1 shows the theoretically calculated values of the depth of field (i.e. using equation 2.4.2-4) using different lenses and $\mathrm{f}$ numbers, for the present Astromed CCD based system (pixel size $=22$ microns) and for the proposed PixelVision CCD based system (pixel size = 15 microns). 
Table 4-1. Depth of field (DOF) obtained using lenses with different focal length and f numbers. DOF values are shown for the Astromed CCD and PixelVision CCD based systems

\begin{tabular}{|c|c|c|c|}
\hline $\begin{array}{c}\text { Focal Length } \\
(\mathbf{m m})\end{array}$ & \multicolumn{3}{|c|}{ Depth of Field (cm) } \\
\hline \multicolumn{3}{|c|}{ Astromed Camera (pixel size = 22 microns) } \\
\hline 25 & F 0.95 & F 1.0 & F 2.8 \\
\hline 50 & 4.83208 & 5.0864 & 14.24192 \\
\hline 100 & 1.20802 & 1.2716 & 3.56048 \\
\hline \multicolumn{3}{|c|}{0.3179} & 0.89012 \\
\hline 25 & 0.302005 & 0.21675 & 0.6069 \\
\hline 50 & PixelVision Camera (pixel size =15 microns) \\
\hline 100 & 3.2946 & 3.468 & 9.7104 \\
\hline
\end{tabular}

\subsubsection{Astromed CCD Camera ${ }^{55}$}

The Astromed CCD camera is a liquid Nitrogen cooled 16 bit digital camera, having a $578 \times 416$ pixel array, with a pixel size of 22 microns. Of the 416 columns, 16 colums on either side are not exposed to light and are used for calibration of the detector. Each row of the CCD array is organized as a parallel register. The charge collected in each row is transferred row by row to the serial read out register. Typically, an entire scan takes about 6.0 seconds. The output of the serial register is given to the analog processing stage consisting of a low noise DC coupled differential pre-amplifier, an adjustable gain amplifier, an inverter and finally an integrator. The analog signal is then given to a 16 bit A/D converter which provides a serial digital output that can be processed by the RISC processor installed in the computer.

The Astromed CCD has a peak sensitivity to light of wavelength $700 \mathrm{~nm}$ (red light). The sensitivity of the camera to the peak emission of the CsI (Tl) crystal $(550 \mathrm{~nm})$ is approximately 30\%. The readout noise and SNR for the camera are approximately $46.9 \pm 0.5$ electrons and 695 respectively. ${ }^{37}$ 


\subsection{Noise: Sources and Removal}

Various sources of noise have been identified and analyzed for digital radiography systems. The noise in the CsI (Tl) - CCD portal imaging system can be broadly classified as systematic and random.

\subsubsection{Systematic Noise}

Systematic or fixed pattern noise is constant and temporally invariant. For the CCD - CsI (Tl) EPID the sources of systematic noise are

a) Aberrations in the CsI (Tl) crystal, lens, mirror and CCD

b) Non-uniformity of x-ray beam

c) Inconsistency of optical coupling

Systematic noise is removed during image processing, at the preprocessing stage, using a technique known as flat fielding, described in Chapter 5II, section 2.

\subsubsection{Random Noise}

The various sources of random noise in the CCD - CsI (Tl) system are

\subsubsection{X-ray quantum noise}

$\mathrm{X}$-ray quantum noise is caused by the fluctuation in the counting statistics of the detected x-ray quanta. The quantum noise can be reduced by increasing the number of detected quanta i.e. increasing the QDE of the detector. The only other way to reduce quantum noise is to increase the dose and thus increase the number of photons for a given detector. 


\subsubsection{Noise in the CCD Camera - Dark Current, Shot Noise and Read Out Noise}

The CCD camera is affected by noise in the form of dark current and shot noise. The origin of dark current has been explained in Chapter 2, section 2.3.2.2. For the Astromed CCD, which is liquid nitrogen cooled, the dark current is negligible i.e. of the order of 1 electron per pixel per hour.

Shot noise is caused due to the uncertainty in counting the photoelectrons collected by the CCD. Since one photoelectron is produced in the CCD per detected light photon, shot noise is the same as light quantum noise, which constitutes the secondary quantum sink for TV camera based portal imaging systems. As explained in chapter I, TV camera based portal imaging systems are light quantum limited and hence shot noise is the performance determinant of the system.

The emission of photons by a source is a Poisson process and likewise the photoelectrons collected by the CCD follow a Poisson distribution, with an uncertainty equal to the square root of the mean number of photoelectrons. Thus, the effect of shot noise in the CCD becomes serious in cases of low light intensity or low light collection efficiency.

The thermal noise due to the CCD readout electronics also contributes significantly to the overall noise level. There are various circuits in the readout electronics along with signal processing firmware, designed to minimize the effect of thermal noise in the Astromed CCD imaging system. ${ }^{55}$ Nevertheless a base read out noise level exists depending on the application. As explained in section 2.4.3, the read out noise for radiographic applications is $46.9 \pm 0.5$ electrons. 


\subsubsection{Impulse noise due to radiation hitting the CCD camera}

The CCD is sensitive to $\mathrm{x}$-ray photons as well as light photons. Stray $\mathrm{x}$-ray photons interact with the CCD to produce impulse noise. Impulse noise corrupts individual pixels, giving the final image a "salt and pepper" appearance. Adequate shielding of the CCD camera is required to significantly reduce the effect of impulse noise. ${ }^{44}$ In the prototype CsI (Tl) - CCD portal imaging system, the Astromed camera is protected from stray radiation using a $3 \mathrm{~mm}$ lead housing. However, this lead shielding was originally designed for diagnostic radiography applications. To stop high energy photons, the thickness of the lead housing would have to be of the order of 3 $\mathrm{cm} .{ }^{44}$ Therefore, in the present system, some radiation does interact with the CCD, corrupting a few pixels. The corrupt pixels have intensity values significantly higher compared to the normal pixels. The percentage of pixels that are corrupted by impulse noise can be easily determined by interactively setting a threshold and computing the number of pixels that lie above this threshold. It was found that in most cases that impulse noise corrupted approximately $8-10 \%$ of the pixels. This residual impulse noise is removed using image processing techniques like thresholded median filtering and adaptive median filtering, as explained in Chapter 7. 


\section{CHAPTER 5}

\section{Experimental Evaluation}

\subsection{Bases for Experimental Evaluation}

The performance of the CsI (Tl) - CCD portal imaging system was evaluated by determining the spatial resolution, depth of focus and contrast resolution. In addition, experiments were performed to determine the effect of phenomena like light glare and mirror glare, which are specific to TV based EPIDs.

\subsection{Spatial Resolution}

The spatial resolution of the CsI ( $\mathrm{Tl}$ ) - CCD portal imaging system was determined by imaging a resolution bar pattern made of $2 \mathrm{~mm}$ thick tungsten that had slots cut into it using Electro Discharge Machining (EDM). The bar pattern had bar groupings ranging from $0.1 \mathrm{lp} / \mathrm{mm}$ to 2.5 $\mathrm{lp} / \mathrm{mm}$. For this study, the bars at 1.0, 1.3, 1.6, 1.8 and $2.0 \mathrm{lp} / \mathrm{mm}$ were used. The lower frequency bars could not be used because they were not adequately visible in the limited field of view of the system. The bars at $2.5 \mathrm{lp} / \mathrm{mm}$ had been improperly machined and therefore could not be resolved in the images. The images were acquired using a Nikkor 100mm, f 2.8 Type E photo lens with a 1 diopter close up lens. This lens had a demagnification factor of 8.35 for a pixel size of $0.18 \mathrm{~mm}$ at the screen (located $85 \mathrm{~cm}$ from the lens) and a Nyquist limit of 2.7 $\mathrm{lp} / \mathrm{mm}$ at the screen and $3.3 \mathrm{lp} / \mathrm{mm}$ at the object.

A contrast enhanced image of the bar pattern is shown along with the associated modulation plot, in figure 5-1. The image was obtained by averaging 5 images, each taken at 20 monitor units (1 
monitor unit $\approx 1 \mathrm{cGy}$ ). The modulation curves were normalized by considering the signal through tungsten as $0 \%$ and signal through air as 100\%. The modulation transfer function (MTF) was calculated by averaging the differences in consecutive upper and lower peaks for each bar grouping.
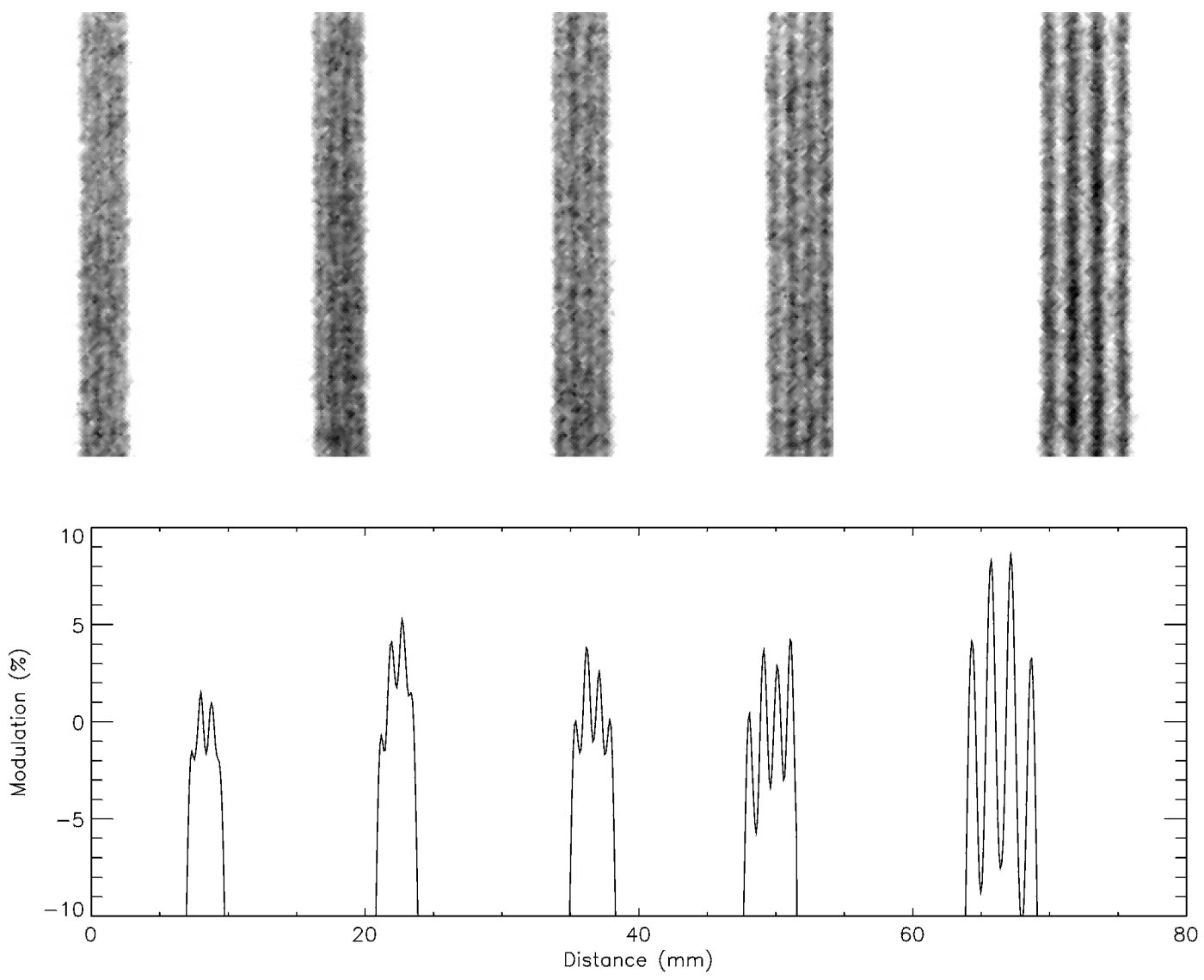

Figure 5-1. Bar pattern and modulation plot

The standard error (i.e. standard deviation of the means) for each spatial frequency was calculated as 


$$
\sigma_{\bar{x}}=\sqrt{\frac{\sum_{i=1}^{n}\left(x_{i}-\bar{x}\right)}{n(n-1)}}
$$

A fourth order polynomial was fitted to the logarithmic data. The polynomial coefficients were obtained by importing the data into Table Curve 2-D ${ }^{\circledR}$ and obtaining a polynomial least squares fit. The fit was chosen such that the curve approaches the theoretical limit of zero at twice the Nyquist frequency (i.e. $6.6 \mathrm{cy} / \mathrm{mm}$ ). The MTF curve is shown in figure 5-2.

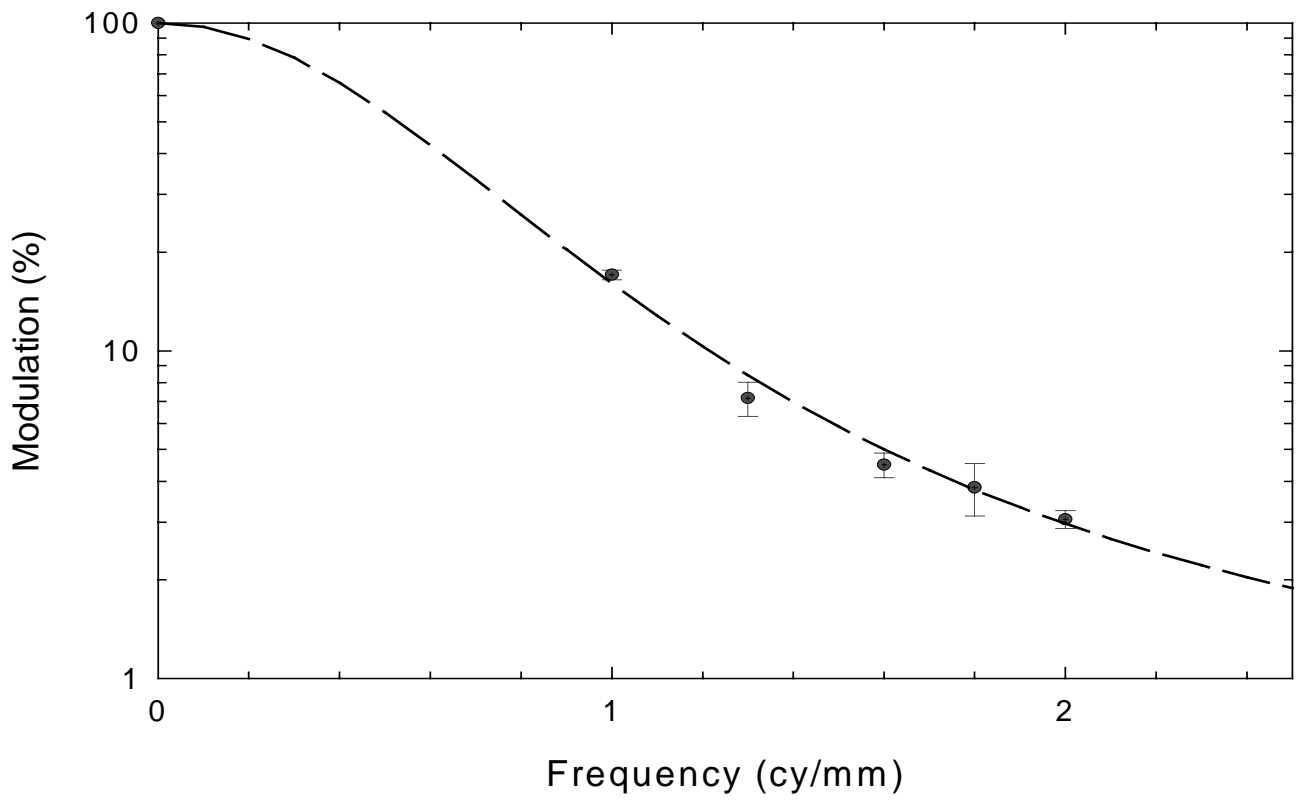

Figure 5-2. Modulation transfer function for CsI (Tl) - CCD EPID 
From the figure it can be seen that the CsI (Tl) - CCD portal imaging system has an MTF of over $3 \%$ at $2 \mathrm{lp} / \mathrm{mm}$, which is comparable to the resolution of portal films. ${ }^{13}$

\subsection{Depth of Field}

The concept of depth of field (DOF) was explained in chapter IV, section 2.4.2. Depth of field is an important design issue for TV based EPID using a thick transparent scintillator. The DOF limits the thickness of the detector that can be used. If the thickness of the scintillator is greater than the DOF, the spatial resolution will be degraded significantly. To determine the loss in spatial resolution due to the finite depth of field, the change in MTF was measured as a function of the distance between the lens and the CsI (Tl) screen for a constant distance between the lens and the CCD. The Nikkor 100mm, f2.8 lens (described in section 2) was used for this measurement. The camera and lens were mounted on a stepper motor controlled jig whose position was verified using a micro-switch. The variation of MTF for frequencies 1.0, 1.3 and 1.6 $\mathrm{lp} / \mathrm{mm}$ with respect to lens position is shown in figure 5-3. The MTF curves were obtained by fitting a symmetric $2^{\text {nd }}$ order polynomial to each data set (i.e. corresponding to each frequency). The polynomial coefficients were obtained using Table Curve 2-D, similar to the coefficients for the MTF curves in section 2. From figure 5-3, it can be seen that the MTF falls by less than $25 \%$ of the peak value over a distance of $\pm 1.5 \mathrm{~cm}$.

As shown in table 4-1 chapter IV, the Nikkor $100 \mathrm{~mm}$, f 2.8 lens coupled to the Astromed CCD camera (pixel size $0.022 \mathrm{~mm}$ ) gives a theoretical DOF approximately equal to $0.9 \mathrm{~cm}$. In order to get the same DOF for the proposed PixelVision based system, a $50 \mathrm{~mm}$, f1.0 lens would be required. From the DOF values shown in table 4-1, it is clear that in order have adequate DOF as 
well as high light collection efficiency, it is necessary to use a lens with a short focal length and a relatively small f number. For example, for the Astromed based system, a $50 \mathrm{~mm}$, f 1.0 lens could give a DOF of approximately $1.27 \mathrm{~cm}$.

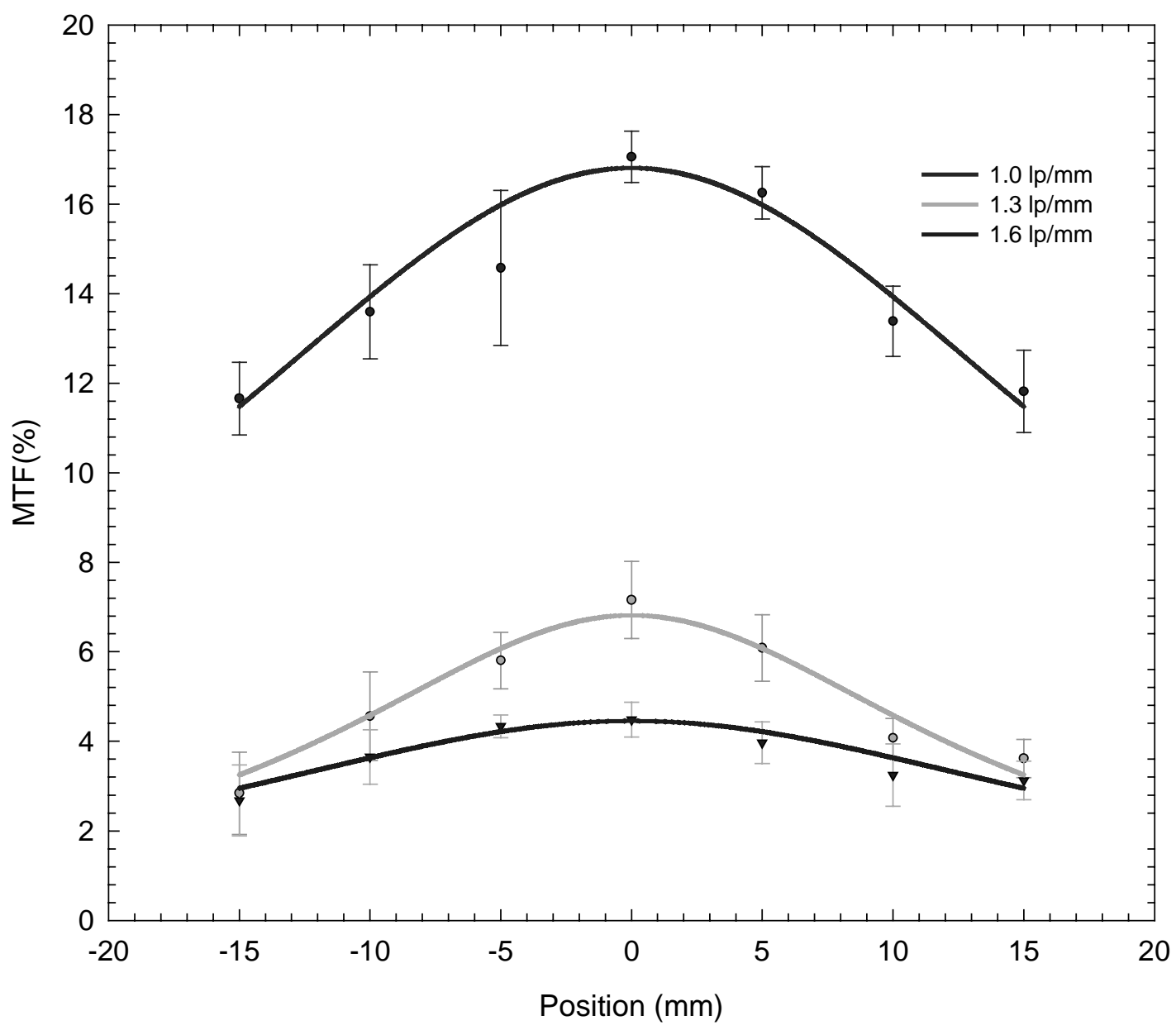

Figure 5-3. Variation in MTF with distance between lens and CsI (Tl) screen. 


\subsection{Contrast Resolution}

The contrast resolution of the system was determined qualitatively by imaging a low contrast phantom. The low contrast phantom consisted of a $5 \mathrm{~cm}$ thick Lucite slab with $10 \mathrm{~mm}$ diameter holes 2, 3, 4, 4.5, 5, and $6 \mathrm{~mm}$ deep, and $2 \mathrm{~mm}$ diameter holes 2, 3, 4, 4.5, 4.5 and $5 \mathrm{~mm}$ deep. The phantom was imaged in air and with $20 \mathrm{~cm}$ of added solid water $(10 \mathrm{~cm}$ on each side). The images of the low contrast phantom are shown in figure 5-4. From the figure it can be seen that all the low contrast objects are visible in all four images.

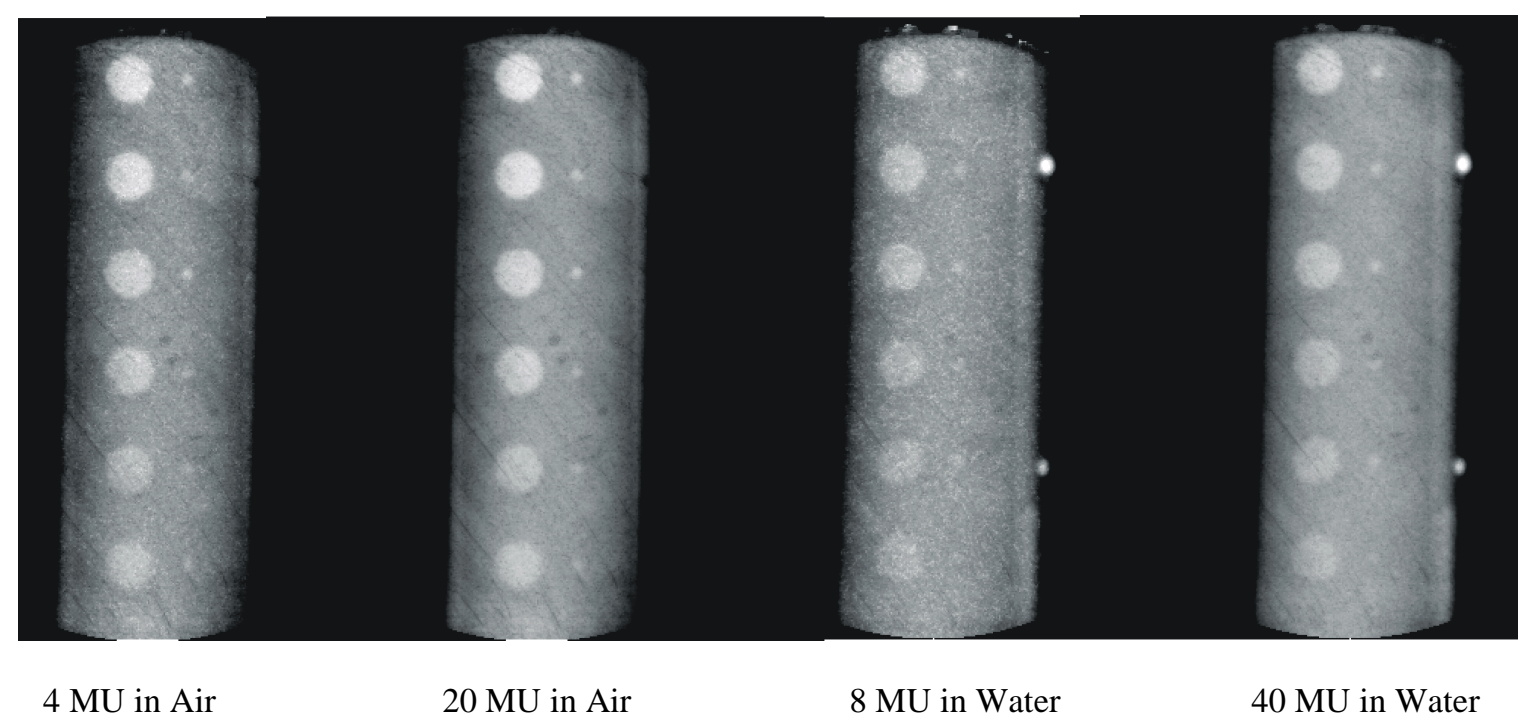

Figure 5-4. CCD-CsI (Tl) images of low contrast phantom, $5 \mathrm{~cm}$ thick Lucite with $10 \mathrm{~mm}$ diameter holes 2, 3, 4, 4.5, 5, and $6 \mathrm{~mm}$ deep, and $2 \mathrm{~mm}$ diameter holes 2, 3, 4, 4.5, 4.5 and $5 \mathrm{~mm}$ deep. The phantom is shown in air and with $20 \mathrm{~cm}$ of added solid water.

The contrast resolution of the CsI (Tl) - CCD EPID was compared with high contrast Kodak ECL film and a commercial electronic portal imaging system, Philips SRI - 100. Figure 5-5 shows images of the low contrast phantom acquired using the above three modalities. The images were taken at comparable doses, with the phantom and $20 \mathrm{~cm}$ of solid water. From figure 
5-5, it can be seen that the contrast resolution of the CsI ( Tl) - CCD system is comparable to ECL film, and significantly superior to the Philips SRI - 100 EPID.

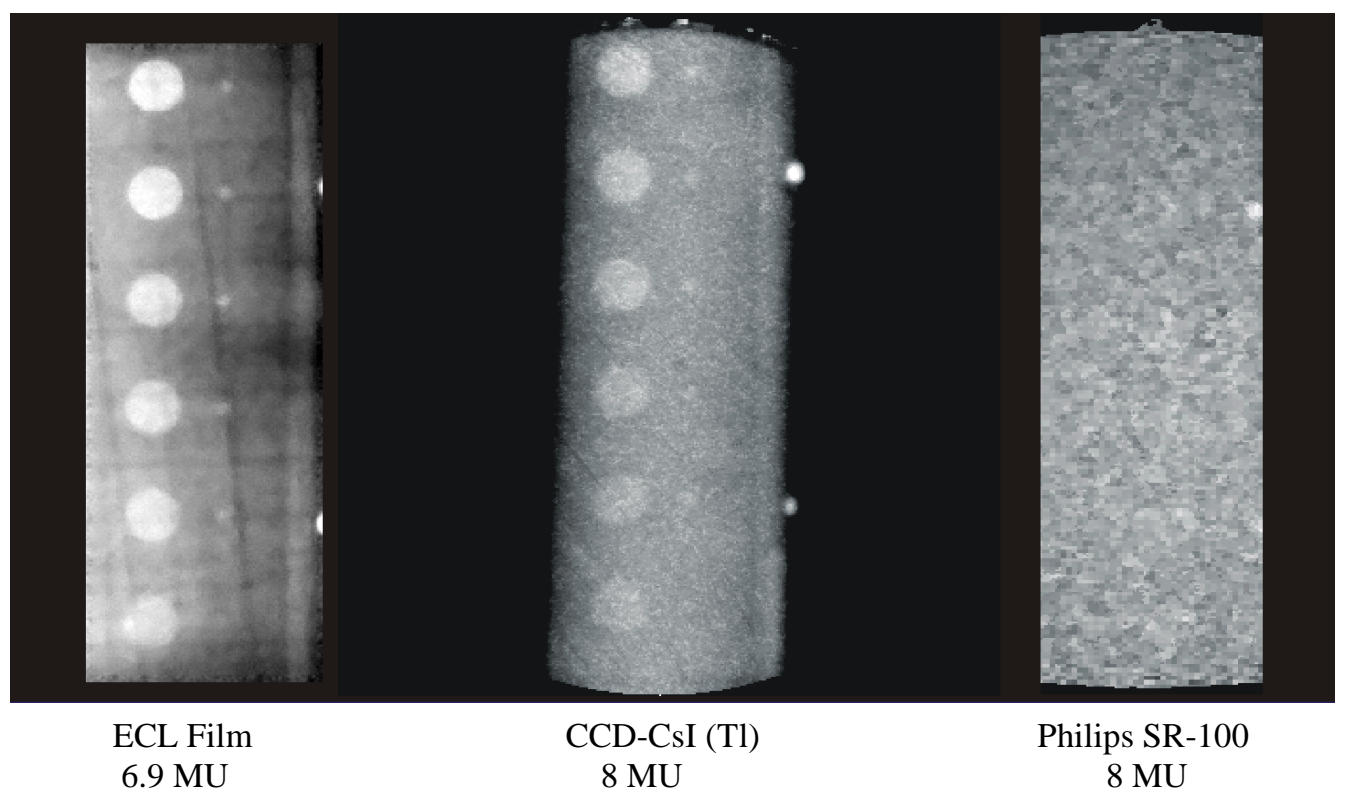

Figure 5-5. Comparison of contrast resolution. Low contrast phantom with $20 \mathrm{~cm}$ of solid water

\subsection{Light Scatter in the CsI (Tl) Crystal}

The optical glare due to light rays scattered and totally internally reflected inside the CsI (Tl) crystal was measured. Ordinarily, such scattered light would produce a significant amount of optical glare in a thick clear scintillator with polished, optically flat surfaces. To minimize the effect of scattered light in the prototype CsI (Tl) - CCD system, the surfaces of the CsI (Tl) were rough machined and the back and side surfaces were coated with optically absorbent black paint as shown in figure 5-6. This design ensures that the light rays are scattered at random angles (avoiding multiple total internal reflections) and are absorbed by the black paint. 


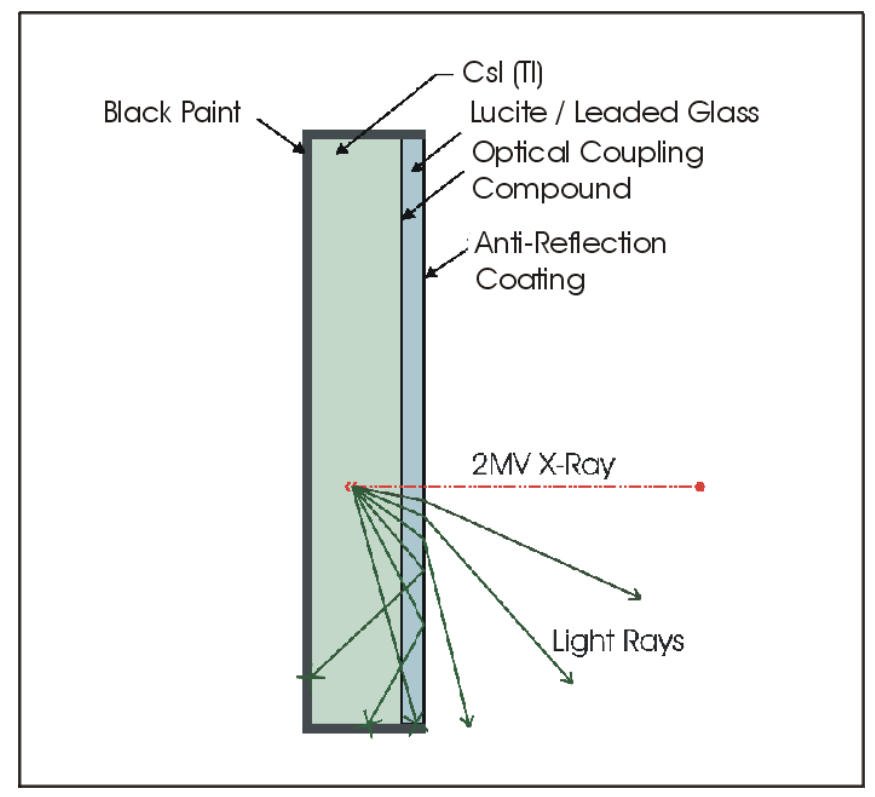

Figure 5-6. Absorption of scattered light inside the CsI (Tl) crystal.

To measure the scattering of light inside the CsI (Tl) crystal, a $6 \mathrm{MVp}$ x-ray beam produced by a Siemens MD2 linear accelerator was used. The beam was highly collimated $(2 \mathrm{~mm} \times 3 \mathrm{~mm})$ by adjusting the collimator jaws. An additional lead collimator, $20 \mathrm{~cm}$ thick and $1 \mathrm{~cm}$ in diameter was used to absorb the radiation produced in the accelerator collimator jaws. The beam was made incident at six different positions on the CsI (Tl) crystal, going from side to side, approximately along a diameter. To compensate for scattered radiation from the head of the accelerator, two images were taken at each position, one image with the collimated beam reaching the $\mathrm{CsI}(\mathrm{Tl})$ crystal (image 1 ) and the other image with the $\mathrm{x}$-ray beam blocked by a 20 $\mathrm{cm}$ thick lead block (image 2). Image 2 was subtracted from image 1 in order to obtain a difference image, which consisted of the signals due to the x-ray beam and scattered light. 
The scattered light signal was smoothed using a gaussian kernel to remove false peaks due to random noise. The signal due to the primary $\mathrm{x}$-ray beam was retained unchanged. The surface plots of the glare patterns at the six different positions are shown in figure 5-7. The z-axis shows the percent peak of the $\mathrm{x}$-ray beam signal. From figure 5-7 it is clear that the signal due to scattered light is less than $0.5 \%$ of the peak signal at all six positions. As expected, the glare signal is relatively higher at the edges (positions a \& f) compared to the positions near the center of the crystal.

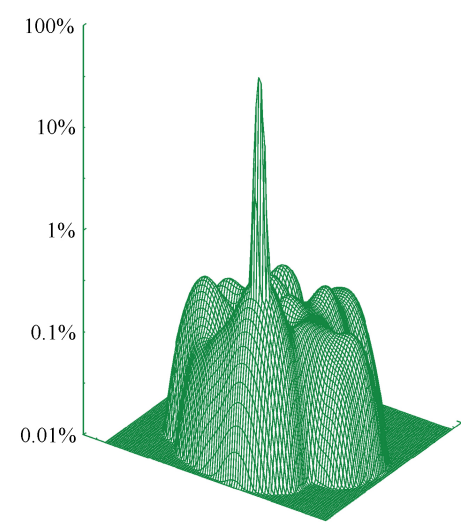

a)

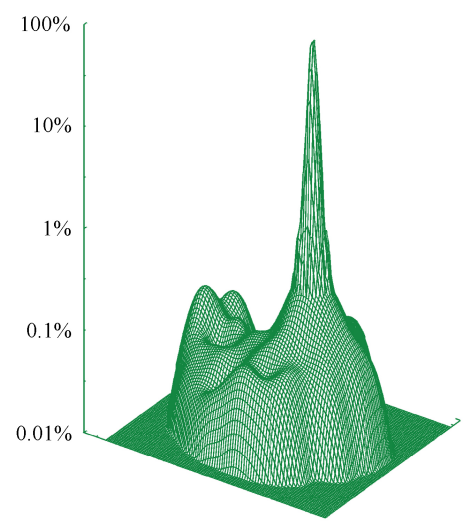

d)

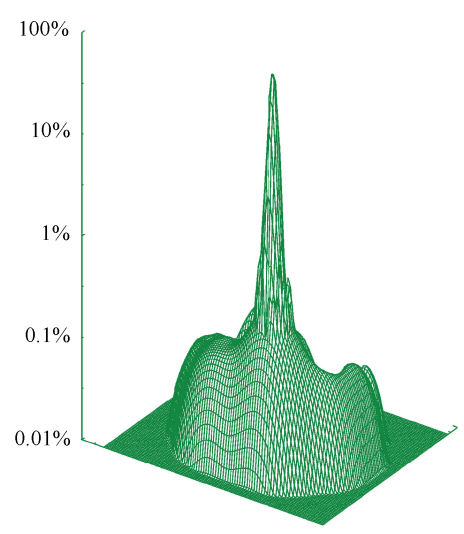

b)

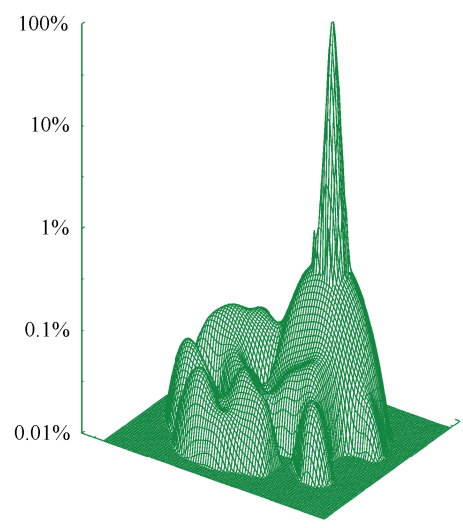

e)

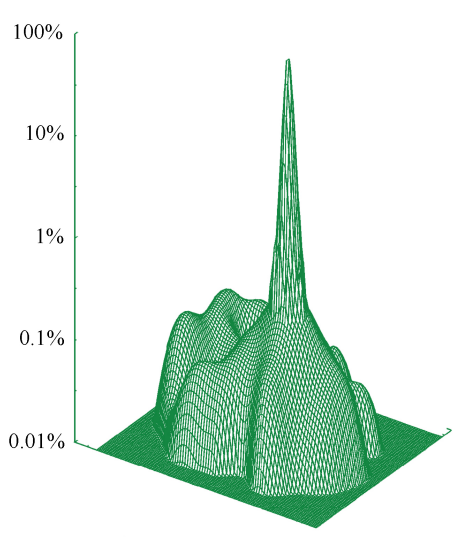

c)

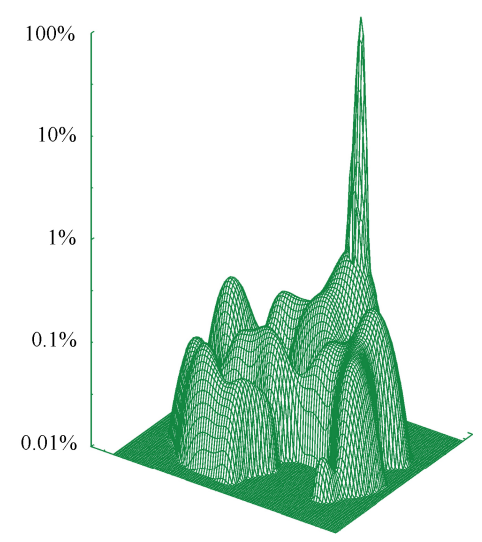

f)

Figure 5-7. Surface plots of light scatter glare for six positions of a highly collimated 6 MvP x-ray beam. Plots a) through $\mathrm{f}$ ) represent approximately equally spaced positions, 1 through 6 , along the diameter of the CsI (Tl) crystal. 


\subsection{Mirror Glare}

Mirror glare is a phenomenon that occurs in TV based EPIDs, causing loss of image quality. ${ }^{56}$ Mirror glare is caused due to light rays reflecting from the $45^{\circ}$ mirror back into the detector screen and then being detected by the TV camera, contributing a significant amount of false signal. ${ }^{57}$ The main reason why mirror glare is a significant source of false signal in TV based EPIDs is that these EPIDs use a powdered phosphor screen, which is white. Such a screen serves as an excellent reflector for the light rays coming from the mirror, thus generating significant levels of spurious signal. In contrast, the CsI (Tl) - CCD system is relatively insensitive to mirror glare because of the rough machined surfaces and the black coating in the back and side surfaces of the CsI (Tl) crystal. Figure 5-8 shows the paths taken by the light rays in case of a phosphor screen based system and the CsI (Tl) - CCD system.

The insensitivity of the CsI ( $\mathrm{Tl}$ ) system to mirror glare can also be seen from figure $4-2 b$ (chapter IV), which is a photograph of the CsI (Tl) crystal. Although a camera flash was used to obtain the photograph, the image of the $\mathrm{CsI}(\mathrm{Tl})$ crystal in the $45^{\circ}$ mirror appears completely black, which indicates that the black coated surfaces of the CsI (Tl) are highly effective in absorbing any stray light that reflects off the mirror. 


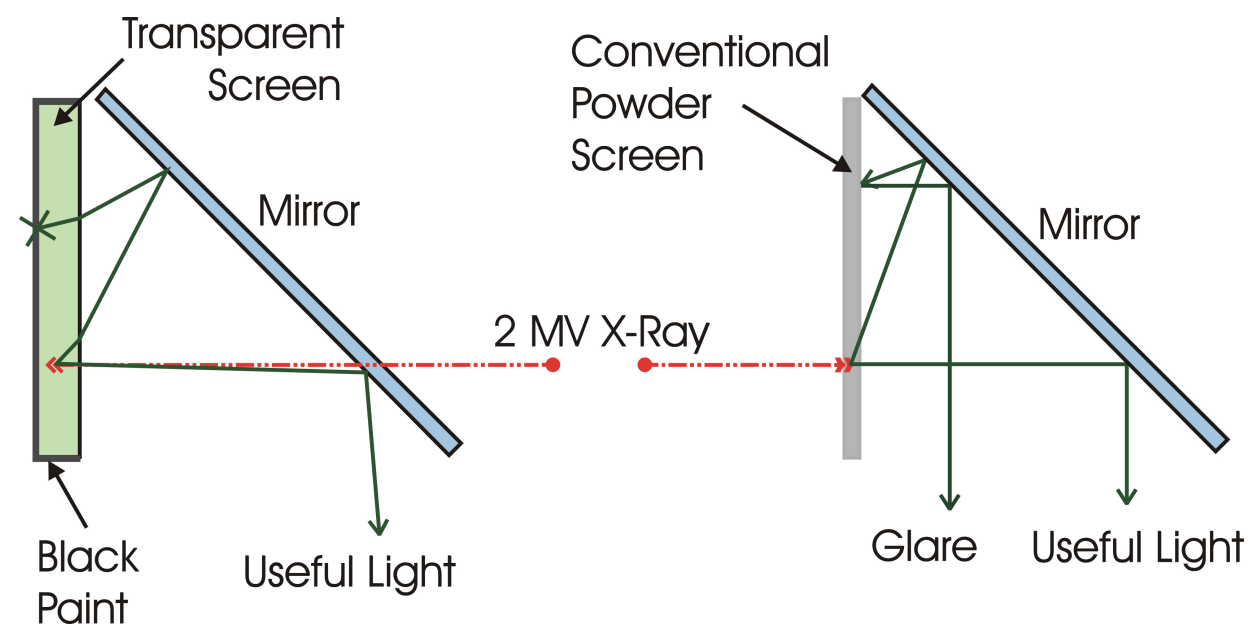

Figure 5-8. Mechanism of mirror glare in phosphor screen based EPIDs. In contrast, the $\mathrm{CsI}(\mathrm{Tl})$ screen is coated black and therefore absorbs the light that reflects back from the mirror

The relative intensity of the mirror glare in the CsI (Tl) - CCD system was measured by directing a laser beam at the CsI (Tl) screen three different angles and acquiring the images of the scatter pattern generated. Similar measurements were made using a Kodak Lanex Regular x-ray intensifying screen and the corresponding scatter pattern images were acquired. The CsI (Tl) scatter pattern was then scaled by the maximum signal obtained using the Lanex screen. The resultant surface plots ${ }^{42}$ are shown in figure 5-9. It can be seen that the signal from the CsI (Tl) crystal is less than $4 \%$ of the signal from the Lanex screen, which demonstrates the insensitivity of the CsI (Tl) - CCD system to mirror glare.

It should be noted that the proposed system will have a leaded glass plate coupled to the CsI (Tl) screen instead of the Lucite plate. It is possible that due to better optical coupling and with added anti-reflection coating, the proposed system may have even lower glare. 


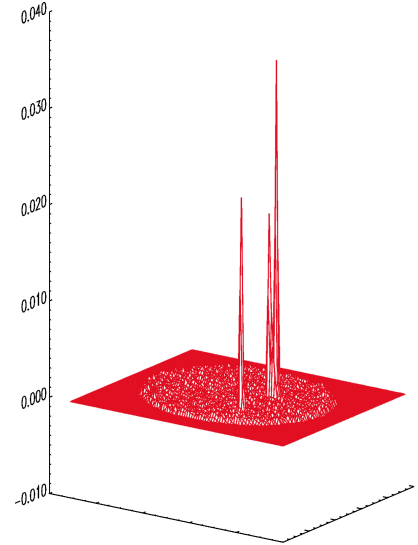

a)

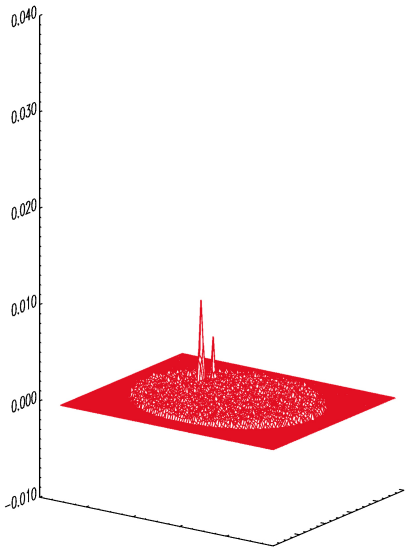

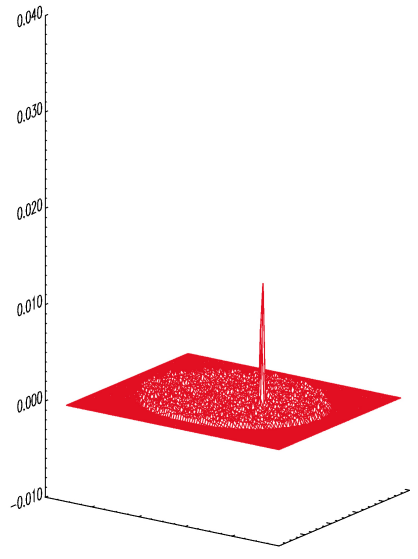

c)

Figure 5-9. Surface plots of mirror glare in the CsI (Tl) screen. The laser beam was pointed toward the CsI (Tl) screen at a) $30^{\circ}$ b) $45^{\circ}$ and c) $60^{\circ}$. The intensity values were scaled by the maximum intensity of the corresponding glare signal in the Lanex regular screen. 


\section{CHAPTER 6}

\section{Subject Imaging}

\subsection{Imaging Objects}

A qualitative evaluation of the image quality of images acquired using the CsI (Tl) - CCD portal imaging system was performed. The imaging objects consisted of an anthropomorphic phantom, a slaughtered pig and human subjects. Each object was imaged over a wide dose range in order to demonstrate the wide latitude of the CsI (Tl) - CCD system. Various linear and non-linear noise removal and image enhancement techniques (described in chapter VII) were used to process the images for optimal image visualization. The images acquired using the CsI (Tl) CCD system were compared with those obtained using Kodak ECL film and a commercially available EPID, Philips SRI-100.

\subsection{Chest Phantom}

Images of an anthropomorphic chest phantom were acquired at doses ranging from 1 monitor unit (MU) to $96 \mathrm{MU}^{41}$ Figures 6-1a and 6-1b show images of the chest phantom in the anterioposterior (AP) and the lateral view respectively. The AP images were taken at an x-ray magnification of 1.3 , while the lateral images were taken at magnification 1.4. both sets of images were acquired using a Nikkor $35 \mathrm{~mm}$, f 1.4 camera lens, giving a pixel size of $0.53 \mathrm{~mm}$ on the CsI (Tl) screen. From both figures it can be seen that the image quality remains fairly high over the entire dose range. Even at $1 \mathrm{MU}$ important anatomical details like ribs and other bony landmarks are clearly visible inspite of the 

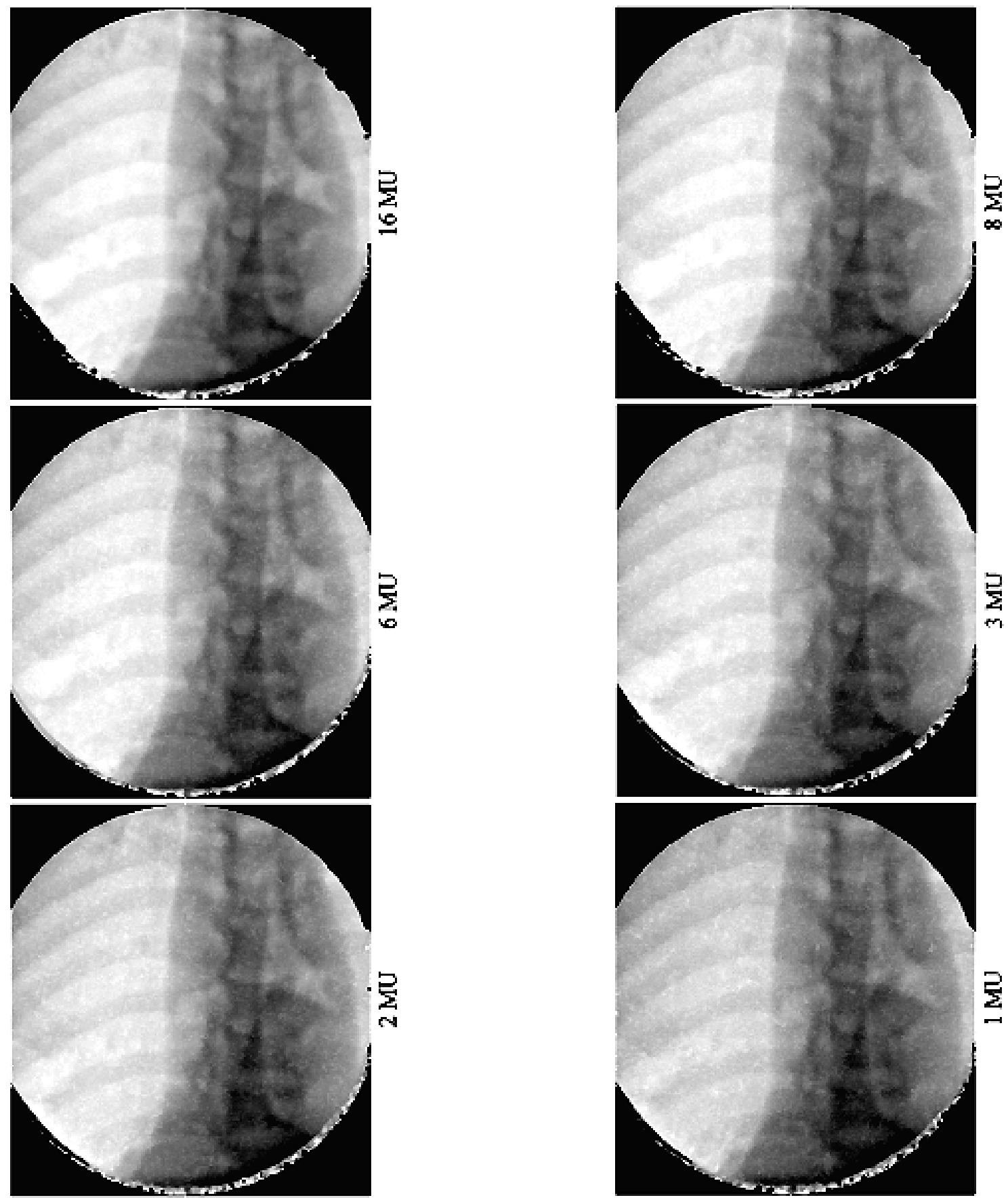

Figure 6-1. Images of a chest phantom. a) AP view of chest phantom. Image acquired using CsI (Tl) - CCD system using a Nikkor $35 \mathrm{~mm} \mathrm{f} 1.4$ lens. X-ray magnification $=1.3$. 

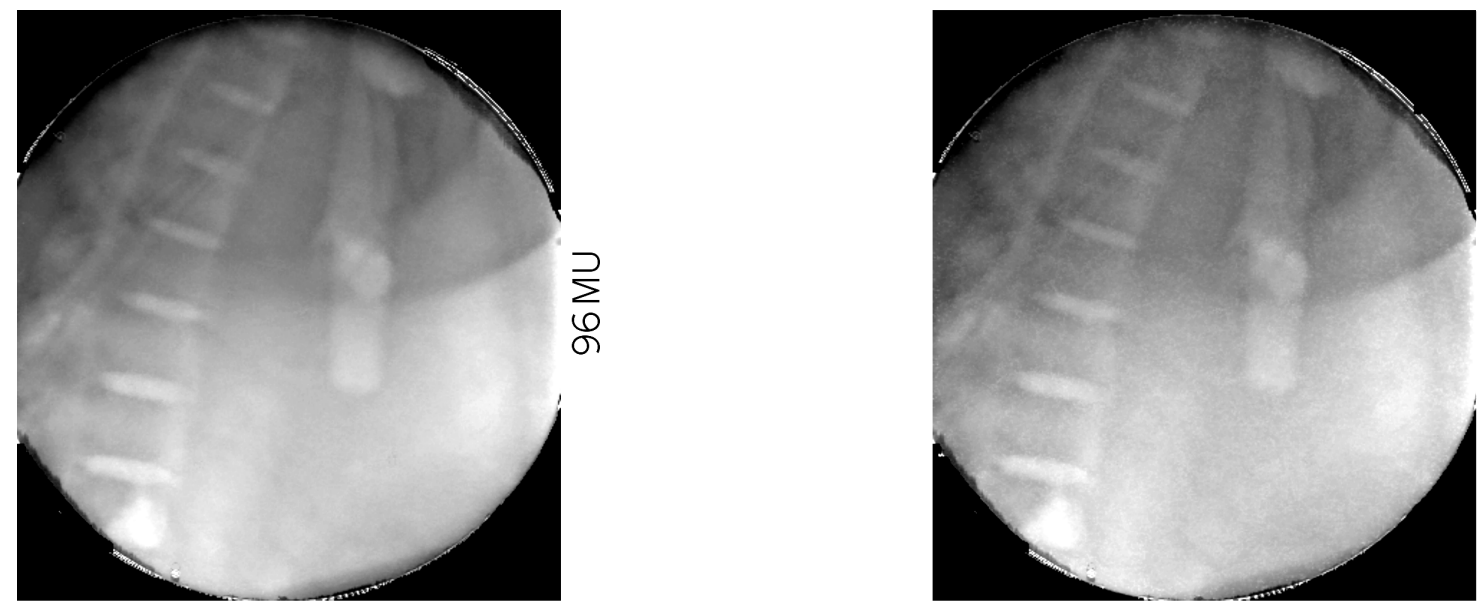

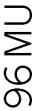

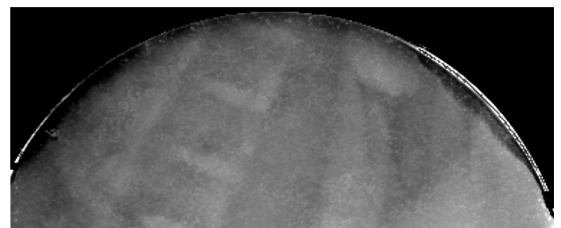

$\sum_{\infty}^{\supset}$

$\sum_{\forall}^{\supset}$
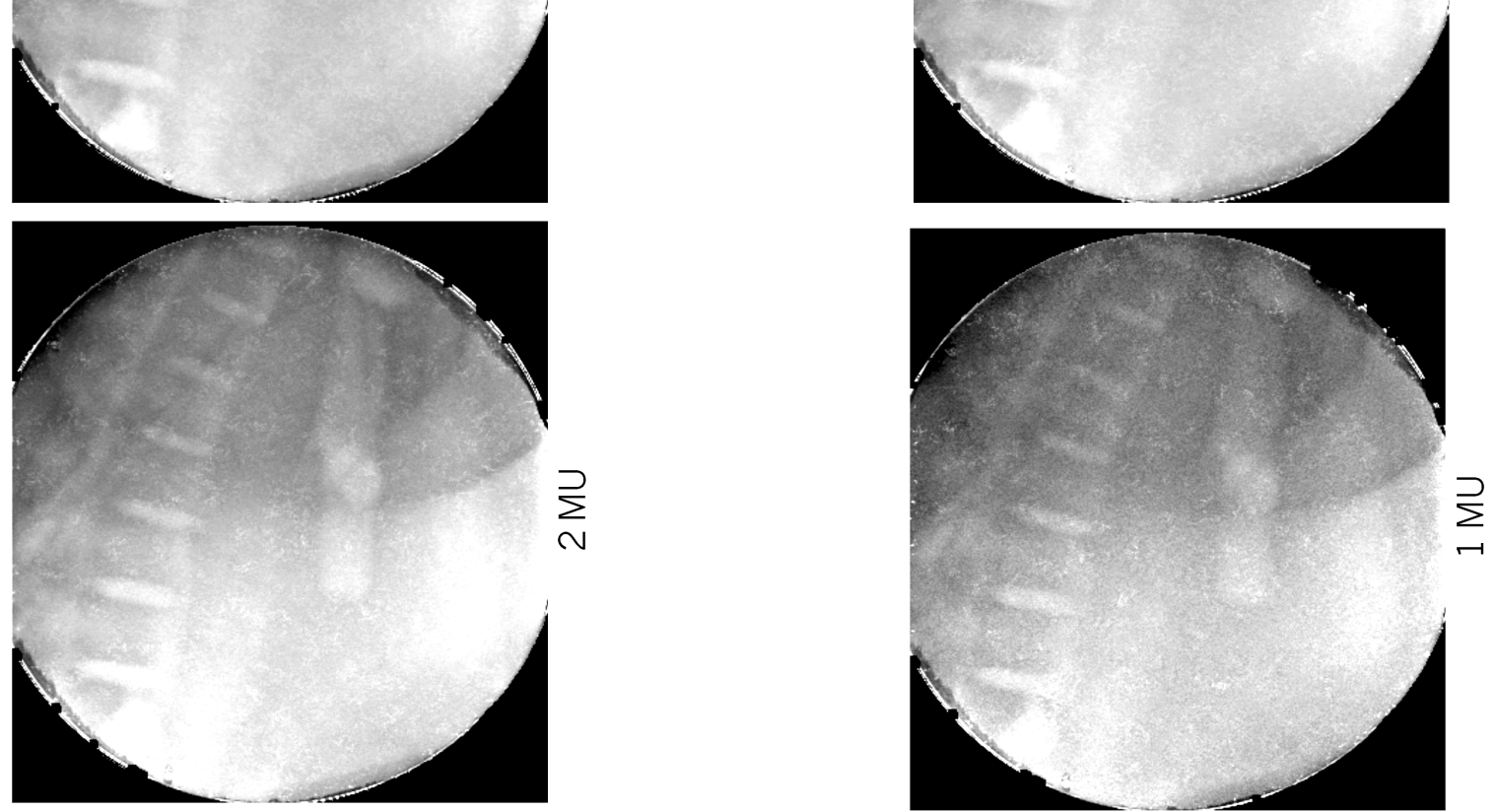

Figure 6-1:contd. b) Lateral view of chest phantom. Image acquired using CsI (Tl) - CCD system using a Nikkor $35 \mathrm{~mm}$ f 1.4 lens. X-ray magnification $=1.4$. 
relatively higher noise content compared to the images at higher doses. This ability of the CsI (Tl) - CCD system to acquire high quality images at very low doses has dual importance in that it significantly reduces patient dose during initial verification and also allows the system to acquire a dynamic sequence of high quality images during therapy.

Figure 6-2 shows a comparison of AP images of the chest phantom acquired using the $\mathrm{CsI}(\mathrm{Tl})$ CCD system, high contrast Kodak ECL portal film and the Philips SRI $-100 .{ }^{42}$ All the three images were acquired at comparable doses. The Kodak ECL film image was photographed off an x-ray light box using an Olympus D-600 digital camera. Both, the digitized film image and the Philips SRI - 100 image were processed in the same way as the CsI (Tl) - CCD image. From figure 6-2 it can be seen that although the CsI (Tl) - CCD system has lower spatial resolution compared to film due to its greater pixel size

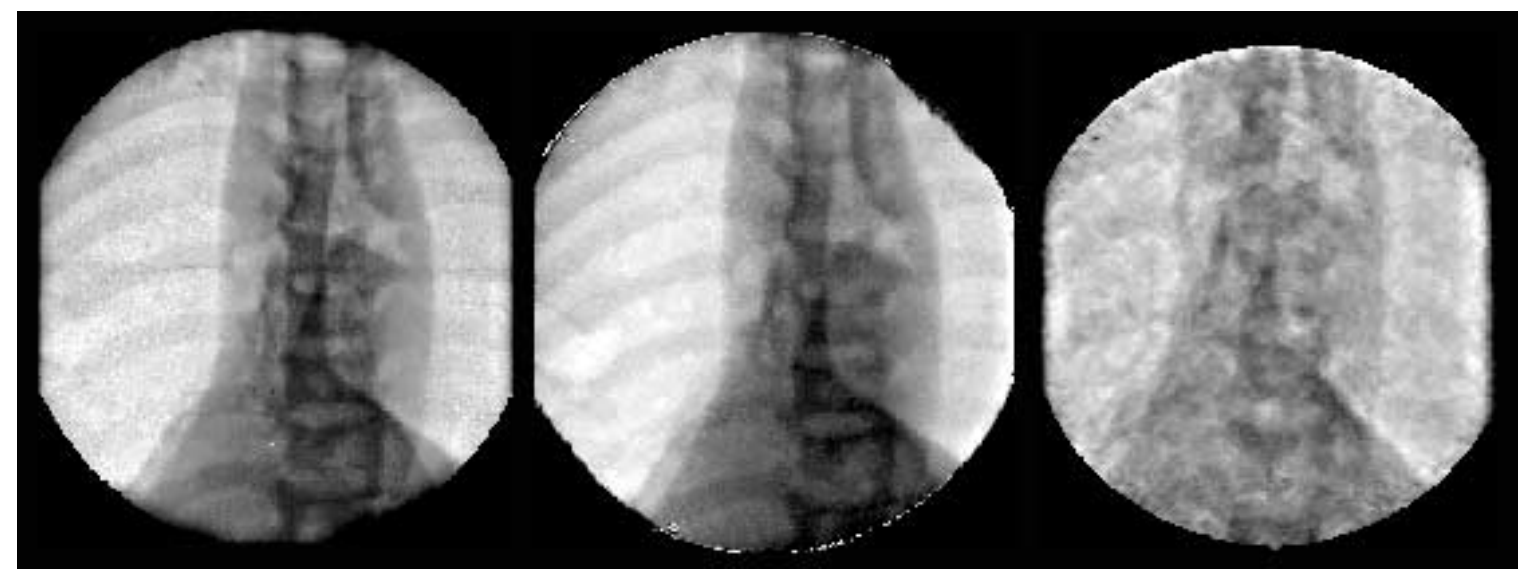

Kodak ECL Film: 20 MU

CCD-CsI (Tl): $16 \mathrm{MU}$

Philips SR-100 15 MU

Figure 6-2. Comparison of the CsI (Tl) - CCD system with ECL film and Philips SRI - 100 
$(0.53 \mathrm{~mm})$, the image quality of the CsI (Tl) system is comparable to ECL film and significantly superior to the Philips SRI - 100 EPID.

\subsection{Animal Imaging}

Figure 6-3 shows composite images of an entire leg of a pig. The images were acquired using the Nikkor 35 mm, f 1.4 camera lens, at an x-ray magnification of 1.4. Each composite image was made from a set of 6 images taken at consecutive positions from the apex of the foot to the acetabulum. Care was taken to ensure that there was a significant amount of overlapping region in consecutive images to avoid discontinuity in the composite image. The six image arrays (at each dose) were then appropriately concatenated to produce an image of the entire leg.

The signal intensities for each 6 image set lay over a very large range of values because the images near the apex of the foot were taken with a significant area of the field in air while in case of the images near the base of the leg, the primary beam passed almost entirely through bone and tissue. For adequate visual representation within the available grayscale range (0-255), a non-

linear function of the form $(\mathrm{A} \times \text { image })^{\mathrm{B}}$, where $\mathrm{A}=0.4$ and $\mathrm{B}=0.35$, was applied to the composite image.

From figure 6-3 it can be seen that fine anatomical details like the tibia, the knee joint and the bones in the foot are clearly visible even at the lowest dose (1MU), which demonstrates the high image quality that can be achieved by the CsI (Tl) - CCD system. 


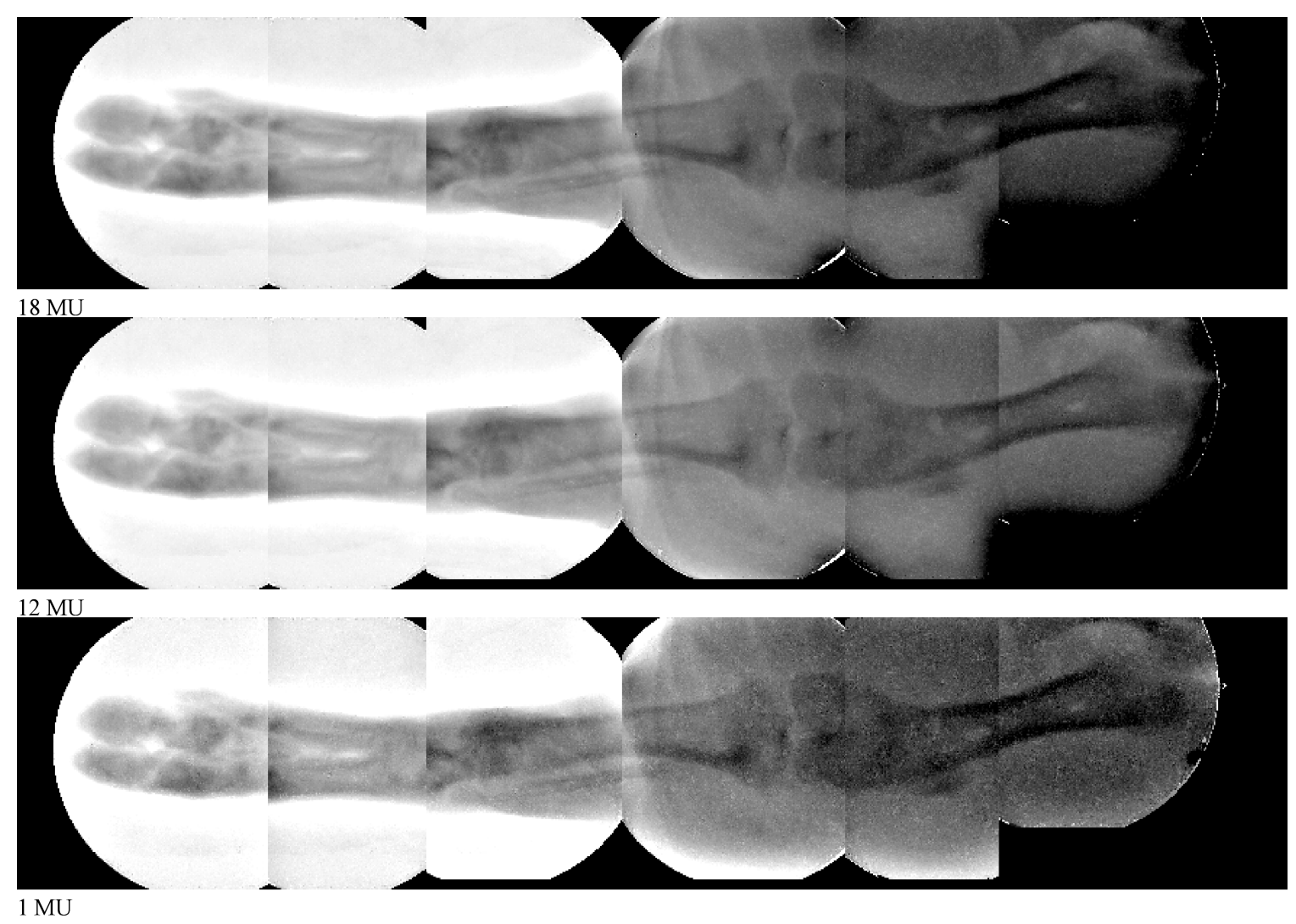

Figure 6-3. Composite image of the leg of a pig constructed from six views. The images were acquired using a Nikkor $35 \mathrm{~mm}$, f1.4 lens at an x-ray magnification of 1.4 .

\subsection{Human Subjects}

A limited clinical trial consisting of human subjects undergoing radiotherapy was conducted to evaluate the performance of the $\mathrm{CsI}(\mathrm{Tl})$ - CCD portal imaging system and perform a qualitative comparison of the prototype system with port film. ${ }^{42}$ The images were acquired using a high quality Angenieux $25 \mathrm{~mm}$ focal length, f 0.95, $16 \mathrm{~mm}$ movie camera lens, which gave a pixel size of $0.8 \mathrm{~mm}$ on the crystal, corresponding to an optical demagnification of 36. Although this larger pixel size should theoretically lower the spatial resolution, the images did not show any apparent loss of resolution. The Angenieux lens gave depth of focus of about $( \pm 2.5 \mathrm{~cm})$, which is in agreement with the theoretical value obtained using equation (2.4.2-4) in chapter IV. This 
large depth of focus would allow the use of any reasonable thickness of $\mathrm{CsI}(\mathrm{Tl})$ in a portal imaging system without loss of spatial resolution due to any portion of the CsI (Tl) screen being out of focus.

Figure 6-4 shows portal images for a lateral pelvic field. The subject was a 62 year old male being treated for prostate cancer with a conventional 4 field box technique utilizing $10 \mathrm{MV}$ photons from a Siemens MD2 linear accelerator and beam's eye view planning. Figure 6-4a shows the large field of view of the left lateral pelvic field acquired with a simulator using a 117 $\mathrm{kVp}$ diagnostic photon beam. This simulator image should display the highest contrast resolution due to the low photon energy. However, overexposure of the simulator film led to a significant loss in contrast resolution. In spite of this overexposure, the femoral head, acetabulum, sacrum and pubis symphisis are clearly visible, and serve as anatomical landmarks to align the patient in the treatment beam. Figure 6-4b displays the corresponding portal film image obtained using a $6 \mathrm{MV}$ photon beam and the customized cerrobend blocking to shape the reduced treatment field. Figure 6-4b has no image enhancement other than gray scale stretching. The white dots on figure $6-4 \mathrm{~b}$ are caused by the use of a graticule tray necessarily present in the port film mode of the linear accelerator. The graticule serves to provide a distance scale at the mechanical isocenter of the linac. The graticule is necessarily absent in the treatment mode, and hence not present in the CsI(Tl) images. The dramatic loss in contrast resolution in megavoltage imaging is apparent in Figure 6-4b. 


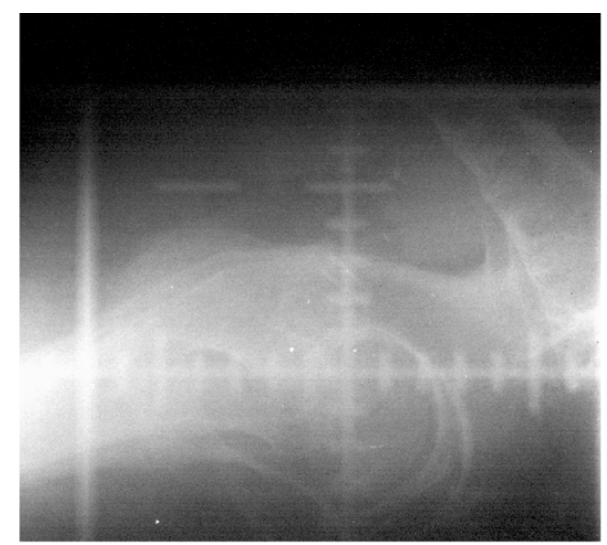

a) Diagnostic Film, $117 \mathrm{kVp}$

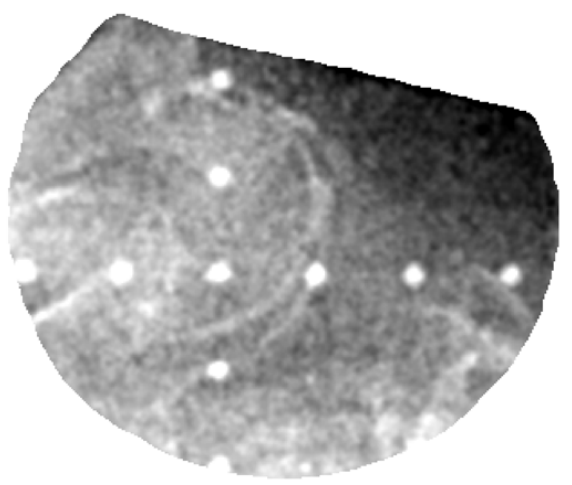

c) Portal Film (processed) $6 \mathrm{MV}, 10 \mathrm{MU}$

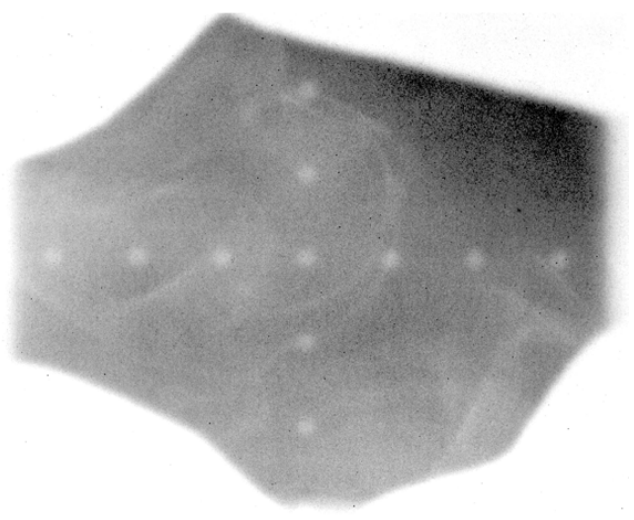

b) Portal film, $6 \mathrm{MV}, 10 \mathrm{MU}$ (unenhanced).

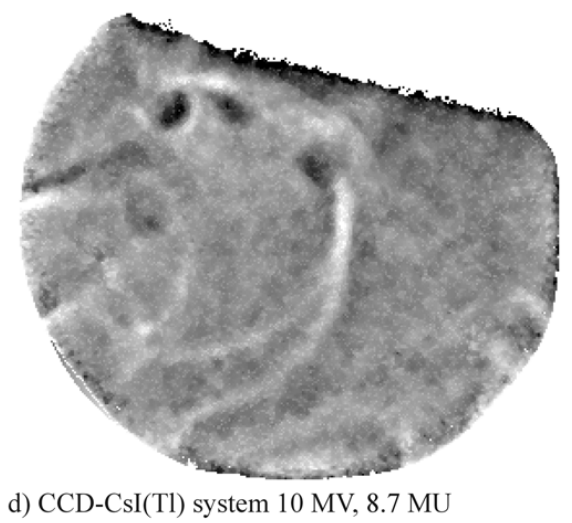

Figure 6-4. Lateral pelvic images: Comparison with portal film 
Figure 6-4d contains the $\mathrm{CsI}(\mathrm{Tl})$ based images. Of the useful anatomical landmarks, only the superimposed right/left femoral heads and acetabulums are in the field of view. Figure 6-4c contains the portal film equivalent image with the magnification, field of view, and image processing used in Figure 6-4d. The $\mathrm{CsI}(\mathrm{Tl})$ based image clearly shows superior contrast resolution compared to the portal film image, even though the portal film image was acquired using a $6 \mathrm{MV}$ photon beam while the $\mathrm{CsI}(\mathrm{Tl})$ image was acquired using a $10 \mathrm{MV}$ beam. The superimposed outlines of the left/right and femoral heads and acetabulums are clearly visible for the $\operatorname{CsI}(\mathrm{Tl})$ case.

Figure 6-5 contains $\mathrm{CsI}(\mathrm{Tl})$ based images taken at different monitor unit (MU) exposures. The indicated monitor units have been normalized to the case of $141 \mathrm{~cm}$ for the source to image distance, which is standard for our linac port films. The factor of 1.38 reduction in the CsI(Tl) images was calculated assuming that the plastic housing of the imaging system would be placed at the same position as the film cassette. The images in Figure 6-5 clearly show that the CsI(Tl) prototype is capable of acquiring clinically useful images at even $1 \mathrm{MU}$. Acquiring images at such a low dose would be important for dynamic portal imaging. 


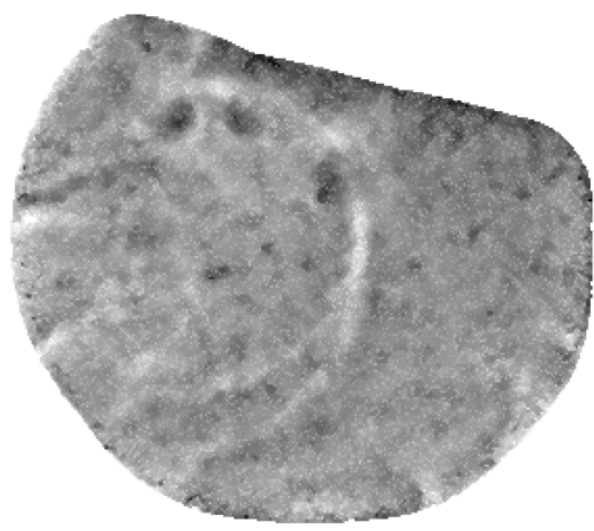

$0.72 \mathrm{MU}$

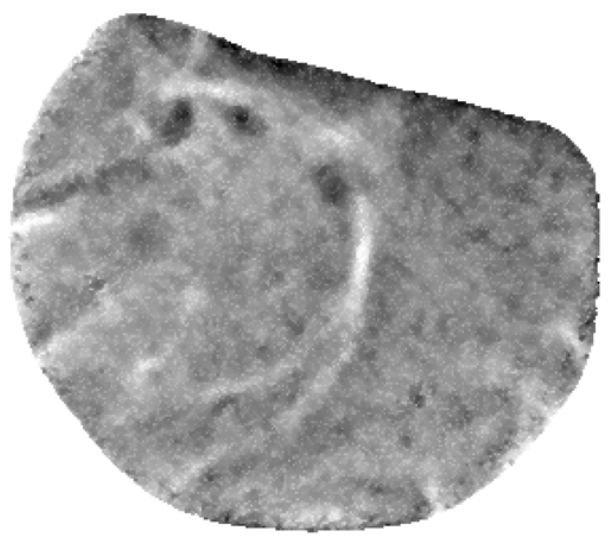

$2.9 \mathrm{MU}$

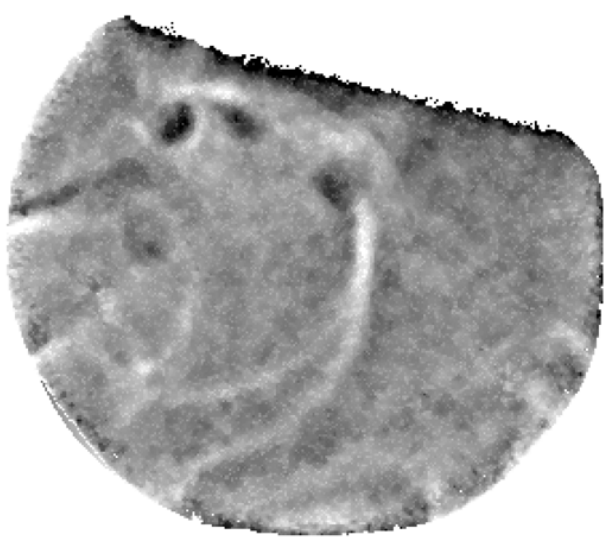

8.7 MU

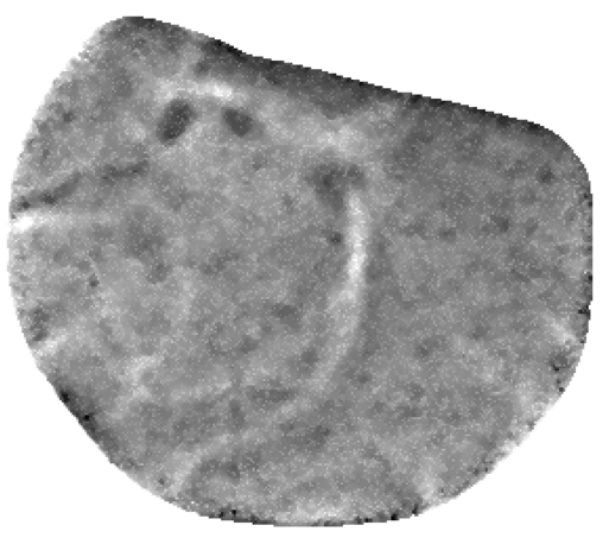

$1.5 \mathrm{MU}$

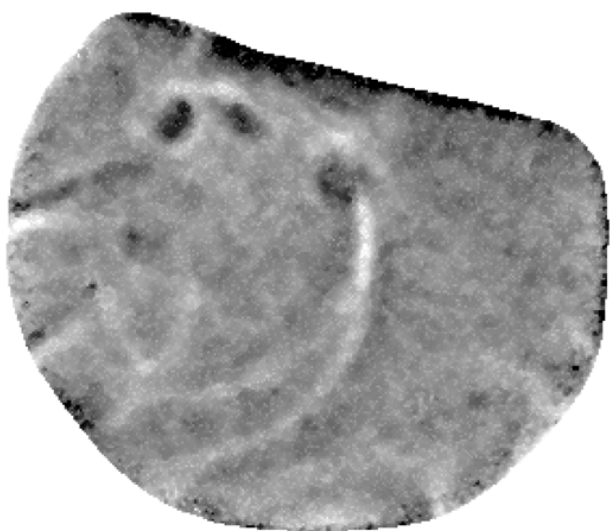

$5.8 \mathrm{MU}$

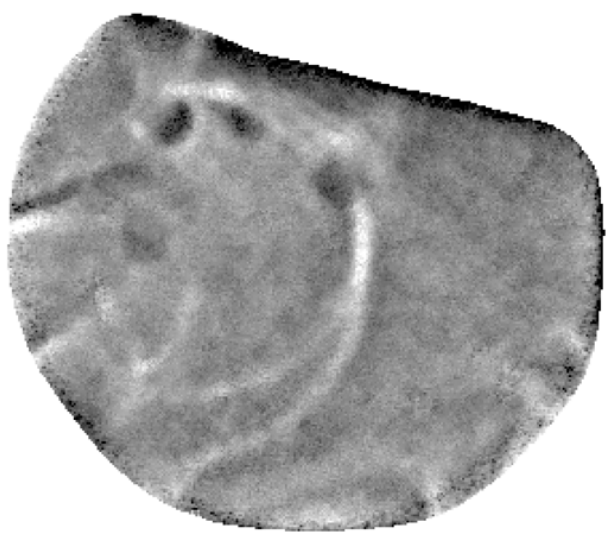

17.4 MU

Figure 6-5. Lateral images of the pelvic region at $10 \mathrm{MV}$, using the CCD-CsI(Tl) EPID. The patient was a 62 year old male treated for prostate cancer. Since the EPID had a greater source to detector distance (166 $\mathrm{cm})$ as compared to portal film $(141 \mathrm{~cm})$, the EPID doses were divided by a factor of 1.38 to obtain doses corresponding to the portal film distance of $141 \mathrm{~cm}$. 


\section{CHAPTER 7}

\section{Image Processing}

\subsection{Image Processing Protocol}

An image processing protocol was developed to process the images acquired using the prototype CsI (Tl) EPID for optimal image visualization. The protocol was developed after a careful study of the various types of noise (described in chapter IV, section 3) that degrade image quality in the CsI ( $\mathrm{Tl})$ portal imaging system. Image processing was performed in three main stages, viz. preprocessing, noise removal and image enhancement. Each stage was developed so that it could be either executed independently or seamlessly integrated with other stages for a completely automated image processing application. In the present work, the image processing was performed in a semi-interactive manner i.e. following the first approach of executing each stage separately.

\subsection{Pre-processing}

Stationary or fixed pattern noise is a common phenomenon in digital x-ray detectors. The sources of fixed pattern noise in the CsI (Tl) - CCD system have been described in chapter IV, section 3.1. Fixed pattern noise is removed using a technique called flat fielding.

In the CsI (Tl) - CCD system, flat fielding was implemented in the following manner. A set of background images (i.e. images taken without any object) was acquired. A dc offset signal corresponding to the dark current and the thermal noise was subtracted from each image. The offset signal was obtained from 16 columns at each side of the CCD 
array, which were not exposed to light (see chapter IV, section 2.4.3). The background images were averaged (to reduce random uncertainty) to give a single image $I_{b g r}(x, y)$, corresponding to the average background signal. The image of the object was acquired and the offset signal was subtracted from the image as described above, to yield the raw image, $I_{\text {raw }}(x, y)$. To obtain images at high doses, several low dose images were averaged to form $I_{\text {raw }}$. The flat fielded image was then obtained as

$$
I_{F F}(x, y)=\frac{I_{r a w}(x, y)}{I_{b g r}(x, y)}
$$

\subsection{Noise Removal (Impulse Noise)}

The CsI (Tl) - CCD system was affected by impulse noise which was caused by scattered high energy photons directly hitting the CCD camera. Although the effect of impulse noise was somewhat mitigated by using lead shielding (see chapter IV, section 3.2.3), a significant level of impulse noise (less than 8 - 10\%) was still present in the images. Typically, impulse noise is removed using median filtering. However, ordinary median filtering tends to blur fine details in an image. Since fine details may contain important clinical information, it is necessary that they be preserved during the filtering operation. Therefore, in this work an adaptive median filtering algorithm was developed. The algorithm took into account the variability of the entire image to ensure that the impulse noise was removed while fine structures were retained. The adaptive median filtering algorithm is explained in detail in chapter 8 . 


\subsection{Image Enhancement}

As explained in chapter I, megavoltage x-ray images inherently suffer from low contrast resolution and low spatial resolution. In order to improve the clinician's ability to determine placement errors, it is necessary to use digital contrast enhancement and edge enhancement techniques for optimal image visualization. In this work, linear contrast stretching, histogram equalization/specification and unsharp masking edge enhancement were used to improve image visualization.

\subsubsection{Linear Contrast Stretching}

Linear contrast stretching is a linear scaling of the gray values in the image such that they occupy the entire grayscale available for image display. Thus, for a $2 \mathrm{D}$ grayscale image $I(x, y)$, linear contrast stretching is implemented as

$$
I_{\text {strch }}(x, y)=\frac{I(x, y)-\min [I(x, y)]}{\max [I(x, y)]-\min [I(x, y)]} \times\left(\max _{\text {gray }}-\min _{\text {gray }}\right)
$$

where $\max _{\text {gray }}$ and $\min _{\text {gray }}$ represent the maximum and minimum values of the display gray scale.

\subsubsection{Histogram Processing}

For a digital image with gray levels [0, L-1], the histogram can be represented as a discrete function $\mathrm{p}\left(\mathrm{r}_{\mathrm{k}}\right)=\mathrm{n}_{\mathrm{k}} / \mathrm{n}$, where $\mathrm{r}_{\mathrm{k}}$ is the $k$ th gray level $(\mathrm{k}=0,1 \ldots \mathrm{L}-1)$ normalized between 0 and 1 , 
$\mathrm{n}_{\mathrm{k}}$ is the number of pixels at that gray level and $\mathrm{n}$ is the total number of pixels in the image. $\mathrm{p}\left(\mathrm{r}_{\mathrm{k}}\right)$ is the discrete form of the probability density function i.e. it gives an estimate of the probability of occurrence of gray level $r_{k}$. Thus the histogram is a plot of $p\left(r_{k}\right)$ for all values of $k$. Histogram equalization is based on the transformation of the form

$$
s_{k}=T\left(r_{k}\right)
$$

which produces a gray level $s_{k}$ for every level $r_{k}$ in the original image. Thus, histogram equalization attempts to create a uniform probability distribution by a non-linear one to one mapping of gray levels. For a discrete image, histogram equalization is implemented as

$$
s_{k}=T\left(r_{k}\right)=\sum_{i=0}^{k} \frac{n_{i}}{n}
$$

where $0 \leq r_{k} \leq 1$. An important property of equation 7.4.2-1 is that the transformation is independent of the input probability distribution ${ }^{58}$ i.e. $s_{k}$ will be the same irrespective of $r_{k}$.

Histogram equalization has a drawback that it gives only one result - an approximation of a uniform probability distribution. In some cases it is necessary to interactively specify the output histogram for optimal image enhancement. Such interactive enhancement is performed using a technique called histogram specification, which uses the inverse transformation of equation 7.4.2-1, denoted as

$$
r_{k}=T^{1}\left(s_{k}\right)
$$


If $\mathrm{p}\left(\mathrm{z}_{\mathrm{k}}\right)$ is the probability distribution of the desired image, the gray levels of the desired image could be equalized using the transformation

$$
v_{k}=G\left(z_{k}\right)
$$

and the inverse transformation would be given by

$$
z_{k}=G^{-1}\left(v_{k}\right)
$$

The inverse transformation in equation 3.2-4 would thus give the desired image. Since equations 7.4.2-1 and 7.4.2-3 both perform histogram equalization, both will yield the same probability distribution i.e. $\mathrm{s}_{\mathrm{k}}=\mathrm{v}_{\mathrm{k}}$. Therefore, equation 7.4.2-4 can be re-written as

$$
z_{k}=G^{-1}\left(s_{k}\right)
$$

Substituting the value of $s_{\mathrm{k}}$ from equation 7.4.2-1 in equation 7.4.2-5 we get

$$
z_{k}=G^{-1}\left[T\left(r_{k}\right)\right]
$$

From equation 7.4.2-6 it is clear that to obtain the desired image we need to determine the inverse transformation $\mathrm{G}^{-1}$. In practice, the inverse transformation is calculated using one of the following two approaches. In the first approach, the desired probability density function is specified and then digitized to form a histogram of the desired probability distribution. Using this desired histogram, a one to one mapping for the gray levels of the input image to the desired image is computed and stored. ${ }^{58}$ The second approach consists of interactively specifying the shape of the desired histogram using a graphic image processing software, which then computes the inverse transformation. 
This second approach was used to enhance the images acquired using the CsI (Tl) - CCD system. The CsI (Tl) images were imported into Adobe Photoshop (ver 5.0) and the output histogram was specified interactively using a mouse.

\subsubsection{Unsharp Masking Edge Enhancement}

The contrast enhancement techniques described in the previous two sections, by themselves, significantly improve image visualization. However, a substantial amount of edge enhancement is required along with contrast enhancement to significantly improve the clinician's ability to determine placement errors. ${ }^{59}$

In the present work, edge enhancement was implemented using a technique called unsharp masking. Unsharp masking is a high frequency boost technique used to emphasize sharp

transitions (edges). Unsharp masking was implemented by blurring the original image using a two dimensional gaussian kernel (which acted as a weighted average low pass filter) and taking the difference between the original image and the blurred image.

$$
I_{\text {diff }}(x, y)=I(x, y)-I_{\text {gauss }}(x, y)
$$

A multiple of this difference was added to the original image to give the final edge enhanced image.

$$
I_{\text {unsharp }}(x, y)=n \times I_{\text {diff }}(x, y)+I(x, y)
$$


The amount of edge enhancement is proportional to the magnitude of n. However, extremely large values of $\mathrm{n}$ tend to emphasize undesirable high frequency features like random noise, degrading the quality of the enhanced image. 


\section{CHAPTER 8}

\section{Adaptive Median Filter Algorithm*}

\subsection{Introduction}

Impulse noise causes individual pixels in an image to be corrupted. Typically, impulse noise is removed using a median filter because the median filter has a zero impulse response. Features such as edges and constant neighborhoods are invariant under median filtering, ${ }^{60}$ which makes the filter well suited for applications where suppression of impulse noise or heavy tailed noise is required but edges need to be preserved. The main drawback of the median filter is that it introduces a significant amount of signal distortion due to which fine details and thin lines are either lost completely or distorted. The loss of fine detail is highly undesirable in medical imaging because the details may contain important diagnostic information. A number of detail preserving algorithms based on the median filter have been developed such as weighted median filters, multistage max/median filters and multistage FIR median hybrid (FMH) filters. ${ }^{61,62}$ However, these methods tend to be somewhat complex in design and implementation. Also, in the above methods, a significant portion of the original data is altered. In most applications and especially in the area of medical imaging, it is important that after processing, the maximum amount of useful original data is retained and only the noisy data is discarded. The retention of a large percentage of the original data ensures high diagnostic value of the processed image. In this paper we discuss an adaptive median filter algorithm that preserves fine details in an image by

\footnotetext{
* Previously published in Proc. SPIE, Vol. 3661, Paper 132, entitled - Adaptive median filter algorithm to remove impulse noise from X-ray images and speckle from ultrasound images. Authors: A. Sawant, H. Zeman, D. Muratore, S. Samant. Reproduced with permission from SPIE.
} 
considering the local variability over the entire image and replaces less than 10 percent of the pixels in the original image.

\subsection{Development of the Adaptive Median Filter Algorithm}

\subsubsection{Median Filter}

The median filter, introduced by Tuckey, ${ }^{63}$ is a non-linear filter used in speech and image processing to remove impulsive noise while preserving edges. In image processing, two dimensional median filtering is used, which consists of running a $(2 \mathrm{~N}+1) \times(2 \mathrm{~N}+1)$ window $(\mathrm{N}=1,2,3 \ldots)$ over the entire image and replacing the central pixel at each position of the window with the median of all the pixel values that lie within the window. For an $(m \times n)$ image $f_{m, n}(x, y)$, we denote the median filtering operation using a $(2 \mathrm{~N}+1) \times(2 \mathrm{~N}+1)$ square window as

$$
f_{m, n}^{\prime}(x, y)=\operatorname{med}_{N}\left[f_{m, n}(x, y)\right]
$$

where $f_{m, n}^{\prime}(x, y)$ is the output of the filter.

A theoretical analysis of the median filter was presented by Gallagher and Wise ${ }^{60}$ where the authors defined the various input signals to a one dimensional median filter of window length $(2 \mathrm{~N}+1)$ as follows:

1. A constant neighborhood is at least $\mathrm{N}+1$ consecutive identically valued points such that the constant neighborhoods and edge together are monotone.

2. An edge is a monotonic region between two constant neighborhoods of different value. 
3. An impulse is a constant neighborhood followed by at least one, but no more than $\mathrm{N}$ points which are followed by another constant neighborhood. The two boundary points of these points comprising the impulse do not have the same value as the constant neighborhoods.

4. An oscillation is a sequence of points which is not part of a constant neighborhood, an edge, or an impulse.

Using these concepts Gallagher and Wise proved that the necessary and sufficient condition for any finite length signal to be invariant under median filtering was that the signal consist only of constant neighborhoods and edges. ${ }^{60,62}$ The above definitions and results can be easily extended to the two dimensional case. In case of an image an oscillation will be a fine structure or detail. Figure 8-1 shows the output of a median filter with different input signals. A median filter with a $(5 \times 5)$ window is applied at three different positions of the "image".
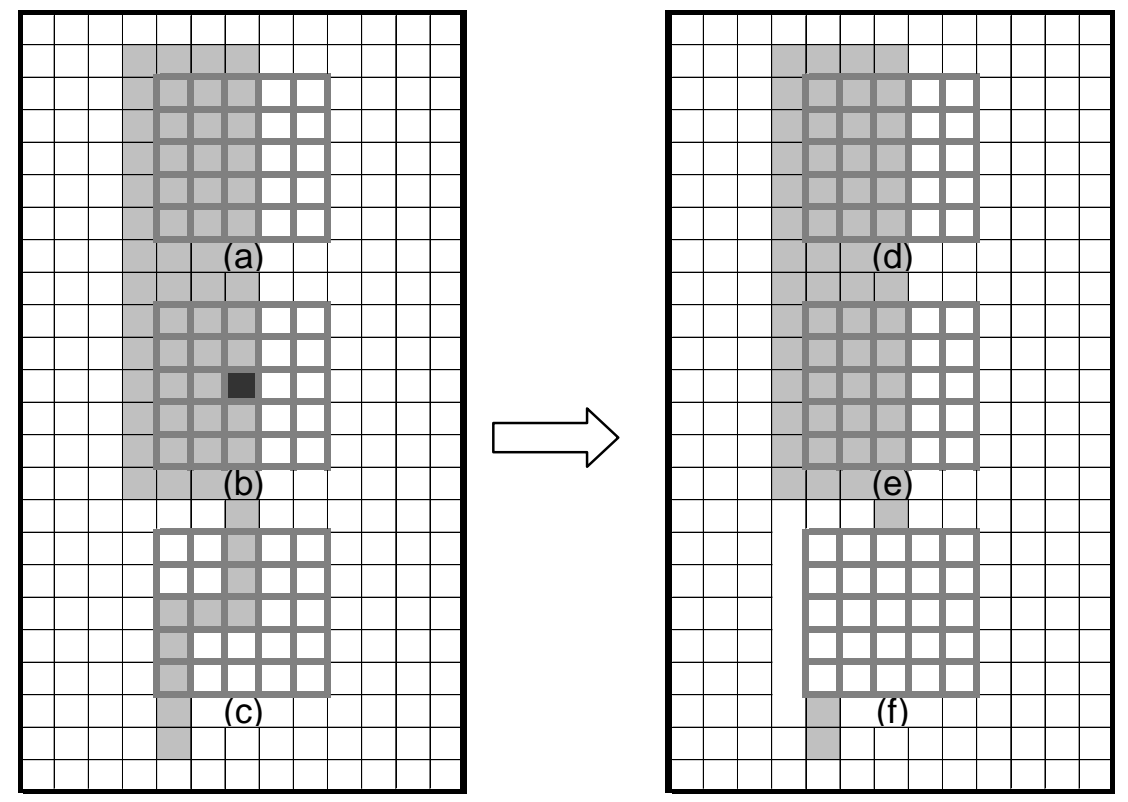

Figure 8-1. Outputs (d), (e), (f) of a $5 \times 5$ median filter with different input signals i.e. (a) constant neighborhood, (b) impulse and (c) fine structure. 
As expected the signal at (a) passes unchanged through the filter as shown in (d), since it consists purely of constant neighborhoods and an edge. At (b) the input signal is an impulse, which is replaced by the median value of the pixels within the window (e). Finally, at (c) the signal is a fine detail, which is lost completely after median filtering (f).

\subsubsection{The Thresholded Median Filter}

In the preceding section it was seen that median filtering distorts or removes fine details resulting in poor spatial resolution. A good algorithm to suppress impulse noise should be able to correctly identify the corrupted pixels and replace only those pixels with a reasonable value, leaving the useful original data unchanged, thus maintaining high spatial resolution. In a previous publication, ${ }^{64}$ a detail preserving algorithm called the 'choosing algorithm' was proposed. The algorithm aimed at suppressing impulse noise without significantly lowering the spatial resolution. The choosing algorithm was basically a thresholded median filter, where the absolute difference between the original image and its median filtered version at each pixel, was compared with a threshold value which was selected interactively. If the difference was greater than the threshold the pixel was replaced by the corresponding pixel in the filtered image, otherwise the original pixel was retained. The threshold was chosen such that only a small percentage (usually 10\%) of the pixels in the original image were replaced. The choosing algorithm thus preserved a large portion of the original data and was superior to the ordinary median filter in preserving detail. However, as shown in figure 8-2, this algorithm could not differentiate between high contrast fine structures and impulse or heavy tailed noise. In (a), the central pixel within the median filter window is part of a fine detail that has high contrast with respect to the background. In (b), the central pixel is an impulse that is comparatively lower in 
contrast. Therefore, the difference between the original image and the median filtered image will be greater in case of (a). As a result, if the threshold is chosen so as to remove the impulse, the fine structure will also be removed. On the other hand, if the threshold value is selected slightly higher, it is possible that the structure is completely lost while the impulse is retained. Also, automatic calculation of the threshold is impractical as explained in section 2.3.3.

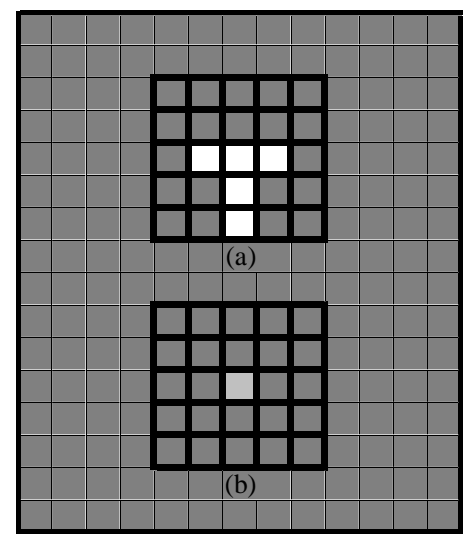

Figure 8-2. Worst case input for the choosing algorithm

\subsubsection{Adaptive Median Filter}

To overcome problems similar to those described in the previous section it is necessary for the filtering algorithm to be able to differentiate between fine details and impulse noise. The adaptive median filter described in this section, considers the local variability over the entire image and aims to replace only those pixels that are corrupted due to impulse noise, while preserving fine details. The algorithm is described below:

i) The image is median filtered and the absolute difference between the original image and the median filtered image is taken i.e. 


$$
d_{m, n}(x, y)=\mid f_{m, n}(x, y)-\operatorname{med}_{N}\left[f_{m, n}(x, y) \mid\right.
$$

ii) The original image is smoothed by convolving it with a two dimensional $(\mathrm{k} \times \mathrm{k})$ gaussian kernel, $g_{k, \sigma}(x, y)$.

$$
s_{m, n}(x, y)=f_{m, n}(x, y) * g_{k, \sigma}(x, y) \quad\{k<m, n\}
$$

The degree of smoothing is proportional to the value of $\sigma$.

iii) The absolute value of the difference between the original image and the smoothed image taken.

$$
v_{m, n}(x, y)=\left|f_{m, n}(x, y)-s_{m, n}(x, y)\right|
$$

This difference represents the variability over the image. A gaussian convolution is performed on $v_{m, n}(x, y)$ to obtain a smoothed version of the variability i.e.

$$
s v_{m, n}(x, y)=v_{m, n}(x, y) * g_{k, \sigma}(x, y) \quad\{k<m, n\}
$$

iv) The difference in (8.2.3-1) is divided by the smoothed variability to obtain a ratio

$$
r m, n(x, y)=\frac{d m, n(x, y)}{\operatorname{svm}, n(x, y)}
$$


This ratio at each pixel is compared with a threshold value. If the ratio is greater than the threshold then that pixel is replaced by its median filtered version, otherwise the original pixel is retained in the image. The threshold is automatically computed, such that only 8 to $10 \%$ of the pixels in the original image are replaced.

We can see that in (8.2.3-5), the numerator $d_{m, n}(x, y)$ will be high in two cases; when there is noise and when there is a fine detail. However the denominator $s v_{m, n}(x, y)$ will be small in case of noise (i.e. less overall variability) and relatively large in case of a fine detail (high variability). Hence this provides a kind of negative feedback which enables the filter to "see" fine details.

In the following subsections, we discuss some of the important design considerations in the implementation of the adaptive median filter algorithm.

\subsubsection{Filtering at the Edges}

A common problem in the implementation of median based filters is computing the filter response at the edges of the image. One approach in median based filters, is to append $\mathrm{N}$ pixels to each pixel on the edge before applying the filter. The appended pixels are equal in value to the pixel at the edge. ${ }^{60}$ However, this approach may not be very effective in case there is impulse noise on the edges. In such a case the appended pixels may be seen as false "structures". A more

robust approach is to reflect out the edges of the image ${ }^{64}$ i.e. assume the image to be periodic. For a filter width of $(2 \mathrm{~N}+1)$, at least $(\mathrm{N}+1)$ adjacent columns and rows including the edge are 
reflected. The reflected image is filtered and the central pixels that represent the original image are extracted. The reflection method ensures that any impulsive noise at the edges is filtered out.

\subsubsection{Gaussian Convolution}

The two dimensional Gaussian distribution (with zero mean) is given by

$$
g(x, y)=\frac{1}{\sigma \sqrt{2 \pi}} e^{-\frac{1}{2}\left(\frac{x+y}{\sigma}\right)^{2}}
$$

The gaussian distribution ranges from $-\infty$ to $+\infty$, hence it is necessary to truncate the kernel size within a reasonable value. This algorithm used a square gaussian kernel with each side $[2 \times 2(\sigma$ $+1)+1$ ] pixels wide, which means that the kernel was truncated at $\pm 2(\sigma+1)$. The choice of \pm $2(\sigma+1)$ as the cutoff point (rather than $\pm 2 \sigma$ ) ensured that a minimum integer valued width was assigned to the kernel in case $(\sigma<1)$. The edges of the image were reflected as described in section 2.2.1. Since convolution is a separable operation, in the actual implementation, gaussian convolution was performed in two steps. A one dimensional gaussian kernel of width $[2 \times 2(\sigma$ $+1)+1$ ] was convolved first along each row and then along each column. Using the property of separability reduced the time complexity from $\mathrm{O}\left[n^{2}\right]$ to $\mathrm{O}[n]$.

\subsubsection{Computing the Threshold}

In the choosing algorithm described in section 2.2., the threshold was chosen interactively by the user. In contrast, the adaptive algorithm calculates the threshold automatically such that 8 to $10 \%$ of the total pixels are replaced. This process is completely transparent to the user. Automatic threshold calculation is implemented in the following steps: 
i) A seed value is set for the threshold (typical value $=2$ ).

ii) The percentage of pixels that have $r_{m, n}(x, y)$ (see eq 2.3-5) above the threshold is computed. According to the algorithm, these pixels will be replaced.

iii) If percentage is greater than the upper limit (i.e. 10\%), the threshold is incremented and if the percentage is less than the lower limit (i.e. 8\%), the threshold is decremented.

iv) Steps (ii) and (iii) are repeated till the percentage of pixels to be replaced lies within the set limits.

To avoid an infinite loop it is important that the increment and decrement step sizes be unequal. For example if the threshold is incremented by 0.1 the step size for the decrement should be less than or equal to 0.05 . In all the images processed using this algorithm, the threshold was found to lie within a range of $[0.0-2.0]$. This small range allows small step sizes to be selected for increment and decrement of the threshold value, resulting in high accuracy with reasonable computation time.

In principle, automatic threshold calculation could also be used to calculate the threshold for the choosing algorithm. However in this case, since the threshold is compared with the absolute difference, the threshold varies over a large range of values for different images (typically over an order of magnitude). If the desired accuracy is to be maintained by keeping the increment and decrement step sizes small, the computation time could increase greatly. 


\subsubsection{Algorithm Parameters}

The adaptive median filter algorithm has two parameters, the width of the median filter window $(2 \mathrm{~N}+1)$ and $\sigma$ for gaussian convolution. The value of $\mathrm{N}$ determines the size of the neighborhood used to calculate the median value. The selection of $\mathrm{N}$ is similar to that in case of the ordinary median filter, e.g. for impulse noise that is $(2 \times 2)$ pixels wide $\mathrm{N}$ should be equal to 2 corresponding to a $(5 \times 5)$ window. The value of $\sigma$ determines the amount of smoothing performed by gaussian convolution, which in principle determines the fineness of detail preserved. It is difficult to compute the optimal value of sigma analytically, since one has to take into account the dimensions and shape of the finest structures, their contrast with respect to the background, the degree of non-uniformity of the background, etc. Thus the optimal value of $\sigma$ had to be determined empirically. After processing several images from the four different modalities discussed in this paper, it was found that the algorithm is fairly invariant with the value of $\sigma$. Obviously, the only condition is that the kernel size be less than the smallest dimension of the image, which puts an upper limit to the selection of $\sigma$. Therefore, $\sigma$ can lie within the range

$$
0<\sigma<\frac{1}{2}\left(\frac{n-1}{2}-2\right)
$$

where $n$ is the smallest dimension of the image. It should be noted that a larger value of $\sigma$ increases the computation time without any visible improvement in image quality. It is therefore advisable to select $\sigma$ within the range $(2 N+1) \pm N$. Since the output is not significantly affected by this parameter, $\sigma$ can be set to a fixed value. The ability to fix one parameter makes the adaptive median filter algorithm very robust. For all the images presented in this paper we have used $(\sigma=5)$. 


\subsubsection{Qualitative analysis using a test image}

A qualitative evaluation of the adaptive algorithm was performed by comparing it with a conventional median filter, as shown in figure 8-3. A (594 x 547), 8-bit grayscale image (a) was corrupted with impulse noise by randomly setting $5 \%$ of its pixels to white and 5\% to black. The impulse heights were chosen above and below the maximum and minimum values in the original image. The corrupted image (b) was filtered using a conventional median filter and the adaptive median filter ( $\mathrm{N}=2$ in both cases), to obtain the images in (c) and (d) respectively. The superior detail preserving ability of the adaptive algorithm can be seen by comparing the median filtered image and the adaptive filtered image. The former shows significant blurring while the latter is visually indistinguishable from the original image. Figure (e) shows the difference between the corrupted image and the median filtered image while (f) shows the difference between the corrupted image and the adaptive filtered image. Both (e) and (f) were windowed within 5\% of their grayscale range i.e.(2.5\% of the minimum and maximum). The difference image in (e) indicates significant loss of detail whereas no visible structure can be identified in (f), which demonstrates that the adaptive algorithm replaces only those pixels that correspond to noise.

A more objective measure of the detail preserving characteristics of the adaptive filter is seen in figure 8-4 which shows the histograms of the difference between the original image 8-3(a) and the filtered images. The histograms are plotted on a log-linear scale to accommodate the large range. Ideally, the filtered image should be identical to the original image. 


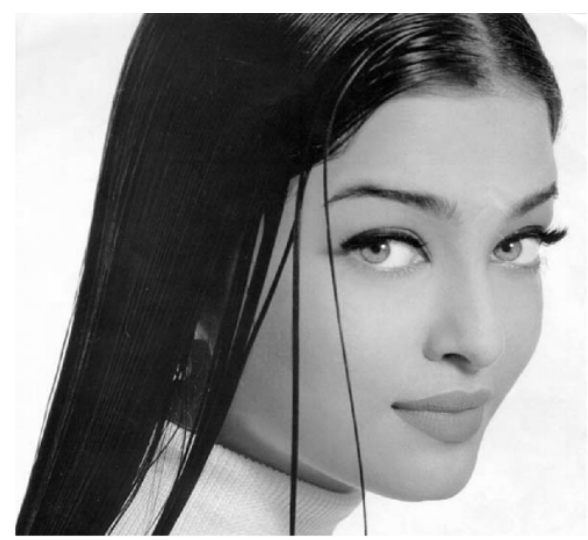

(a)

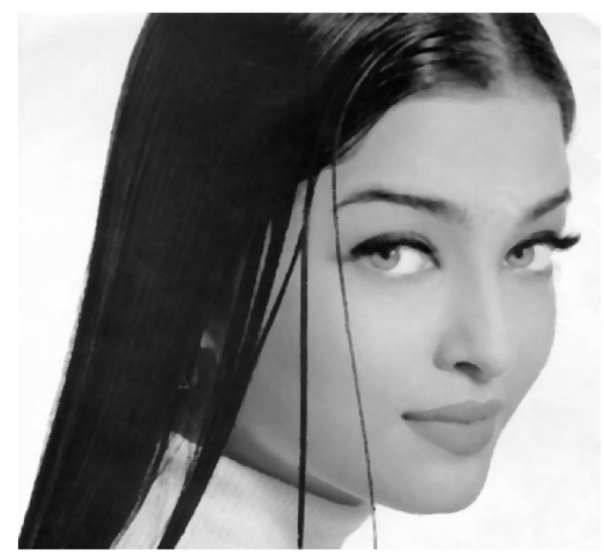

(c)

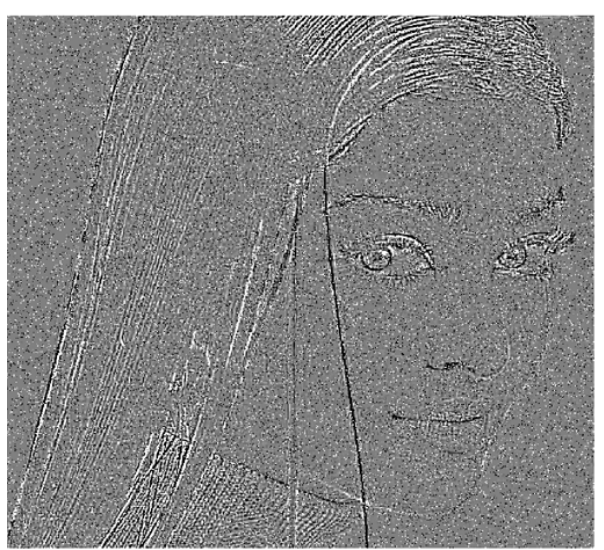

(e)

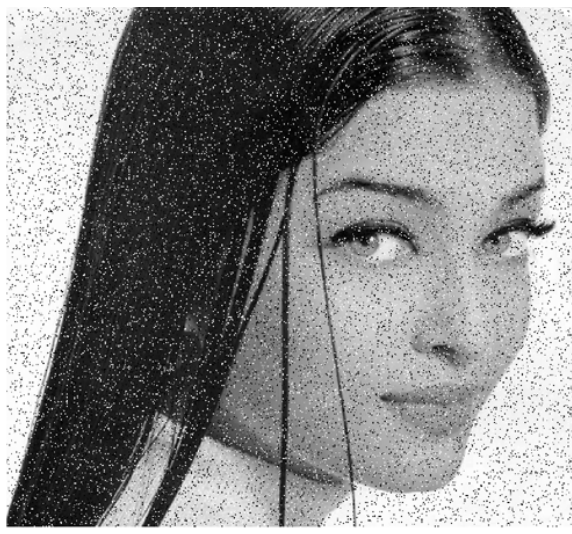

(b)

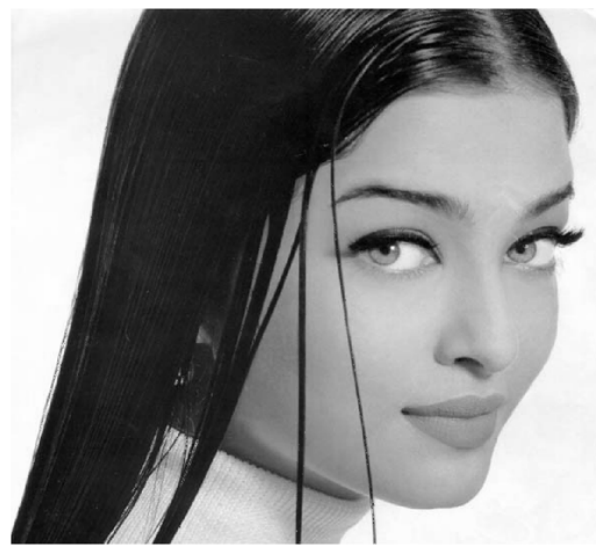

(d)

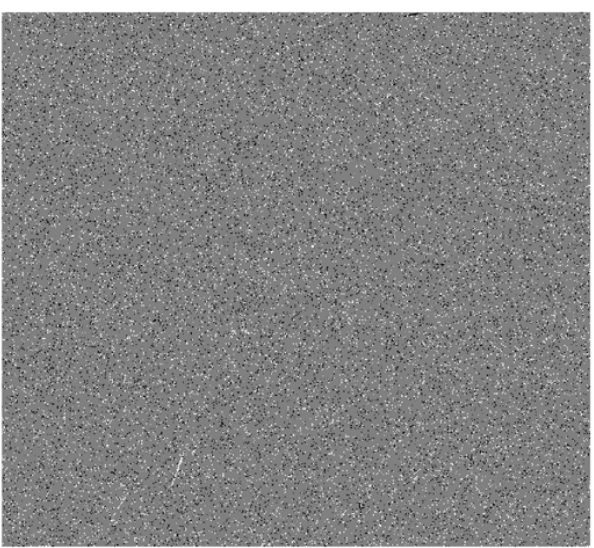

(f)

Figure 8-3 Comparison between the ordinary median filter and the adaptive median filter using an 8-bit grayscale test image. The original image is shown in (a). 10\% of the original image (a) was corrupted with impulse noise (b). Figures (c) and (d) show the filtered images using a median filter and the adaptive median filter respectively, with a 5 x 5 median filter window (i.e. $\mathrm{N}=2$ ) in both cases. Figure (e) shows the windowed difference image between the corrupted image (b) and the median filtered image (c). Figure (f) shows the windowed difference image between the corrupted image (b) and the adaptive filtered image (d). Both (e) and (f) were windowed within $2.5 \%$ of the maximum and minimum value of their respective differences. 


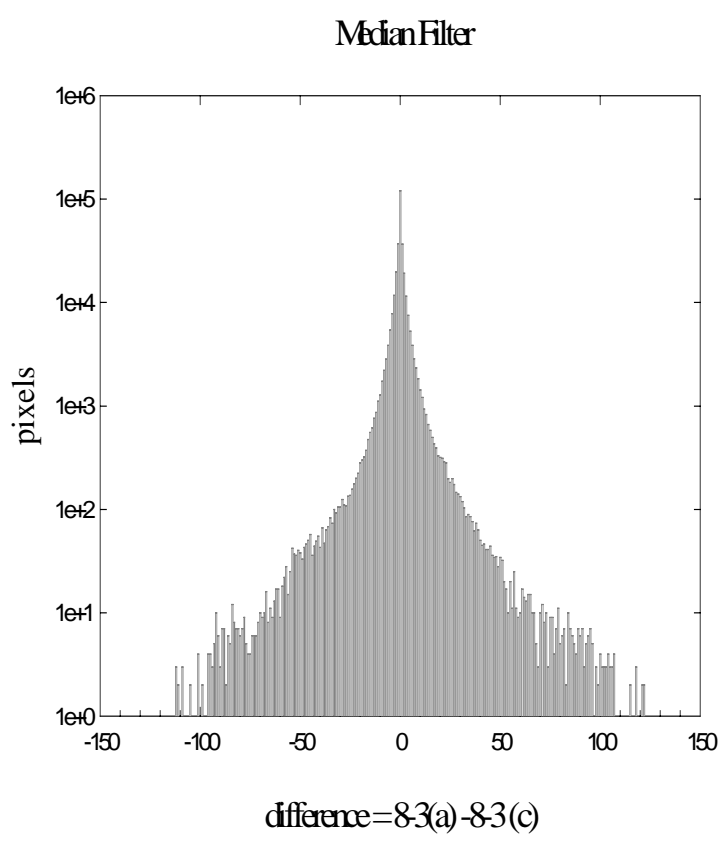

(a)

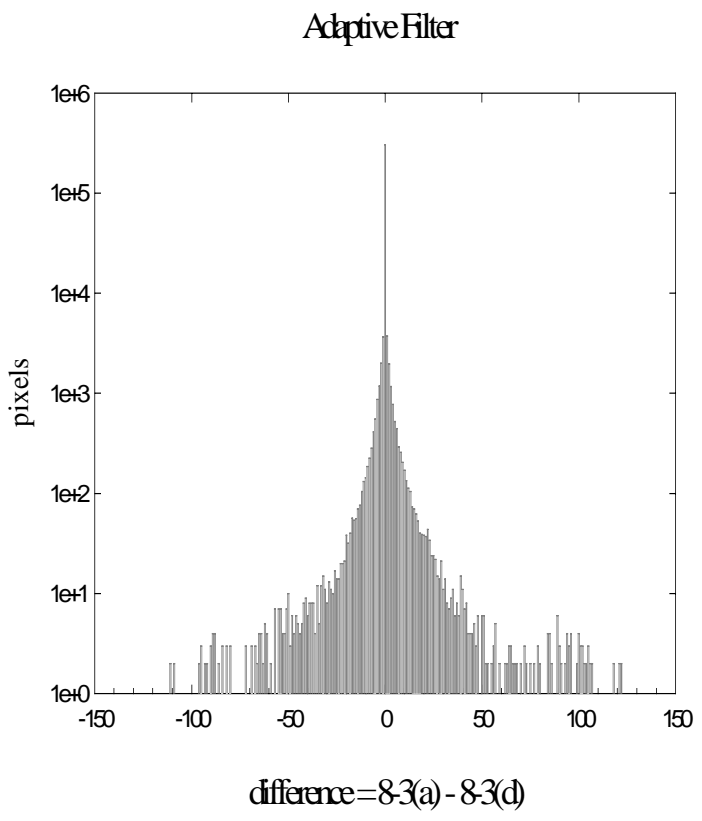

(b)

Figure 8-4. (a) Histogram of the difference between the uncorrupted image and the median filtered image. (b) Histogram of the difference between the uncorrupted image and the adaptive filtered image.

In this case the histogram will be a single peak at 0 . From figures 8-4(a) and 8-4(b) it is clear that the histogram corresponding to the adaptive filter resembles the ideal histogram more closely. The spread of values in 8-4(b) is significantly smaller than that in 8-4(a). Also, the ideal characteristic i.e. a single peak at 0 , begins more than an order of magnitude earlier in 8-4(a) as compared to 8-4(b), indicating that the adaptive algorithm approaches the ideal response more rapidly. 


\subsection{Processing Medical Images}

To demonstrate the robustness of the algorithm it was decided to try the adaptive filter on images from four different modalities, namely high resolution diagnostic x-ray images used for dual energy imaging, megavoltage x-ray images used for portal imaging, images obtained from an experimental high resolution CT scanner and ultrasound images used for image-guided spinal surgery.

\subsubsection{Dual Energy X-ray Imaging}

Images of a preserved foot of a rat, acquired by a high resolution digital radiography system ${ }^{65}$ were used in dual energy x-ray imaging for bone mineral densitometry. The imaging system consisted of a $\mathrm{Gd}_{2} \mathrm{O}_{3}(\mathrm{Eu})$ transparent ceramic scintillator coupled to a liquid nitrogen cooled CCD TV camera. Figures 8-5(a) and (b) show the unfiltered high and low energy images acquired using this system. The impulse noise is believed to be caused by radiation hitting the CCD. The high and low energy images were filtered using a median filter, a thresholded median filter and the adaptive median filter with $(\mathrm{N}=1)$ in resolution since in both cases only about $10 \%$ of the pixels are replaced. However, as explained in section 2.1, the thresholded median filter is unable to remove all of the impulse noise. This can be seen more clearly in the magnified view (f). The corresponding image (g) using the adaptive filter shows that the impulse is completely removed without lowering the spatial resolution. 


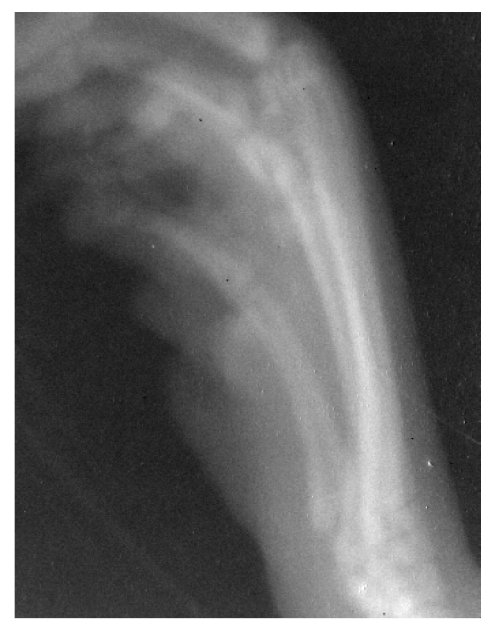

(a)

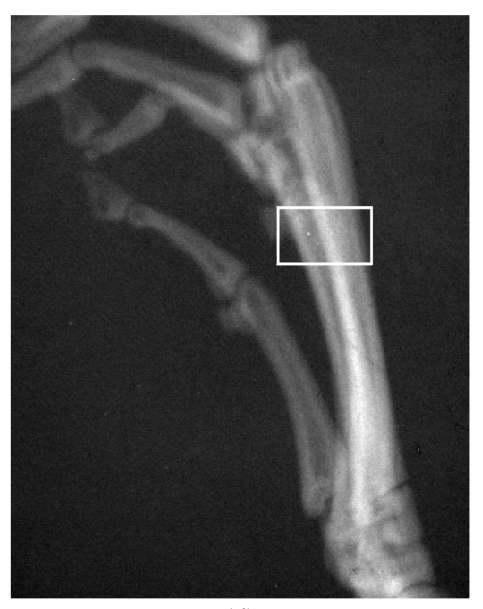

(d)

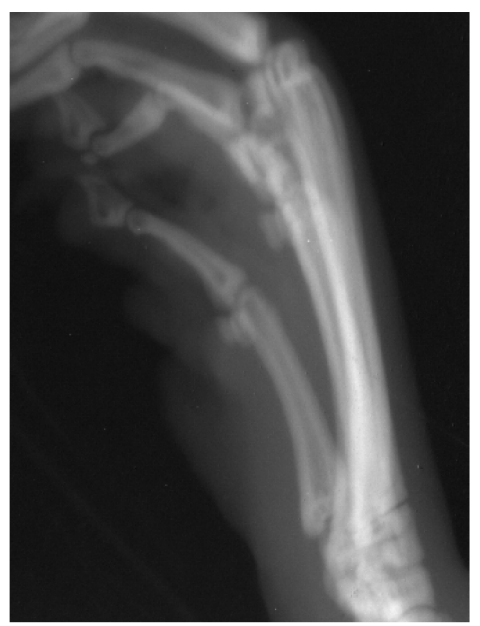

(b)

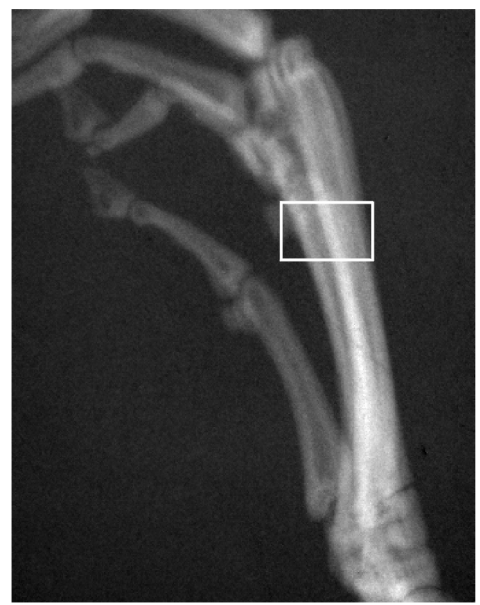

(e)

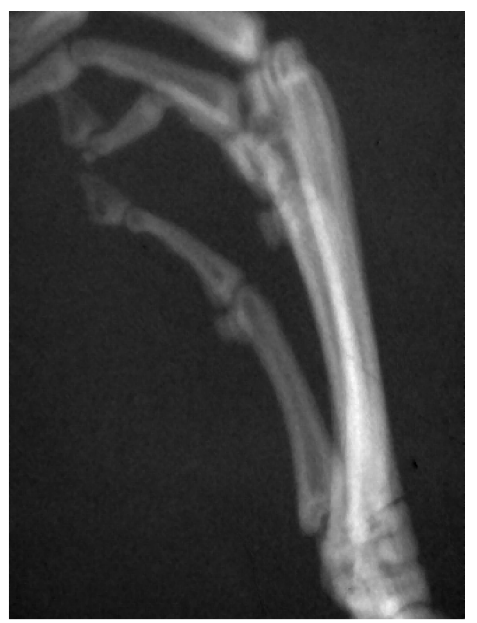

(c)

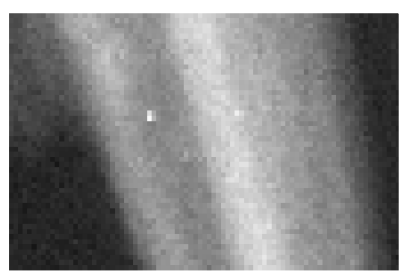

(f)

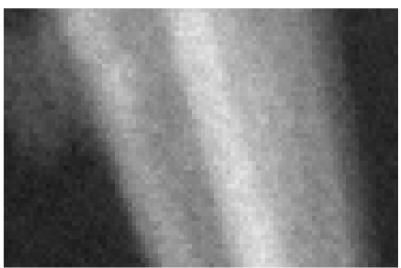

(g)

Figure 8-5. Dual energy X-ray images of a preserved rat's foot. (a) High energy image taken at $100 \mathrm{kVp}$, $15 \mathrm{~mA}, 48 \mathrm{sec}$, with $1.25 \mathrm{~mm} \mathrm{Cu}$ filtration. (b) Low energy image taken at $45 \mathrm{kVp}, 30 \mathrm{~mA}, 48 \mathrm{sec}$ with no added filtration. (c), (d) and (e) show the tissue subtracted images obtained by filtering the high and low energy images (a) and (b), using a median filter, thresholded median filter and the adaptive median filter respectively. The window size in all three cases was $3 \times 3$ (i.e. $N=1$ ). (f) and ( $g$ ) show the magnified views of the marked regions in images (d) and (e) respectively. The impulse is visible in (f) but cannot be seen in $(\mathrm{g})$. 


\subsubsection{Computed Tomography}

Figure 8-6 shows CT images of a slice of the head of an anesthetized hamster, acquired using an experimental ultra-high resolution CT scanner. ${ }^{66}$ The sinogram shown in (a) was used to obtain the reconstructed image (b) using back projection. An impulse in the sinogram caused a streak to appear in the reconstructed image. A magnified view (c) of the region in (a) within the box shows the impulse. To maintain the high resolution of the image, it is important that the filter used to suppress the impulse noise does not blur the fine details seen in the sinogram. These requirements are well satisfied by the adaptive filter. The filtered sinogram is shown in (d). The artifact in (b) is greatly suppressed in the reconstructed image (e), which uses the filtered sinogram. A magnified view (f) corresponding to (c) shows that the impulse is removed.

\subsubsection{Portal imaging}

Portal Imaging is used for the verification of localization of the treatment volume during radiotherapy. X-ray images taken in the therapeutic range (6 - $10 \mathrm{MV})$ suffer from poor contrast and spatial resolution (maximum $\approx 2$ line pairs $/ \mathrm{mm}$ ). Figure 8-7 shows an image of a pig's foot taken at $6 \mathrm{MV}$ and 18 monitor units $(1 \mathrm{MU} \approx 1$ centigray). The image was acquired with an experimental EPID (Electronic Portal Imaging Device) that used a $13 \mathrm{~mm}$ thick transparent CsI(Tl) crystal coupled to a liquid nitrogen cooled CCD TV camera. ${ }^{41}$ The unfiltered and filtered images are shown in (a) and (b) respectively. The impulse noise is caused due to stray radiation from the linear accelerator directly hitting the CCD. 


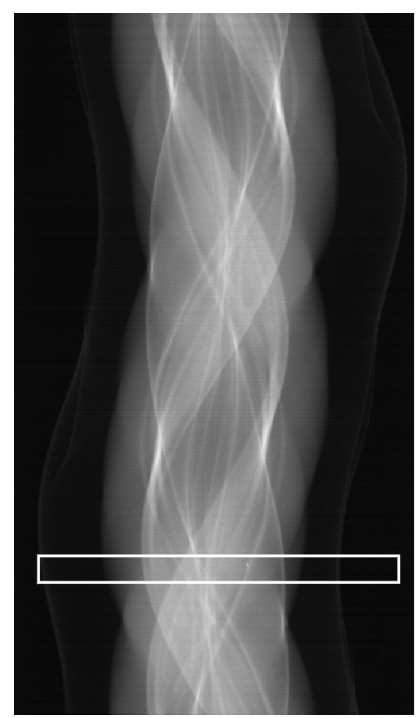

(a)

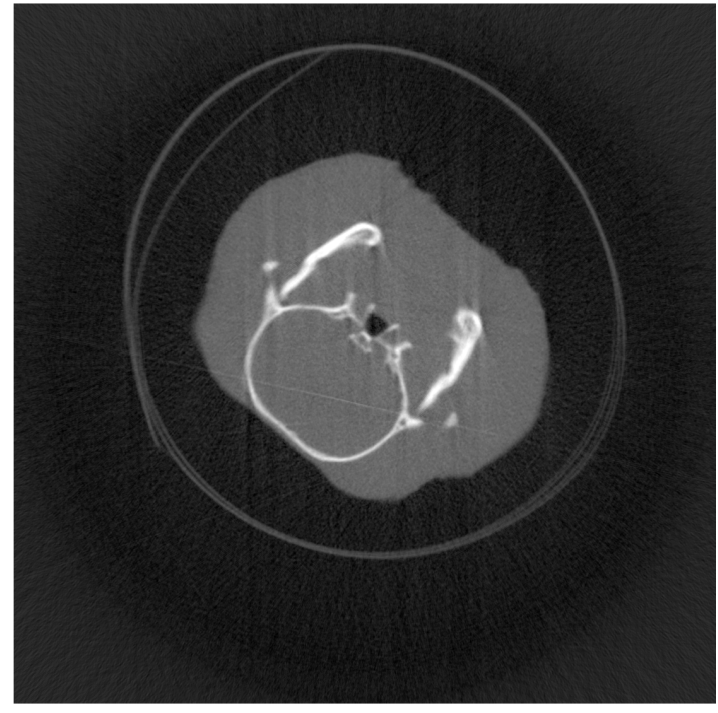

(b)

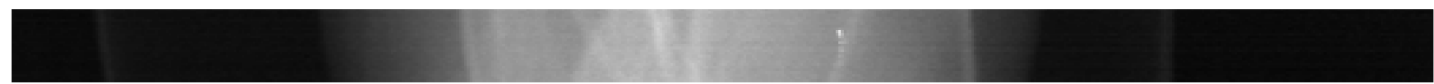

(c)

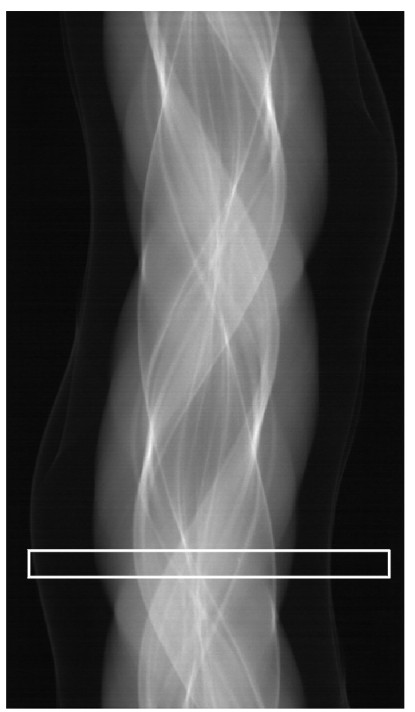

(d)

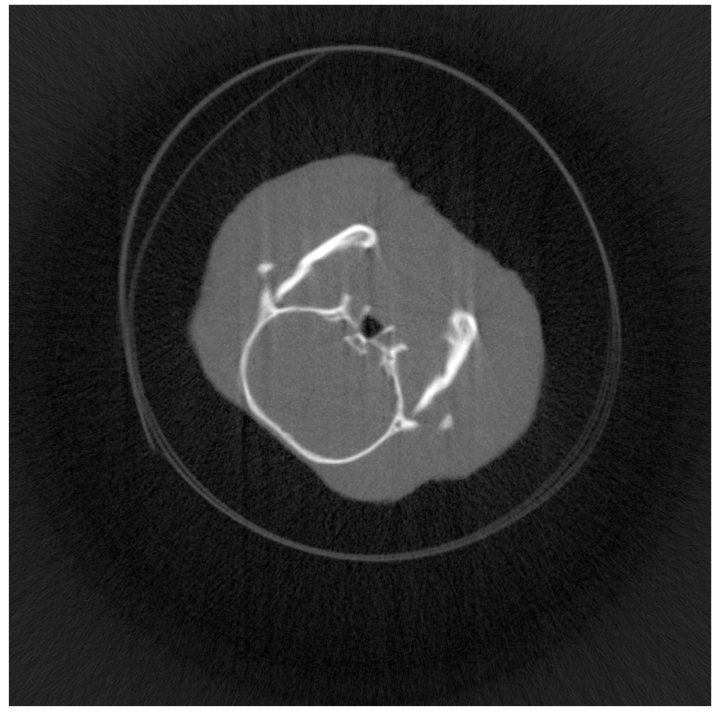

(g)

(f)

Figure 8-6. High resolution CT image of a slice of the head of a hamster. (a) Unprocessed sinogram taken at $65 \mathrm{kVp}, 200 \mathrm{~mA}$, with a scan time of 6 seconds. (b) Image reconstructed from (a). (c) Magnified view of the region in (a) within the box. The impulse in the projection image, seen more clearly in the magnified view, causes a streak to appear in (b). The sinogram was filtered using the adaptive median filter $(\mathrm{N}=2)$. The filtered sinogram (d) was used to obtain the reconstructed image (e). The artifact due to the impulse is significantly suppressed. The magnified view (f) of the region in (d) within the box, shows that the impulse is removed. 
Since portal images inherently have poor contrast resolution, a large amount of digital contrast enhancement and edge enhancement is applied to improve image visualization. Contrast enhancement was implemented using linear contrast stretching and histogram specification. Edge enhancement was implemented using unsharp masking. The original image was blurred using gaussian convolution (equation 8.2.3-2). The difference between the original image and the blurred image was taken, i.e.

$$
u_{m, n}(x, y)=f_{m, n}(x, y)-s_{m, n}(x, y)
$$

and a multiple of this difference $\left(3.5 \times u_{m, n}(x, y)\right)$ was added to the original image. Figures (c) and (d) show the contrast enhanced images corresponding to (a) and (b). Since unsharp masking acts as a high frequency boost filter, the impulse noise is seen much more pronounced in (c) as compared to (a). The filtered image does not show impulse noise even after contrast enhancement.

\subsubsection{Ultrasound}

An interesting application of the adaptive median filter was the suppression of speckle from vertebral ultrasound (US) images used for image-guided surgery. Surfaces were to be extracted from these images for a surface-based registration of intra-operative US images to pre-operative CT images. Ultrasound images suffer from multiplicative speckle noise. For low resolution images, speckle can be modeled as

$$
z(x, y)=f(x, y) \times s(x, y)
$$


where $\mathrm{s}(\mathrm{x}, \mathrm{y})$ is the speckle noise intensity. ${ }^{67}$ Speckle is caused by the destructive and constructive interference of returning echoes and the transmitted acoustic signal. The presence of speckle reduces the image quality by lowering the SNR and decreasing the image contrast, which makes feature recognition and extraction difficult and unreliable. Speckle reduction techniques include spatial averaging, homomorphic Weiner filtering and extreme sharpening combined with median filtering. ${ }^{67-69}$ In figure 8-8 we compare the speckle suppression properties of the adaptive median filter $(\mathrm{N}=1)$ with those of a $(3 \times 3)$ plus shaped morphological open operator. ${ }^{70}$ The latter is basically a shape operator which acts as a non-linear low pass filter. ${ }^{67,70}$ In the original image (a), which shows the side of the spinous process of the L2 lumbar vertebra, a significant amount speckle can be seen. For accurate and reliable surface extraction it is necessary that the speckle noise be removed while the fine structures, especially at the edges, are preserved. Figures (b) and (c) show the filtered images using the adaptive median filter and the open operator respectively. The image in (c) appears significantly more blurred than (b).

Surface extraction from (b) and (c) was performed by linear thresholding of the filtered images and edge detection was performed using ray tracing. ${ }^{70}$ Figures (d) and (e) show the surfaces extracted from the adaptive filtered and the open filtered images respectively. From the images it is clear that the adaptive filter is slightly better at speckle suppression (while preserving details). The surface information obtained using the adaptive filter is comparable to that obtained using the morphological open operator, which again demonstrates the robustness of the adaptive filter. 


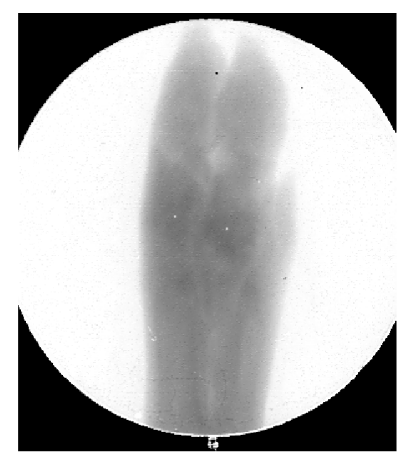

(a)

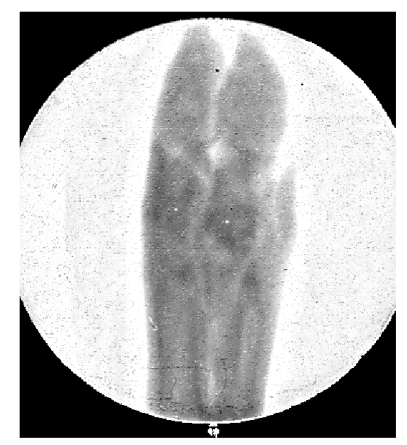

(c)

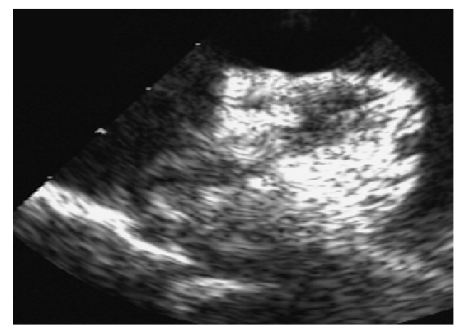

(a)

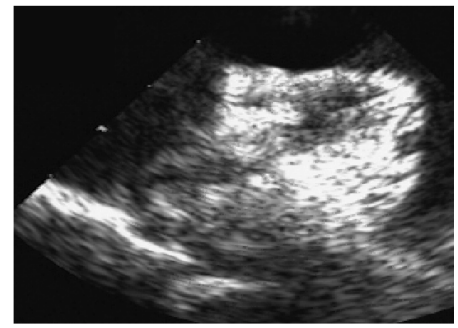

(b)

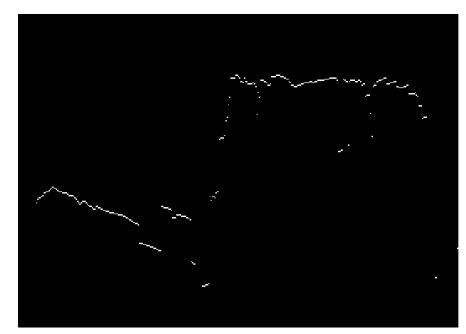

(d)
Figure 8-8. Ultrasound images taken with a 3.5 MHz phased array transducer. (a) Unprocessed image showing the side of the spinous process of the L2 lumbar vertebra. (b) Image filtered using the adaptive median filter $(\mathrm{N}=1)$. (c) Image filtered using a $(3 \times 3)$ morphological open operator. (d) and (e) show the surfaces extracted from the filtered images (b) and (c) respectively.
Figure 8-7. Portal images of a pig's foot taken at $6 \mathrm{MV}, 18 \mathrm{MU}$. (a) shows the unfiltered image which contains impulse noise. The adaptive filtered image $(\mathrm{N}=4)$ is shown in (b). Both the images are contrast enhanced using unsharp masking. The contrast enhanced versions of (a) and (b) are shown in (c) and (d) respectively.

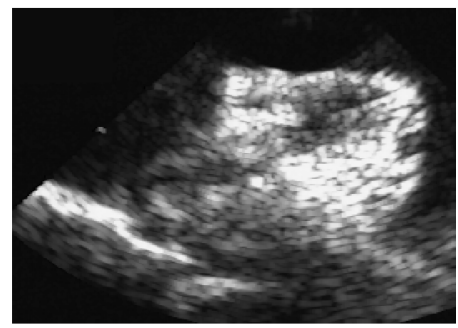

(c)

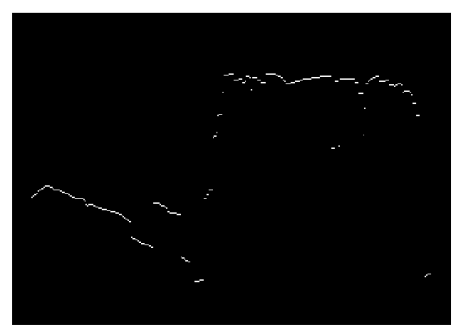

(e) 


\subsection{Conclusion}

In this work we described an adaptive median filter based algorithm that preserves fine details and retains more than $90 \%$ of the original information. A qualitative analysis showed that the adaptive filter is significantly superior to the median filter at preserving fine detail, thus maintaining high resolution in the output image. Of the two parameters, i.e. $\mathrm{N}$ and $\sigma$, it was shown that the latter could be assigned a fixed value. The adaptive median filter with a fixed value of $\sigma(\sigma=5)$, was used to process images from four different modalities. The ability of the adaptive filter to effectively suppress additive (impulse) as well as multiplicative (speckle) noise among many imaging modalities, with variation in only one parameter (i.e. $\mathrm{N}$ ) demonstrated the robustness of the algorithm. 


\section{CHAPTER 9}

\section{Future Work}

\subsection{Optimal Thickness of CsI (TI) and Leaded Glass for Proposed System}

From the theoretical calculations described in chapter III using the one dimensional Monte Carlo model, it was determined that the DQE and the QDE increase with screen thickness. However, increased screen thickness is accompanied by a corresponding loss in the spatial resolution. The upper limit of the thickness of the screen is determined by two factors viz. the depth of field and the spread of the light photon shower inside the crystal. In chapter IV it was shown that an adequate depth of field (up to $\pm 2.5 \mathrm{~cm}$ ) could be achieved using a lens with a relatively short focal length and a small $f$ number. The thickness of the screen will therefore be limited by the spread of the light photon shower inside the crystal. A detailed three dimensional model of the energy deposition profile for CsI ( $\mathrm{Tl}$ ) crystals of different thicknesses is required to get an indication of the level of spread of light photons with screen thickness, which in turn will facilitate the determination of an optimal screen thickness for the proposed portal imaging system. A validation of the optimal thickness obtained using the 3-D modeling can be performed experimentally by obtaining small CsI (Tl) crystals $(5 \mathrm{~cm} \times 5 \mathrm{~cm})$ and imaging a resolution bar pattern to determine the variation in MTF with detector thickness.

In the present work, the response of the detector has been considered uniform with respect to position from the central axis. While this assumption is a good approximation for the present detector $(20.32 \mathrm{~cm}$ in diameter), it is possible that a full field of view detector $(40 \mathrm{~cm}$ in diameter) may exhibit variation in spatial response away from the central axis. Three 
dimensional Monte Carlo modeling will be used to obtain an indication of the variation in spatial reponse with respect to distance from central axis for the full field of view CsI (Tl) screen.

Simulations will also be performed to determine the optimal thickness of leaded glass required in order to absorb the brehmstrahlung generated in the patient. The patient will be modeled as different thicknesses of water and a profile of the energy deposition in the CsI (Tl) screen due to secondary radiation (i.e. generated in the patient) will be obtained.

\subsection{Shielding}

The CCD in the present prototype system is affected by radiation noise due to scattered radiation from the head of the linear accelerator. As discussed in chapter IV, adequate shielding has been shown to significantly reduce the effect of noise due to such scattered radiation. ${ }^{44}$ It is required to perform Monte Carlo simulations using head scatter data from linear accelerators, to determine the optimal thickness of shielding to reduce the scattered radiation dose to an appropriately low fraction.

\subsection{Development of the Proposed Full Field of View EPID}

A portal imaging system with a $40.6 \mathrm{~cm}$ diameter transparent $\mathrm{CsI}(\mathrm{Tl})$ scintillator coupled to a $2048 \times 2048$ pixel, thinned, back surface illuminated frame transfer CCD camera from PixelVision will be designed and built using adequate shielding and a CsI (Tl) screen of optimal thickness. 
The proposed system will have an order of magnitude higher light collection efficiency compared to the Astromed CCD based system. This higher light collection efficiency will be due to two factors - higher light detection efficiency ( 0.9 compared to 0.3 for the Astromed) due to thinning of the CCD and less demagnification due to the greater surface area of the PixelVision CCD chip. The lower demagnification will allow the use of a lens with longer focal length. Therefore, for a given $\mathrm{f}$ number, a lens with a longer focal length will have an entrance pupil of a larger diameter (since $f$ number is the ratio of the focal length to the diameter of the entrance pupil). Thus, if a $50 \mathrm{~mm}$ lens is used in place of a $25 \mathrm{~mm}$ lens at $\mathrm{f} 1.0$, the diameter of the entrance pupil for the $50 \mathrm{~mm}$ lens will be twice that of the $25 \mathrm{~mm}$ lens, which will increase the light collection by a factor of 4 .

Experimental evaluation of the system will be performed along the lines presented in chapter V. In addition, a quantitative analysis of the contrast resolution will also be performed using appropriate contrast phantoms.

\subsection{Analysis of Noise Power Spectrum}

In the present $\mathrm{DQE}(\mathrm{f})$ analysis for the proposed system a white noise power spectrum (NPS) has been assumed. Therefore the analysis represents the upper limit of system performance that can be achieved. In order to get a more accurate estimate of the system response, the NPS of the proposed system will be determined using images acquired with this system. The NPS will be incorporated in the $\mathrm{DQE}(\mathrm{f})$ calculations to compute the $\mathrm{DQE}(\mathrm{f})$ more accurately. 


\subsection{Clinical Evaluation}

The clinical efficacy of the proposed portal imaging system will be determined by carrying out a subjective comparison of the images obtained using the CsI (Tl) - CCD system with those obtained using portal film. The comparison will be performed by a number of trained radiologists who will be asked to evaluate images from both the above modalities on a 5 point

scale. The responses will be analyzed using a rank test like the Wilcoxon sign-rank test and the intra-observer variability will be determined using kappa statistics. 


\section{CHAPTER 10}

\section{Conclusion}

The development of a high performance electronic portal imaging system based on a thick transparent x-ray scintillator coupled to a cooled CCD camera was discussed. A prototype system was developed using a $12.7 \mathrm{~cm}$ thick $\mathrm{CsI}(\mathrm{Tl})$ coupled to a liquid nitrogen cooled CCD camera. Theoretical and experimental results indicate that the transparent scintillator based EPID design shows good promise as a high performance portal imaging modality.

Amorphous silicon based flat panel EPIDs have become the focus of current EPID technology due to the fact that these flat panel systems practically eliminate the secondary (light) quantum sink. In contrast, TV camera based systems that use conventional powdered screens suffer from low light detection efficiency as well as low x-ray quantum detection efficiency (QDE). The use of a thick transparent scintillator increases the QDE by an order of magnitude compared to both, flat panel based EPIDs as well as conventional TV camera based EPIDs. The use of a highly sensitive modern CCD camera improves the light collection efficiency by an order of magnitude compared to conventional TV camera based systems. As a result of these increased x-ray and light quantum efficiencies, a TV camera based EPID using a thick transparent scintillator with a high quality CCD camera, presents a feasible alternative approach that can achieve performance levels better or at least equal to those of flat panel based EPIDs. 


\section{References}




\section{References}

1 P. Rubin and S.W. Dietmar, Principles of Radiation Oncology and Cancer Radiotherapy. In: Clinical Oncology, A Multidisciplinary Approach for Physicians and Students, ed. Rubin Philip. W. B. Saunders Company, 1992, pp. 71-90.

2 M.A. Henzler., A. Matloubieh, and H. Kubo, Basic Concepts of Radiation Physics. In: Clinical Oncology, A Multidisciplinary Approach for Physicians and Students, ed. Rubin Philip. W. B. Saunders Company, 1992, pp. 91-104.

3 R. Novario, P. Stucchi, L. Perna, and L. Conte, Radiotherapy treatment verification. Tumori.1998., vol. 84, pp. 144-149.

4 J.M. Balter, G.T. Chen, C.A. Pelizzari, S. Krishnasamy, S. Rubin, and S. Vijayakumar, Online repositioning during treatment of the prostate: a study of potential limits and gains. Int.J.Radiat.Oncol.Biol.Phys.1993., vol. 27, pp. 137-143.

5 A. Brahme, Dosimetric precision requirements in radiation therapy. Acta Radiol.Oncol.1984., vol. 23, pp. 379-391.

6 H.M. van, K.G. Gilhuijs, M.J. de, and A. Touw, Effect of image artifacts, organ motion, and poor segmentation on the reliability and accuracy of three-dimensional chamfer matching. Comput.Aided.Surg.1997., vol. 2, pp. 346-355.

7 H.M. van, A. Bruce, A.P. Kroes, T. Shouman, A. Touw, and J.V. Lebesque, Quantification of organ motion during conformal radiotherapy of the prostate by three dimensional image registration. Int.J.Radiat.Oncol.Biol.Phys.1995., vol. 33, pp. 1311-1320.

8 K.G. Gilhuijs and H.M. Van, Automatic on-line inspection of patient setup in radiation therapy using digital portal images. Med.Phys.1993., vol. 20, pp. 667-677.

9 H. Johns and J. Cunningham, The Physics of Radiology, Springfield, Illinois: Charles C. Thomas, 1983, pp. 167-216.

10 H. Johns H and J. Cunningham The Physics of Radiology, Springfield, Illinois: Charles C. Thomas, 1983, pp. 133-164.

11 H.I. Amols, L.E. Reinstein, and B. Lagueux, A quantitative assessment of portal film contrast as a function of beam energy. Med.Phys.1986., vol. 13, pp. 711-716.

12 D. Gur, M. Deutsch, C.R. Fuhrman, P.A. Clayton, J.C. Weiser, M.S. Rosenthal, and A.G. Bukovitz, The use of storage phosphors for portal imaging in radiation therapy: therapists' perception of image quality. Med.Phys.1989., vol. 16, pp. 132-136.

13 T. Falco and B.G. Fallone, Characteristics of metal-plate/film detectors at therapy energies. I. Modulation transfer function. Med.Phys.1998., vol. 25, pp. 2455-2462. 
14 P. Munro, J.A. Rawlinson, and A. Fenster, Therapy imaging: a signal-to-noise analysis of metal plate/film detectors. Med.Phys.1987., vol. 14, pp. 975-984.

15 A.G. Haus, R.E. Dickerson, K.E. Huff, S. Monte, B.A. Schlager, M. Atanas, and A. Matloubieh, Evaluation of a cassette-screen-film combination for radiation therapy portal localization imaging with improved contrast. Med.Phys.1997., vol. 24, pp. 1605-1608.

16 R. Sephton, M. Green, and C. Fitzpatrick, A new system for port films. Int.J.Radiat.Oncol.Biol.Phys.1989., vol. 16, pp. 251-258.

17 A.L. Boyer, L. Antonuk, A. Fenster, H.M. van, H. Meertens, P. Munro, L.E. Reinstein, and J. Wong, A review of electronic portal imaging devices (EPIDs). Med.Phys.1992., vol. pp. $1-16$.

18 H. Meertens, H.M. van, and J. Weeda, A liquid ionisation detector for digital radiography of therapeutic megavoltage photon beams. Phys.Med.Biol.1985., vol. 30, pp. 313-321.

19 H. Meertens, H.M. van, J. Bijhold, and H. Bartelink, First clinical experience with a newly developed electronic portal imaging device. Int.J.Radiat.Oncol.Biol.Phys.1990., vol. 18, pp. 1173-1181.

20 F.F. Yin, M.C. Schell, and P. Rubin, Input/output characteristics of a matrix ion-chamber electronic portal imaging device. Med.Phys.1994., vol. 21, pp. 1447-1454.

21 H.M. Van, J. Bijhold, B. Hoogervorst, and H. Meertens, Sampling methods for a matrix ionization chamber system. Med.Phys.1992., vol. pp. 409-418.

22 M. Essers, B.R. Hoogervorst, H.M. van, H. Lanson, and B.J. Mijnheer, Dosimetric characteristics of a liquid-filled electronic portal imaging device. Int.J.Radiat.Oncol.Biol.Phys.1995., vol. 33, pp. 1265-1272.

23 R. Boellaard, H.M. van, and B.J. Mijnheer, The dose response relationship of a liquidfilled electronic portal imaging device. Med.Phys.1996., vol. 23, pp. 1601-1611.

24 Y. Zhu, X.Q. Jiang, and D.J. Van, Portal dosimetry using a liquid ion chamber matrix: dose response studies. Med.Phys.1995., vol. 22, pp. 1101-1106.

25 M. Essers, R. Boellaard, H.M. van, H. Lanson, and B. Mijnheer, Transmission dosimetry with a liquid-filled electronic portal imaging device. Int.J.Radiat.Oncol.Biol.Phys.1996., vol. 34, pp. 931-941.

26 E.J. Morton, W. Swindell, D.G. Lewis, and P.M. Evans, A linear array, scintillation crystal-photodiode detector for megavoltage imaging. Med.Phys.1991., vol. 18, pp. 681691. 
27 L.E. Antonuk, J. Yorkston, W. Huang, H. Sandler, J.H. Siewerdsen, and Y. el-Mohri, Megavoltage imaging with a large-area, flat-panel, amorphous silicon imager. Int..Radiat.Oncol.Biol.Phys.1996., vol. 36, pp. 661-672.

28 L.E. Antonuk, J. Boudry, W. Huang, D.L. McShan, E.J. Morton, J. Yorkston, M.J. Longo, and R.A. Street, Demonstration of megavoltage and diagnostic x-ray imaging with hydrogenated amorphous silicon arrays [published erratum appears in Med Phys 1993 May-Jun;20(3):825] Med.Phys.1992., vol. 19, pp. 1455-1466.

29 P. Munro and D.C. Bouius, X-ray quantum limited portal imaging using amorphous silicon flat-panel arrays. Med.Phys.1998., vol. 25, pp. 689-702.

30 F.A. DiBianca, S. Samant, J. Laughter, J. Rasmussen, and C. Rodriguez, Use of a Kinestatic Charge Detector for Megavoltage Portal Imaging. Proc.SPIE, 3032, 1997, pp. 195-207.

31 B. Wowk, T. Radcliffe, K.W. Leszczynski, S. Shalev, and R. Rajapakshe, Optimization of metal/phosphor screens for on-line portal imaging. Med.Phys.1994., vol. 21, pp. 227-235.

32 T. Radcliffe, G. Barnea, B. Wowk, R. Rajapakshe, and S. Shalev, Monte Carlo optimization of metal/phosphor screens at megavoltage energies. Med.Phys.1993., vol. 20, pp. 1161-1169.

33 F.H Attix. Introduction to Radiological Physics and Radiation Dosimetry, John Wiley \& Sons Inc., 1986.

34 G.F. Knoll Radiation Detection and Measurement, John Wiley \& Sons, 1989.

35 C.L. Woody, J.A. Kierstead et al, Radiation Damage in Undoped CsI and CsI(Tl) IEEE Trans.on Nuclear Science, 1992, vol. 39, pp. 524-531.

36 Beynon J.D.E. and Lamb D.R. Charge-coupled devices and their applications, McGRAW-HILL Book Company (UK) Limited, 1989.

37 D.M. Kambeyanda, Determination of spatial resolution and temporal rate of bone demineralization using digital radiographic techniques. University of Tennessee, Memphis. M.S. thesis, 1995, pp. 69-89.

38 M.G. Herman, R.A. Abrams, and R.R. Mayer, Clinical use of on-line portal imaging for daily patient treatment verification [see comments] Int.J.Radiat.Oncol.Biol.Phys.1994., vol. 28, pp. 1017-1023,

39 R.M. Nishikawa, M.J. Yaffe, and R.B. Holmes, Effect of finite phosphor thickness on detective quantum efficiency. Med.Phys.1989., vol. 16, pp. 773-780,

40 H.D. Zeman, S. Samant, and J. Rasmussen, $6 \mathrm{MVp}$ x-ray imaging with a transparent scintillator x-ray detector Proc SPIE 3032, 1997, pp. 388-394. 
41 H.D. Zeman, S. Samant, G. Lovhoiden, B. Weinberg, and A. Sawant, Portal imaging with a CsI (Tl) transparent scintillator x-ray detector Proc.SPIE, 3336, 1998, pp. 175-187.

42 Zeman H.D., Samant S., and Sawant A., Human subject portal images with a CsI (Tl) transparent scintillator Proc.SPIE, 3659-71, 1999, vol. pp. 682-693.

43 PixelVision Inc., Tigard OR 97223. PlutoCCD Series, Specifications Sheet., 1998.

44 D.A. Jaffray, K. Chawla, C. Yu, and J.W. Wong, Dual-beam imaging for online verification of radiotherapy field placement. Int.J.Radiat.Oncol.Biol.Phys.1995., vol. 33, pp. 1273-1280.

45 J.C. Dainty and R. Shaw Image Science, Academic Press Inc. (London) Ltd., 1974.

46 J. Halbleib, Structure and operation of the ITS code system. In: Monte Carlo Transport of Electrons and Photons, eds. Jenkins T.M., Nelson W.R., and Rindi A. Plenum Press, 1988, pp. 249-262.

47 J. Halbleib, Applications of the ITS codes. In: Monte Carlo Transport of Electrons and Photons, eds. Jenkins T.M., Nelson W.R., and Rindi A. Plenum Press, 1988, pp. 263-286.

48 R. Mohan, C. Chui, and L. Lidofsky, Energy and angular distributions of photons from medical linear accelerators. Med.Phys.1985., vol. 12, pp. 592-597.

49 W. Swindell, The lens coupling efficiency in megavoltage imaging. Med.Phys.1991., vol. 18, pp. 1152-1153.

50 J.A. Sorenson and M.E. Phelps, Physics in Nuclear Medicine, W.B. Saunders Co., 1987, Table 4-2, p.77.

51 E. Gramsch, K.G. Lynn et al, Silicon PIN photodetectors in high resolution nuclear spectroscopy Nucl.Instr.and Meth.in Phys.Res., 1992, vol. A311, pp. 529-538.

52 M. Lachaine and B.G. Fallone, Monte Carlo detective quantum efficiency and scatter studies of a metal/a-Se portal detector. Med.Phys.1998 Jul., vol. 25, pp. 1186-1194.

53 J.P. Bissonnette, I.A. Cunningham, and P. Munro, Optimal phosphor thickness for portal imaging. Med.Phys.1997., vol. 24, pp. 803-814.

54 D. DeJager and R. Kingslake, Photographic Optics. In: SPSE Handbook of Photographic Science and Engineering, ed. Thomas W.Jr. John Wiley \& Sons, 1973.pp. 159-255.

55 Astromed Ltd., CCD Data Sheet and Selection Guide 1992. Microphotonics, New York.

56 B.J. Heijmen, K.L. Pasma, M. Kroonwijk, V.G. Althof, B.J. de, A.G. Visser, and H. Huizenga, Portal dose measurement in radiotherapy using an electronic portal imaging device (EPID). Phys.Med.Biol.1995., vol. 40, pp. 1943-1955, 
57 P. Munro, D.C. Bouius, Moseley J., Martin L.C., Zhang Y., and D.A. Jaffray. Glaring errors in transit dosimetry., Submitted to Med. Phys.

58 R.C. Gonzalez and E.R. Woods, Digital Image Processing, Addison-Wesley Publishing Company, 1992. pp. 166-185.

59 J.C. Weiser, D. Gur, R.C. Gennari, and M. Deutsch, Evaluation of analog contrast enhancement and digital unsharp masking in low-contrast portal images. Med.Phys.1990., vol. 17, pp. 122-125.

$60 \mathrm{~N}$. Gallagher and G. Wise, A theoretical analysis of the properties of median filters IEEE Trans.Acoustics, Speech and Signal Processing, Jan 12, 1981, vol. ASSP-29, pp. 11361141.

61 G. Arce and R. Foster, Detail preserving rank-order based filters for image processing IEEE Trans.Acoustics, Speech and Signal Processing, Jan 1, 1989, vol. 37-1, pp. 83-98.

62 L. Yin et al, Weighted median filters: A tutorial IEEE Trans.On Circuits and Systems, Jan 3, 1996, vol. 43-3, pp. 157-192.

63 J. Tuckey, Exploratory Data Analysis, Reading MA: Addison Wesley, 1977.

64 H.D. Zeman, The Processing of Intravenous Coronary Angiograms Produced by Synchrotron Radiation. In: Handbook of Synchrotron Radiation, Anonymous Elsevier Science Publishers B.V., 1991, pp. 697-718.

65 H.D. Zeman, F. A. DiBianca, and G. Lovhoiden, High resolution x-ray imaging with a Gd203(Eu) transparent ceramic scintillator Proc SPIE [2432-44], 1995, pp. 454-461.

66 F.A. DiBianca, V. Gupta et al, Ultrahigh resolution CT and DR scanner Proc SPIE [365905], 1999, pp. 56-64.

67 A.K. Jain, Fundamentals of Digital Image Processing, Prentice-Hall, 1989.

$68 \mathrm{X}$. Zhong et al, Speckle reduction and contrast enhancement of echocardiograms via multiscale nonlinear processing, IEEE Trans.Medical Imaging, 1998, vol. 17-4.

69 M.J. Lester et al, Local transforms for biomedical image analysis Comput.Graphics, Image Processing, 1980, vol. 13, pp. 17-30.

70 D. M. Muratore, B. M. Dawant, and R. L. Galloway, Vertebral surface extraction from ultrasound images for technology-guided therapy Proc SPIE [3661-160], 1999. 


\section{VITA}

Amit Sawant received his undergraduate degree in Biomedical Engineering from the University of Bombay in 1996. He entered the University of Tennessee, Memphis and University of Memphis, Tennessee, Joint Program in Biomedical Engineering in August 1997, majoring in Medical Imaging.

He is presently employed as a Software Engineer in the Radiation Oncology Department at St. Jude Children's Research Hospital, Memphis. 